\title{
The effects of interactive video (DDR) on heart rate, perceived exertion, step count, self -efficacy, and enjoyment in elementary school children
}

\author{
Barbara J. Olmsted \\ West Virginia University
}

Follow this and additional works at: https://researchrepository.wvu.edu/etd

\section{Recommended Citation}

Olmsted, Barbara J., "The effects of interactive video (DDR) on heart rate, perceived exertion, step count, self -efficacy, and enjoyment in elementary school children" (2006). Graduate Theses, Dissertations, and Problem Reports. 4253.

https://researchrepository.wvu.edu/etd/4253

This Dissertation is protected by copyright and/or related rights. It has been brought to you by the The Research Repository @ WVU with permission from the rights-holder(s). You are free to use this Dissertation in any way that is permitted by the copyright and related rights legislation that applies to your use. For other uses you must obtain permission from the rights-holder(s) directly, unless additional rights are indicated by a Creative Commons license in the record and/ or on the work itself. This Dissertation has been accepted for inclusion in WVU Graduate Theses, Dissertations, and Problem Reports collection by an authorized administrator of The Research Repository @ WVU.

For more information, please contact researchrepository@mail.wvu.edu. 
The Effects of Interactive Video (DDR) on Heart Rate, Perceived Exertion, Step

Count, Self-Efficacy, and Enjoyment in Elementary School Children

\author{
Barbara J. Olmsted
}

\author{
Dissertation submitted to the \\ School of Physical Education \\ at West Virginia University \\ in partial fulfillment of the requirements \\ for the degree of \\ Doctor of Education \\ in \\ Physical Education Teacher Education
}
Andrew H. Hawkins, Ph.D., Chair
Linda M. Carson, Ed.D.
Lynn D. Housner, Ph.D.
William E. Reger-Nash, Ed.D.
Richard T. Walls, Ph.D.

School of Physical Education

Morgantown, WV

2006

Keywords: physical activity, perceived exertion, step count, self-efficacy, enjoyment, active time, interactive video

Copyright 2006 Barbara Olmsted 


\begin{abstract}
The Effects of Interactive Video (DDR) on Heart Rate, Perceived Exertion, Step Count, Self-Efficacy, and Enjoyment in Elementary School Children
\end{abstract}

Barbara J. Olmsted

Rising levels of obesity continue to challenge physical educators to engage their students in activities sufficiently vigorous to accrue health-related benefits. Physically active video games are now recognized as a way to enhance physical activity levels in children. A school-based intervention in fifth- and sixth-grade participants $(\mathrm{N}=33)$ compared six lessons each of Dance Dance Revolution (DDR), volleyball, and an exercise video (TaeBo) according to the following dependent variables: heart rate, step count, active time, rate of perceived exertion, enjoyment, and self-efficacy. Repeated measures withinsubjects comparisons revealed significant main effects for activity type across five dependent variables, and significant interactions for four dependent variables $(p<.05)$. DDR elicited the highest heart rates, step counts, and enjoyment scores. Perceived exertion rates indicated that participants exercised vigorously during DDR without recognizing levels of fatigue. Self-efficacy did not change significantly. DDR is a viable, non-traditional way to increase physical activity in elementary schools. 


\section{Acknowledgements}

A project of this magnitude is not possible without the assistance of many important individuals. Each person mentioned herein has contributed in a unique way to guide me along this journey; a journey which has introduced me to some special people in West Virginia, and one which has confirmed the support of those at home.

I would first like to acknowledge the members of my committee, whom, under the capable leadership of Dr. Andrew Hawkins showed no hesitation in supporting my research from afar. Dr. Hawkins, thank you for your patience, your willingness to review my document under tight timelines, and your helpful suggestions during all stages of preparation. To Dr. Richard Walls, thank you for your unwavering commitment to appropriate statistical conventions and editorial detail. I am confident that because of your interest, I have been able to prepare a professional document. To Dr. Linda Carson, thank you for your enthusiasm, your creative ideas, and your friendship. I have you to credit for recognizing the potential of applying DDR to an educational setting.

I would be remiss without recognizing the time and expertise of Dr. Andrew Weeks at Nipissing University. His knowledge of statistical applications in SPSS kept me from drowning in numbers. Thank you also to Mr. Brent Pigott, whose data recording assistance was much appreciated.

I would like to acknowledge Mr. Colin Vickers, Director of the Near North Board of Education, who, in seeing DDR for the first time was convinced of its utility in engaging students in physical activity and quickly consented to my research proposal in one of his elementary schools. Additionally, I would like to sincerely thank Mr. Scott Dunn (Principal) and teachers Ms. Sandy Chowns and Ms. Kim Rickertsen for their 
interest, patience, understanding, and commitment in allowing this research to be conducted in their school. In particular, the $5^{\text {th }}$ and $6^{\text {th }}$ grade students at J.W. Trusler School are shining examples of eager, enthusiastic learners under any circumstance!

On a personal level, I have been fortunate to have parents who continue to support me no matter the endeavor. This became particularly poignant since I decided to become a student once again after so many years. As always, my parents have been sounding boards, guidance counselors, providers, and most importantly, there for me.

Finally, to my partner Heather, thank you for supporting me every step of the way. Despite the time away, the distance, the phone calls, and the traveling, it was your understanding nature and confidence in me that kept me motivated to complete this journey. I look forward to the next one! 
List of Tables............................................................

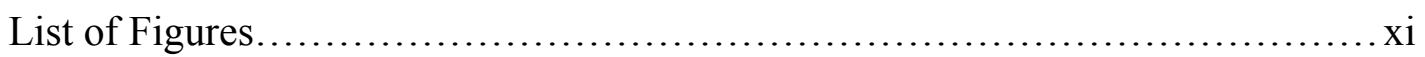

Chapter One - Introduction and Statement of Problem....................... 1

Introduction...................................................... 1

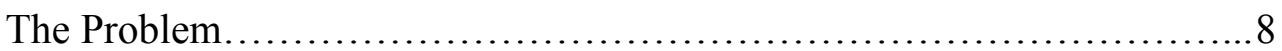

Statement of problem.................................... 8

Scope of study........................................ 9

Basic Assumptions....................................... 11

Limitations................................................ 11

Definition of terms..................................... 12

Significance of the Study....................................... 13

Chapter Two - Selected Review of Literature.............................. 16

Introduction................................................... 16

Physical Activity Patterns of Children............................... 16

Heart Rate as a Measure of Physical Activity Intensity........... 18

Pedometry as a Measure of Physical Activity Intensity.............. 23

Rate of Perceived Exertion as a Measure of

Physical Activity Intensity .............................. 28

Psychological Factors Influencing Physical Activity Behavior............. 30

Enjoyment of Physical Activity............................... 32

Self-Efficacy for Physical Activity ............................ 34 
Physical Activity in Physical Education.............................. 36

Video Games...................................................40

Video Game Use........................................ 40

Physically Active Video Games............................. 43

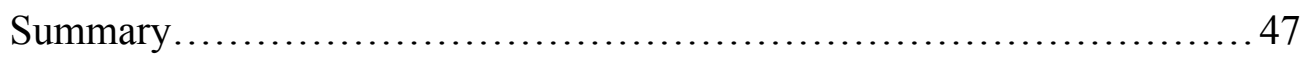

Chapter Three - Method................................................ 49

Participants.................................................49

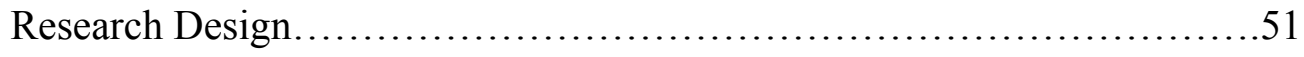

Instrumentation.................................................... 56

Heart Rate............................................... 57

Step Count and Active Time............................ 57

Rate of Perceived Exertion................................ 58

Enjoyment.............................................59

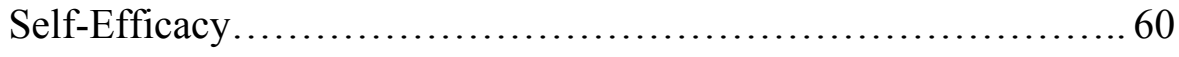

Procedure...................................................... 61

Heart Rate Monitors and Pedometers......................... 63

Questionnaires and Scales................................6 65

DDR Sessions............................................. 66

Tae-Bo Sessions.......................................67

Volleyball Sessions.......................................6 68

Research Hypotheses............................................ 69

Data Analyses............................................... 69

Chapter Four - Results............................................. 74 
Demographic Data.............................................. 74

Heart Rate.................................................... 78

Step Count........................................................ 82

Active Time....................................................... 85

Perceived Exertion...................................................... 89

Heart Rate and Perceived Exertion................................... 92

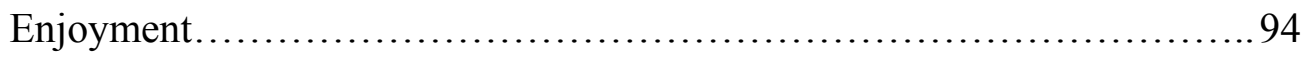

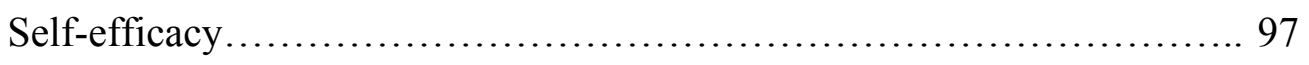

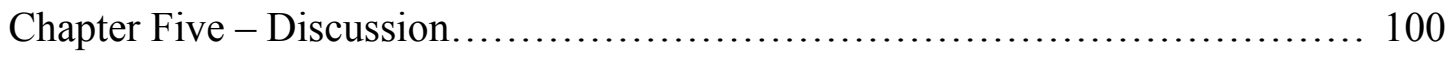

Demographics ................................................... 100

Heart Rate.......................................................... 103

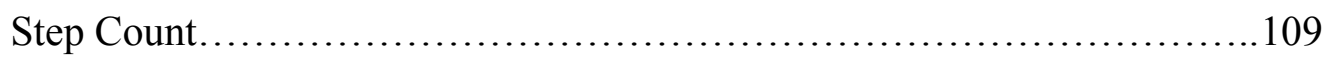

Active Time ........................................................... 112

Perceived Exertion................................................. 114

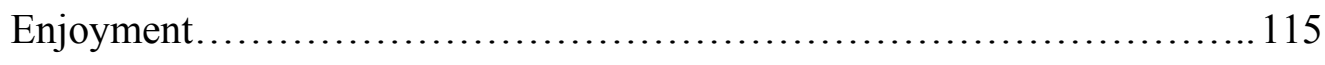

Self-efficacy .......................................................... 122

Limitations......................................................... 124

Future Directions.................................................. 131

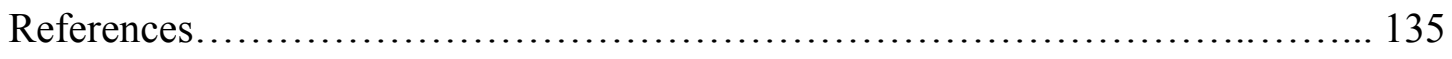

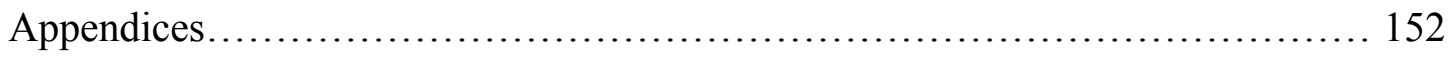

A. Letter of Permission to Conduct Research......................... 152

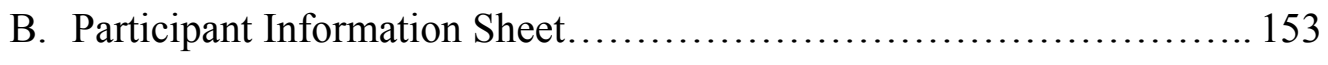

C. West Virginia University IRB Approval........................... 154 
D. Nipissing University Ethics Approval.............................. 155

E. Student Assent Form.............................................. 156

F. Parent Consent Form........................................... 157

G. Teacher Lesson Plans............................................... 161

H. Permission for Use of OMNI Perceived Exertion Scale..................174

I. OMNI Scale of Perceived Exertion................................ 176

J. Permission for Use of Self-Efficacy Scale and Enjoyment Scale........177

K. Self-Efficacy Scale .............................................. 179

L. Physical Activity Enjoyment Scale.............................. 180

M. Letter of Permission for Youth Physical Activity Promotion Model..... 181

N. Mean Scores per Trial for Volleyball, Tae-Bo, and DDR............... 182

O. Mean Scores According to Group (Gender, Grade Level, BMI)......... 186

P. Pearson Product-Moment Correlation Coefficients Among

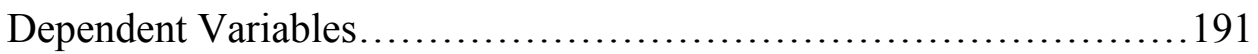




\section{List of Tables}

Table 1. Correlations ( $r$ ) of Two Heart Rate Monitors Compared to an Electrocardiogram Recording............................... 21

Table 2. Correlation Between Pedometer Step Count and Physical Activity Parameters................................................. 26

Table 3. Mean Age, height, Weight and Body Mass Index ( \pm Standard Deviation of Fifth- and Sixth-Grade Participants.................. 50

Table 4. Random Orders of Activity Type Within Blocks....................... 52

Table 5. Independent and Dependent Variables............................. 54

Table 6. Sample 3 x 6 Repeated Measures Analysis of Variance for Heart Rate ....70

Table 7. Sample Split Plot Factorial 222.3 Repeated Measures ANOVA for

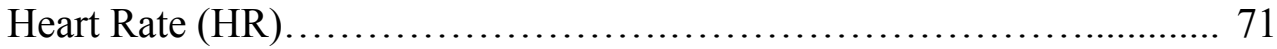

Table 8. Pre- and Post-intervention Descriptive data in Fifth- and Sixth-Grade Participants................................................... 75

Table 9. Body Mass Index (BMI) Frequencies in Fifth- and Sixth-Grade

Participants................................................. 76

Table 10. Frequency Distribution of Physical Activity on Weekdays and Weekends in Fifth- and Sixth-Grade Participants................... 77

Table 11. Frequency Distribution of DDR Experience in Fifth- and Sixth-Grade Participants.............................................. 78

Table 12. Multiple Comparisons for the Significant Main Effect of Activity Type on Heart Rate. 80 
Table 13. Multiple Comparisons for the Significant Main Effect of Activity Type on Step Count........................................ 84

Table 14. Multiple Comparisons for the Significant Main Effect of Activity Type on Percent Active Time................................. 88

Table 15. Multiple Comparisons for the Significant Main Effect of Activity Type on Rate of Perceived Exertion. 91

Table 16. Multiple Comparisons for the Significant Main Effect of Activity Type on Enjoyment Score....................................96

Table 17. Mean Self-efficacy Scores and Subsequent $t$ test Results for Fifth- and Sixth-Grade Participants.................................. 98

Table 18. Percent of Responses to Self-efficacy Questionnaire Items Post-intervention..................................................... 99 


\section{List of Figures}

Figure 1. Photograph of the Cateye PL-6000 earlobe heart rate monitor.......... 20

Figure 2. Photograph of the Walk4Life LS 2505 pedometer.................. 27

Figure 3. A conceptual diagram of the youth physical activity promotion model... 31

Figure 4. Mean heart rate per trial for all participants......................... 79

Figure 5. Mean heart rate (+standard deviation) for all participants in volleyball, Tae-Bo, and DDR........................................... 80

Figure 6. Mean heart rate (+standard deviation) for two BMI groups for volleyball, Tae-Bo, and DDR................................ 82

Figure 7. Mean step count per trial for all participants......................... 83

Figure 8. Mean step count (+standard deviation) for each activity type for all participants

Figure 9. Percent active time per trial for all participants in volleyball, Tae-Bo, and DDR..................................................... 87

Figure 10. Percent active time (+standard deviation) for each activity type........ 88

Figure 11. Mean rate of perceived exertion per trial for all participants............ 90

Figure 12. Mean rate of perceived exertion for volleyball, Tae-Bo, and DDR..... 91

Figure 13. Scatter diagram of mean scores per trial for heart rate versus rate of perceived exertion........................................93

Figure 14. Mean heart rate and mean RPE for volleyball, Tae-Bo, and DDR...... 94

Figure 15. Mean enjoyment score per trial for all participants................. 95

Figure 16. Mean enjoyment score for volleyball, Tae-Bo, and DDR..............96 


\section{CHAPTER 1}

\section{INTRODUCTION AND STATEMENT OF THE PROBLEM}

\section{Introduction}

Of ever increasing concern to physical educators is the number of overweight and obese students in schools. Nearly one third of children aged 6 through 19 are either overweight or at risk of overweight (Hedley et al., 2004; USDHHS, 1996). This problem, now approaching epidemic proportions, is directly related to levels of physical inactivity (Dietz, 2004; Tremblay \& Willms, 2003; Trost, Kerr, Ward, \& Pate., 2001). Lifestyle changes in recent years have seen children spending more leisure time watching television or playing video games (Faith et al., 2001, Gortmaker et al., 1990). As a result, time spent at school (including recess, physical education classes, and extracurricular activities) may be the only opportunity presented to children to engage in physical activity. The fact that youth today have become so accustomed to using technological devices, such as computers and video games, might actually prove to be an asset for school-based interventions designed to slow or reverse the current trend of physical inactivity. The use of technology in physical education classes or during non-academic school hours may contribute to greater student participation, and therefore, enhanced health-related fitness benefits.

Physical education teachers continue to try new approaches and strategies to engage their students in appropriate activities designed to enhance not only their current state of fitness, but also their commitment to healthy, active lifestyles. This challenge remains particularly critical given the marginal status often assigned to school physical education programs and the emphasis on basic academic skills and standardized testing. 
Providing structured activities that increase heart rates sufficiently to impact healthrelated fitness should be one major goal for teachers. Indeed, simply increasing physical activity levels above resting states for sustained periods of time would positively impact health status in most children (Sallis \& McKenzie, 1991; USDHHS, 1996). At the present time, most children's activities are not sufficiently intense or lengthy enough to accrue health-related benefits (Hoos, Gerver, Kester, \& Westerterp, 2003; Steinbeck, 2001). Video games often represent a child's first exposure to computers, and such games have been criticized for their adverse effects (Anderson, 2004; Emes, 1997; Rideout, Vandewater \& Wartella, 2003). Yet, video games still represent a large part of a child's leisure time. As reported in a study by the Kaiser Foundation (Anand \& Krosnick, 2005), the average two-year-old child will sit in front of a television, computer, or video game for an average of two hours per day. This astonishing finding must be closely examined for its potential to physically engage children using the video game platform. Vandewater, Shim and Caplovitz (2004) have found that video game use and weight status were related in children 1-12 years of age. Children aged 9-12 with higher weight status spent a significant amount of time playing video games, and this finding, according to the authors, plays a significant role in the complex issue of childhood obesity in the United States. Kautianen, Koivusilta, Lintonen, Virtanen, and Rimpela (2005) more recently reported that playing digital games (video, computer and console games) was not related to overweight in teens aged 14-18 years, yet television viewing was positively associated with overweight in this age group. Physiologic responses to video game play involve increases in metabolic rate and cardiovascular stimulation, similar to mildintensity exercise (Segal \& Dietz, 1991). While not significant to impact health-related 
fitness, further research is necessary to determine this relationship. This is particularly true for the latest health-based video game technology involving large body movements.

The prevalence of health-oriented video games has increased in recent years. Some of these games have replaced the handheld controller with a touch pad or similar device requiring significant body movements to follow cues on the screen. Dance Dance Revolution (DDR), created by Konami Digital Entertainment in 1998, is one such game that includes a three-foot square dance pad (placed on the floor) with electronic sensors to record foot touches, and the player responds to auditory (musical) and visual cues on the television screen. Successful performance, and thus, a high score, is dependent upon the ability of the performer to make rhythmically accurate responses to the appearance of directional arrows moving up the screen. A final grade and total score are recorded at the end of each song, providing immediate feedback to the performer. Beginners must proceed with fairly slow songs to become accustomed to the format of the game and to develop footwork patterns. As a result, exercise heart rates may not be elevated significantly above resting levels. Once participants develop some competence with the game, they progress to higher tempos and more complex foot patterns, thus increasing the likelihood that heart rates will increase sufficiently to impact fitness.

While there is a dearth of research involving this emerging form of physically active engagement, Ridley and Olds (2001) have found that energy expenditures of 1112-year-old children while playing these types of interactive video formats far exceed that of games using a handheld controller. These authors reported energy expenditures were equivalent to walking or jogging at speeds of 3 to 7.5 kilometers per hour. Tan, Aziz, Chua and Teh (2002) suggest that Dance Dance Revolution can meet minimum 
guidelines for exercise intensity as recommended by the American College of Sports Medicine (ACSM).

DDR was originally designed purely for entertainment, but some researchers are beginning to recognize the potential value in this appealing game as a means to benefit children with learning difficulties. McGraw, Burdette, Seale, and Gregg (2002) have found that DDR used in the school setting enhanced reading performance and attention in middle school children with ADHD (Attention Deficit Hyperactive Disorder). These authors concluded that ADHD affected executive functioning and motor control but DDR enhanced the neural networks involved in reading and attention.

The growing popularity of this video game format provides an excellent opportunity to explore the factors determining participation as well as the potential to integrate this type of technology into educational settings. DDR may be used in a number of ways, namely during physical education classes, recess periods, and other nonacademic times throughout the school day. This may be particularly attractive for schools lacking regular gym availability for all classes, since DDR does not require a proper gym floor or a large space for many students to be active at one time. Because DDR requires multiple units for large group participation, issues such as supervision, cost, storage, and security might be concerns. These obstacles may be overcome, however, if DDR is deemed to be a valid tool to enhance physical activity participation.

Physical activity determinants in youth are multifactorial, so a single causal factor for subsequent participation is difficult to identify (Trost et al., 1997). A number of theoretical models have attempted to describe the determinants of physical activity behaviors in adults, but none has been able to embrace the unique developmental 
characteristics of children (Council for Physical Education for Children, 1998). Welk (1999) has proposed a youth physical activity promotion model that synthesizes a number of theories to describe the social, environmental and personal factors affecting physical activity participation. As a result, this model takes a broad perspective in examining physical activity determinants. Self-efficacy and enjoyment are identified by Welk as two key predisposing factors predictive of subsequent physical activity engagement. Selfefficacy is a domain specific concept relating to one's own belief in the ability to perform a particular activity (Bandura, 1997; Chase, 1998). For example, an individual may have a high self-efficacy for basketball and a low self-efficacy for volleyball. According to Bandura's (1977) social-cognitive theory, this person is more likely to engage in basketball because of greater perceived competence in that particular sport. Ryan and Dzewaltowksi (2002) reported that involvement in physical activity among sixth and seventh grade students is related to one's perceived ability to find and create supportive environments. Winters, Petosa, and Charlton (2003) suggested that educators should focus on psychosocial variables such as goal-setting, self-monitoring, social support and self-efficacy in enhancing physical activity participation in educational settings. Since self-efficacy is predictive of physical activity participation (Allison, Dwyer, \& Makin, 1999), efforts to enhance self-efficacy may subsequently have a positive impact on physically active lifestyles. According to Bandura (1997), self-efficacy derives largely from mastery experiences. An individual who successfully executes a task will believe that he or she has the capabilities necessary to engage in that task on subsequent occasions. Developing competence in DDR may play a role in physical activity selfefficacy. 
Enjoyment is a positive affective state reflecting feelings of pleasure and fun (Berger, 2004). The enjoyment of physical activity has received recent attention in the literature as researchers strive to determine the underlying factors affecting exercise participation. As levels of physical activity in children have steadily declined, physical educators, health promotion practitioners, parents and many others have recognized that if children do not enjoy what they are doing, the likelihood that they will continue is greatly reduced. Prochaska, Sallis, Slymen, and McKenzie (2003) found that levels of enjoyment of physical education class steadily decreased from the beginning of fourth grade to the end of the sixth grade. These findings suggest that physical educators need to re-examine curriculum offerings so that they can provide relevant, engaging activities that will maintain interest through the middle school grades and beyond.

Enjoyment has been measured using a number of different scales, and one of the most frequently used instruments is the Physical Activity Enjoyment Scale (PACES) developed by Kendzierski and DeCarlo (1991). This 18-item inventory has been established as a reliable and valid measure of physical activity enjoyment in children and adolescents (Crocker, Bouffard, \& Gessaroli, 1995; Kenzierski \& DeCarlo, 1991; Motl et al., 2001). Dishman et al. (2005) have modified this scale to assess enjoyment in middle school children. These researchers established a 16-point scale to better represent the comprehension ability of this age group, and established factorial and construct validity in a study of eighth-grade girls. These researchers confirmed the validity of their instrument using another group of sixth- and eighth-grade girls (Dishman et al., in press). As indicated by Kimiecik and Harris (1996), enjoyment is a process, not a product of physical activity engagement. As such, individuals will engage in an enjoyable activity 
for the experience and the positive feelings it evokes. In school settings, enjoyment of physical education can be a powerful mood enhancer, and therefore, positively impact the learning environment (Berger, 2004). Additionally, the enjoyment of physical education may increase adherence to a physically active lifestyle.

The rating of perceived exertion has become a popular instrument to measure perceptions of exercise intensity because of its ease of use and understanding (Noble \& Noble, 1998). The sensory cues produced by the body (e.g. increased ventilation, increased heart rate, sweating, muscle strain or fatigue) are interpreted differently by each individual to produce personal feelings of effort. Although a subjective measure, many researchers have established the linearity between heart rate and perceived exertion, thus making perceived exertion a valid and reliable measure of exercise intensity (Hampson, Gibson, Lambert \& Noakes, 2001). Validity of perceived exertion measures has also been established in children (Mahon, Gay, \& Stolen, 1998). Robertson (2004) has conducted extensive research using his self-developed perceived exertion instrument with children performing a variety of exercise modalities which include cycling, walking, running, and stepping. One important quality of this instrument is the addition of pictures showing the same individual (child) experiencing increasing levels of exertion. Pictures are matched to a numerical rating scale. This permits young children to make appropriate associations between the pictures and their perceived level of effort (Utter, Robertson, Nieman \& Kang, 2002). It would be interesting to examine how perceived exertion may be affected when attentional focus is taken away from bodily sensations. The fact that DDR requires the participant to follow visual and auditory cues might suggest that DDR could mask 
these sensations, and participants may think they are working less intensely than they actually are. Heart rate measures will help to determine this relationship.

The understanding of physical activity engagement in youth continues to challenge those who recognize the value of adopting healthy lifestyle behaviors at an early age. It is anticipated that this knowledge will guide physical educators in planning and implementing relevant, quality programming in schools. Several research questions emerge from reviewing the literature:

1. Does DDR sufficiently increase heart rate to accrue health benefits in elementary school children?

2. What is the effect of DDR on physical activity self-efficacy, enjoyment, and perceived exertion in elementary school children?

3. How do DDR class sessions compare to other physical education classes in terms of heart rate, step counts, activity time, perceived exertion, self-efficacy and enjoyment?

The Problem

\section{Statement of the Problem}

The purpose of this study was to investigate the impact of Dance Dance Revolution on elementary school children with respect to average heart rate, step count, activity time, perceived exertion, physical activity self-efficacy, and physical activity enjoyment. In comparing DDR with two other physical education activities (volleyball and Tae-Bo, an exercise video), the significance of these effects would be elucidated. 


\section{Scope of the Study}

A convenience sample of grades five and six students was recruited from one school in the Near North District Public School Board in North Bay, Ontario, Canada. A total of two classes $(N=47)$ was included in this study; one sixth-grade class and one fifth-grade class. Institutional Review for the Protection of Human Subjects (IRB) approval from West Virginia University and Ethics Committee approval from Nipissing University was secured prior to the collection of data. In addition, parental consent was obtained for all participants, as required by Nipissing University Ethics guidelines. Each participant also signed an assent form to acknowledge their agreement to participate. Demographic data, including height, weight, age, gender, experience with DDR, and current level of physical activity were collected. Study design was a one-way within subjects repeated measures format in which all participants were subjected to each of three treatments (DDR, Tae-Bo, and regular physical education), repeated randomly so that each treatment was imposed six times over the course of 18 consecutive physical education classes. The planned activity for regular physical education classes at this time of the year was volleyball. DDR, Tae-Bo, and volleyball served as the three levels of the independent variable. Because the participants received physical education class three times per week, the total length of the intervention was six weeks (January 26-March 10, 2006). Prior to data collection, all participants were given the opportunity to take part in two familiarization sessions each of DDR and Tae-Bo. Participants self selected songs for DDR play, and continued for the duration of the class. The exercise video lessons involved a commercially available video called Tae-Bo, which is a style of exercise involving boxing movements of arms and legs. Participants simply followed the visual 
and auditory cues of the instructor on the video. Physical education classes involved a series of six volleyball lessons which were part of the regular grade five and six curriculum. Grade-appropriate lessons were presented in a sequential fashion, designed to increase skill development and game play. Teachers used their own lesson plans for volleyball classes. All lessons were presented and supervised by the classroom teachers assigned to each grade. Lesson plans were provided for DDR and Tae-Bo to maintain consistency during implementation. Sufficient DDR units and dance pads were placed within the school so that all participants engaged in DDR at the same time. Each DDR unit could accommodate four dance pads, so groups of four students worked together on the same songs. All lessons took place in the gymnasium.

Each lesson was 30 minutes in length. Questionnaires for perceived exertion and enjoyment were completed in the classroom immediately following each lesson. Lessons took place during regularly scheduled physical education classes, so students received an average of three lessons per week (e.g. DDR, Tae-Bo, and volleyball). Measures of all dependent variables were taken using the following instruments: heart rate monitors, pedometers, rate of perceived exertion scale, enjoyment scale, and self-efficacy scale. Average heart rate was measured during each physical activity lesson using the Cateye heart rate monitor (Cateye Co., Ltd.) attached to the ear lobe of each participant. The Cateye heart rate monitor also recorded total time for each lesson. Pedometers (Walk4Life, Inc.) measured total step counts and total active time for each session. Rate of perceived exertion was measured following each activity session using the OMNI Picture System of Perceived Exertion (Robertson, 2004). Self-efficacy was assessed using the physical activity self-efficacy instrument (questionnaire) developed by 
Dishman and his colleagues (Dishman et al., in press). Self-efficacy was measured before the intervention, and once again at the completion of the intervention period. The Physical Activity Enjoyment Scale developed by Dishman et al. (2005) was used to establish levels of enjoyment over the course of the intervention. Participants completed both the OMNI scale and the enjoyment questionnaire immediately following each lesson. Data gathered were subjected to a repeated measures analysis of variance. This determined main effects and interactions among variables.

\section{Basic Assumptions}

The basic assumptions for this study included:

1. Six sessions of each treatment would be sufficient to detect differences among independent and dependent variables over time.

2. Minimal absenteeism would not adversely affect data analysis.

\section{Limitations of the Study}

There were a number of limitations that may have affected the validity of this study. The sample was one of convenience involving only one school, so generalizability may not be readily apparent. There was a wide variation in athletic ability, level of fitness and self-efficacy among students, and this may have affected results. While this was to be expected in any physical education class, each student received all treatments, so within subject comparisons should have minimized this concern. Some participants may have had DDR at home and could have been avid players. Experienced players are more competent, and therefore can choose more difficult songs. This would require more vigorous movements and thus higher heart rates. Because all participants underwent a familiarization period for all three treatments and instrumentation procedures for the 
heart rate monitors and pedometers, this limitation was not expected to affect results significantly.

The fact that a video game was being used in an educational setting may have created some concern with teachers, parents, and administrators. For example, parents may not have permitted their son or daughter to be exposed to this video game format in an educational setting. Additionally, some teachers may not have been in favor of a video game program in their school setting. It was not anticipated that these issues would negatively impact the implementation of this study. Through exposure to DDR at preliminary information meetings, parents were provided an opportunity to try both DDR and Tae-Bo. Teachers were given the opportunity to use DDR at home for a period of time to become familiar with the game. Both teachers involved were very supportive and excited about adding DDR to their physical education repertoire.

Six Xbox units were in use simultaneously, which created a noisy environment. This could have interfered with student learning in nearby areas of the school, or it could have affected participant engagement in the study. This was addressed by regulating volume levels on each video monitor, and examining the physical arrangement of each video game unit. Television monitors were placed in a circle in the middle of the gymnasium with the screens facing outward. This maximized space for DDR pads and minimized the distraction from one monitor to the next.

\section{Definition of Terms}

Active Time - Total movement time during a physical education class, measured in this study by the Walk4Life pedometer. 
Enjoyment - "An optimal psychological state that leads to performing an activity primarily for its own sake and is associated with positive feeling states." (Kimiecik \& Harris, 1996, p. 256).

Heart Rate - Number of beats of the heart per minute (bpm), measured either directly over the heart or at a location where the pulse is felt.

Perceived Exertion - Feelings of discomfort or strain arising from physical activity engagement (Robertson \& Noble, 1997).

Self-efficacy - Personal confidence in one's ability to overcome barriers to engage in physically active behaviors, measured using an eight-item questionnaire (Dishman et al., in press).

Step Count - Number of steps accumulated over a period of time, measured in this study by the Walk4Life pedometer.

\section{Significance of the Study}

This study has the potential to impact a number of groups and individuals. On an individual student level, this study may enhance feelings of enjoyment and self-efficacy toward physical activity. It may also heighten students' awareness of physical activity opportunities through DDR that are enjoyable, socially popular, and personally challenging. Ultimately, it may impact their health behaviors in the short and long term. Certainly, the adoption of physically active lifestyles is tremendously important not only to the individual, but society in general.

DDR and Tae-Bo are similar in that they require rhythmic responses to visual and auditory cues. Physiological and psychological responses to each of these modalities may inform researchers, teachers, parents and participants of the applicability of available 
exercise choices. Additionally, an evaluation of these activity types in relation to more traditional course offerings would make educational decisions relevant and meaningful to students. Further, both DDR and Tae-Bo are commercially available products. Widespread use in schools and elsewhere are certainly opportunities worthy of exploration, particularly since these activities do not require gymnasiums or a critical number of participants. The efficacy of DDR in enhancing health-related fitness would be an important finding.

DDR provides students with many choices, not often available in traditional physical education classrooms. Level of difficulty, song choice, and mode of play provide multiple opportunities to engage students and maintain interest for extended periods of time. Few traditional sports activities can provide this level of variation without direct teacher intervention. Skill improvement in DDR is readily apparent, as participants receive immediate feedback following each song.

For teachers, this study can heighten the awareness of different opportunities that exist to integrate a variety of health-related fitness activities in their physical education programs. DDR requires minimal organization and supervision, and this may be attractive for some teachers who may be uncomfortable taking their classes to the gymnasium for more structured "typical" types of physical education activities. Because DDR is safe from a physical education perspective, the teacher can be actively engaged with students, and this may enhance the learning environment and the motivational level of all involved. School wide interventions, mass participation, and competitive opportunities exist with this activity. DDR may also be used as one station in a circuit style physical education class. In addition, DDR is an inclusive activity. For example, 
students with learning disabilities and students in wheelchairs can enjoy this activity. DDR could also serve as an adjunct to physical education programs, thus increasing the physical activity level beyond current levels in the school. DDR may also be offered as a recess activity, although the importance of students engaging in outdoor activities should not be ignored.

For parents, this study may provide them with a safe, physically active, relatively inexpensive family activity that can be enjoyed at home by everyone. All too often, children are permitted to engage in sedentary activities like television viewing and handheld video game play. DDR provides an excellent opportunity to replace those sedentary behaviors with a more active alternative. DDR represents the latest in video technology that children find extremely attractive. The potential for DDR to mask the aversive effects of engaging in structured exercise remains to be explored.

Finally, DDR may positively impact the overall school environment. DDR may play a role in enhancing physical activity levels in school aged children, which reduces stress and increases feelings of self-esteem and well-being (USDHHS, 1996). Many schools are embracing comprehensive school health models that involve all aspects of school life. DDR may become an important part of a school model designed to enhance the total education of the child. 


\section{CHAPTER 2}

\section{REVIEW OF SELECTED LITERATURE}

\section{Introduction}

Decreasing levels of physical activity in children continue to sound the alarm that the future health of North Americans is at risk. Effective interventions are necessary to reverse this trend, and the physical education class is a great place to start. For many children, physical education in schools is the only time they engage in active movement during the day, and it is also an opportune time to reach a captive audience. Today's children are media savvy and responsive to electronic communication in any form. This knowledge can equip physical educators with innovative ideas in which to engage their audience. This chapter is a review of selected literature that is intended to provide the reader with an overview of the following: (1) current levels of physical activity in children and tools to measure physical activity, (2) an examination of the psychological factors influencing physical activity engagement, (3) an analysis of physical activity in physical education classes, (4) an introduction to video game technology and how it can be used to increase physical activity in children, and (5) a summary.

\section{Physical Activity Patterns of Children}

Physical activity is defined as "any bodily movement produced by skeletal muscle that results in energy expenditure" (Casperson, Powell, \& Christenson, 1985, p. 126). Elementary school aged children are more active than their middle and high school counterparts (Guerra et al., 2003; Trost et al., 2002). Boys tend to be more active than girls (Armstrong, Welsman, \& Kirby, 2000; Guerra et al., 2003; Trost et al., 2002). The research regarding the proportion of youth meeting physical activity guidelines is 
equivocal. Some studies report that children meet or exceed the recommended levels of physical activity (Guerra et al., 2003; Sallis \& Patrick, 1994; Sleap \& Tolfrey, 2001). Other studies suggest that the majority of youth are inactive (Armstrong et al., 2000; Trost et al., 2002). In 2003, the Centers for Disease Control (CDC) reported that $61.5 \%$ of children aged 9-13 years did not participate in any physical activity during non-school hours (CDC, 2003). Because insufficient physical activity is a risk factor for overweight, obesity, and related chronic conditions, one of the key objectives of Healthy People 2010 (USDHHS, 2000) is to increase the proportion of active youth. The CDC recommends that children and adolescents participate in at least 60 minutes of moderately intense physical activity on at least five, and preferably all days of the week (USDHHS, 2000). The National Association for Sport and Physical Education (NASPE, 2004) goes further in detailing physical activity recommendations. The following are NASPE guidelines for children's physical activity levels (NASPE, 2004, p. 3-4):

- Children should accumulate at least 60 minutes, and up to several hours, of ageappropriate physical activity on all or most days of the week. This accumulation should include moderate and vigorous physical activity with the majority of the time being spent in activity that is intermittent in nature.

- Children should participate in several bouts of physical activity lasting 15 minutes or more each day.

- Children should participate each day in a variety of age appropriate physical activities designed to achieve optimal health, wellness, fitness, and performance benefits. 
- Extended periods (periods of two hours or more) of inactivity are discouraged for children, especially during daytime hours.

The measurement of physical activity continues to challenge researchers and educators alike. This is particularly true in children, whose physical activity is usually characterized by short, sporadic bursts of energy at a variety of intensities (Bailey et al., 1995). As a result, the accumulation of physical activity tends to receive most attention in the literature as opposed to intensity level (Corbin, Pangrazi, \& Welk, 1994). A number of tools have been used extensively in current research, including criterion standards (e.g. direct observation), objective measures (e.g. heart rate monitors, pedometers, and accelerometers), and subjective techniques (e.g. self-report and interviews) (Sirard \& Pate, 2001; Tudor-Locke \& Bassett, 2004). Each of these methods has inherent limitations and strengths. It is not within the scope of this paper to discuss each of these methods, but simply to determine convergent validity of the tools to be used in this study. A summary of three of these methods (heart rate monitoring, pedometry, and rate of perceived exertion) is presented in the sections that follow.

Heart Rate as a Measure of Physical Activity Intensity

The measure of heart rate continues to be one of the most frequently used methods to estimate exercise intensity. The heart rate monitor, a more recent innovation over the past 25 years, has revolutionized endurance training for athletes. Additionally, the heart rate monitor is finding its way into physical education classes as a means to motivate students, monitor exercise intensity, and establish levels of fitness (Burton, 1996; Kelly, 2000; Martin, Grisson, Ward, \& Lenders, 2003; Schmitt, Peterson, \& Cruz, 2003). As indicated by Gilbert (2005), using maximal heart rate calculations and target 
heart rate zones are problematic in children (ages 5-12) because unlike the adult population, maximal heart rates are age independent. Therefore, the prescription of exercise intensity based on a specific heart rate range is contraindicated in children (Rowland, 1996). The utility of heart rate in this population, however, lies in its ability to compare physical activity levels among different types of activities, and from one exercise period to the next (Kelly, 2000).

Heart rate is measured in beats per minute (bpm) and can be obtained in a variety of ways. The two most common methods used in physical education classes are selfpalpation counting and heart rate telemetry using commercially available heart rate monitors. Self-palpation involves locating the pulse (the pulsations of blood moving through the arteries as the heart muscle contracts) either at the neck (carotid pulse), or the wrist (radial pulse). This method is practical and simple to use, but some measurement error can occur. Erdmann, Dolgener, and Hensley (1998) reported that self-counting techniques consistently underestimated heart rates recorded through heart rate telemetry. These researchers studied middle school-aged boys (mean age 12.7) and used a 15 second pulse count. This finding was earlier reported by Best and Steinhardt (1991) in younger children (third- through fifth-grade), although accuracy tended to improve with age and a shorter measurement interval (e.g. five-second count).

More accurate heart rate monitors have a chest strap containing wireless electrodes that transmit signals to a recording device worn on the wrist (Terbizan, Dolezal, \& Albano, 2002). Other heart rate monitors clip to the earlobe or detect the pulse in the finger (Terbizan et al., 2002). These three types of monitors have a variety of settings that include the longitudinal recording of heart rate over time, programmed 
exercise workouts based on heart rate zones (with auditory cues for the wearer), mean heart rates for a given exercise period, and downloadable data to a computer for storage. The quality of heart rate monitors continues to improve, making them practical tools for the physical education teacher. One drawback for the chest strap monitor, however, is that it requires the participant to undress to place the chest strap next to the skin, and this may present some organizational issues in a physical education setting. The strap must be placed correctly to ensure good contact points for the electrodes, and students must be trained in proper placement. Even so, an active environment like the gymnasium may cause the straps to slip, thus affecting measurement. The Cateye Model PL-6000 (Cateye, Inc.) is a newer model that utilizes an earlobe sensor connected to the recording unit attached to one's belt. Less invasive and more comfortable than the chest strap models, the Cateye monitor provides a convenient alternative heart rate recording. A photograph of the Cateye PL-6000 is presented in Figure 1.

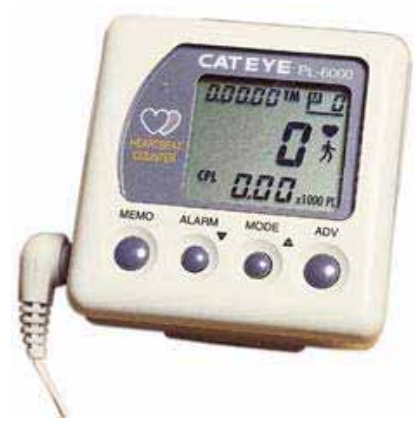

Figure 1. Photograph of the Cateye PL-6000 ear lobe heart rate monitor.

One study (Terbizan et al., 2002) compared the Cateye model and several chest strap models to the "gold standard" in heart rate measurement, namely the electrocardiogram (Achten \& Jeukendrup, 2003). The Cateye was highly correlated with 
electrocardiogram measurements through a range of heart rates. Results are presented in Table 1, and the most popular model for chest strap heart rate monitors (Polar Vantage $\mathrm{XL}$ ) is included for comparison. These data were gathered from a male adult population, and correlations of greater than or equal to 0.90 were considered sufficient to establish validity of the instruments. This standard has been used previously by Leger and Thivierge (1988).

Table 1

Correlations (r) of Two Heart Rate Monitors Compared to an Electrocardiogram Recording

\begin{tabular}{lcccc} 
Monitor & Rest & $85.7 \mathrm{~m} / \mathrm{min}$ & $107.3 \mathrm{~m} / \mathrm{min}$ & $160.8 \mathrm{~m} / \mathrm{min}$ \\
\hline $\begin{array}{l}\text { Cateye PL- } \\
6000\end{array}$ & $r=0.93$ & $r=0.94$ & $r=0.94$ & $r=0.83$ \\
$\begin{array}{l}\text { Polar Vantage } \\
\text { XL }\end{array}$ & $r=0.95$ & $r=0.91$ & $r=0.94$ & $r=0.81$
\end{tabular}

Note. Adapted from "Validity of Seven Commercially Available Heart Rate Monitors," by D.J. Terbizan, A. Dolezal, and C.Albano, 2002, Measurement and Evaluation in Physical Education and Exercise Science, 6, p.246.

Kelly (2000) measured heart rates in 9-10 year old children and collected heart rates continuously over periods of $12-24$ hours. Mean resting heart rates were $72 \mathrm{bpm}$ (range 61-82). This author categorized activity intensity based on heart rate levels relative to resting heart rate. For example, a participant with a resting heart rate of $70 \mathrm{bpm}$, a level 4 heart rate (moderate-vigorous physical activity level) would be in the range of 123-139 bpm. This range was calculated using the estimate of $75-99 \%$ above resting heart rate as 
established by DuRant et al. (1993). Results demonstrated that $90 \%$ of participants achieved moderate-vigorous activity levels for 30 minutes or more on most days of the week. This met the Surgeon General's (USDHHS, 1996) recommendation, but not NASPE's (2004) recommendation of 60 minutes or more, nor the recommendation of Healthy People 2010 (USDHHS, 2000). Burton (1996) also used predetermined heart rate ranges to identify exercise intensity in fifth-grade participants during physical education class. This author reported a mean heart rate of $143 \mathrm{bpm}$, and concluded that heart rates were not sustained sufficiently long enough to increase cardiovascular fitness. Schmitt et al. (2003) reported that sixth-grade boys and girls did not maintain heart rates within target heart rate zones during play in an Ultimate frisbee unit. Johns, Ha, and Macfarlane (2001) categorized heart rates between 139-159 bpm as mild physical activity, and heart rates greater than $159 \mathrm{bpm}$ as moderate-to-vigorous physical activity. Macfarlane and Kwong (2003) reported that children aged 9-11 spent only $16.7 \%$ of their class time within the recommended target heart rate zone of $60-90 \%$ of maximum heart rate. More recently, however, Gilbert (2005) argued that cardiovascular (or aerobic) fitness should focus on improving exercise habits, and not sustaining heart rates within a prescribed zone. NASPE recommendations support this argument, particularly with the statement referring to accumulated physical activity at moderate-vigorous intensities. Therefore, heart rate monitors should be seen as useful tools to monitor physical activity levels in school aged children, not to prescribe exercise intensity toward enhancing cardiovascular fitness. Additionally, heart rate monitors can contribute to the development of healthy exercise habits in youth as well as a greater understanding of the benefits of physical activity. 


\section{Pedometry as a Measure of Physical Activity Intensity}

The pedometer has been established as a convenient, inexpensive means to count steps, and current literature suggests that the pedometer is a reliable, valid tool to gather physical activity data (Kilanowski, Consalvi, \& Epstein, 1999; Rowe, Mahar, Raedecke, \& Lore, 2004; Schneider, Crouter, Lukajic, \& Bassett, 2003; Tudor-Locke, Williams, Reis, \& Pluto, 2004). Electronic pedometers count steps by sensing and recording vertical motions of the hip (Cuddihy, Pangrazi, \& Tomson, 2005). Easily clipped to the waistband, pedometers are small, portable, and unobtrusive, providing the wearer and researcher with a simple means to monitor physical activity. The simplest pedometers record only step counts, whereas more elaborate models record activity time, calories burned, and miles walked. Some pedometers provide auditory feedback (e.g. talking voice), which eliminates the need for the wearer to stop and look at the device during workouts.

Pedometers are gaining popularity for community-based walking programs, personal fitness programs, and health interventions because of their motivational qualities (Tudor-Locke \& Bassett, 2004). The use of pedometers is also a practical method for physical educators to monitor physical activity levels of students (Morgan, Pangrazi, \& Beighle, 2003; Stewart, Dennison, Kohl, \& Doyle, 2004). The growing research into pedometer use demonstrates the potential of these small devices to impact lifestyle physical activity habits in all sectors of the population.

Pedometer studies involving children have only recently been found in the literature. Several studies have analyzed total step counts in children (Cardon \& De Bourdeaudhuij, 2004; Pangrazi, Beighle, Vehige, \& Vack, 2003; Rowlands, Eston, \& 
Ingledew, 1999; Vincent, Pangrazi, Raustorp, Tomson, \& Cuddihy, 2003). These authors reported that boys accumulate more steps per day than do girls. As an example, Vincent et al. (2003) reported that American children aged 6-12 years had mean step counts of 12,554-13,872 for boys and 10,661-11,383 for girls. Pangrazi et al. (2003) demonstrated that a school-based intervention to increase physical activity through 15-minute daily bouts of moderate-vigorous physical activity has a limited impact on daily step counts. This finding, however, demonstrated more effective results when the intervention (PLAY) and physical education class occurred on the same day. There was a more significant impact on step counts of girls than boys in this study. One limitation of this and the other studies is that total step counts do not give information regarding movement intensity. Stewart et al. (2004) reported a mean step count of 1022 for fifth-grade students taking part in a 10 minute bout of moderate-vigorous physical activity during the school day. This would equate to 102 steps per minute, which is much higher than that reported by Scruggs, Beveridge, Watson and Clocksin (2005). These authors examined third- and fourth-grade students engaged in their regular physical education classes, and reported a mean of 64.11 steps per minute. Using an observational instrument to determine total time engaged in MVPA, these authors reported a "cutscore" of 58-61 steps per minute for identifying the achievement of 33\% MVPA during a 30-minute physical education class. Kilanowski et al. (1999) reported a steps per minute value of 41 for 10-year olds engaged in a 57 minute active recreational period.

Pedometers must remain in a vertical position on the waistband to order to accurately register steps (Cuddihy et al., 2005). As a result, pedometers are not indicated for activities such as swimming, cycling, or rollerblading, but they are ideal for weight- 
bearing activities such as walking, jogging or running (Morgan et al., 2003). Pedometers should not be confused with accelerometers, which are more expensive devices designed to measure exercise intensity in both vertical and horizontal planes. One advantage of accelerometers over pedometers is their ability to detect the intensity of movement (Tudor-Locke \& Bassett, 2004). Pedometers simply count steps without discriminating intensity, but more recently, some pedometer models can record time concurrently with step counts, thus providing a more accurate estimate of physical activity intensity (Beets, Patton, \& Edwards, 2005).

Convergent validity (the degree to which pedometer counts associate with other physical activity measures) of pedometers has been established in a meta-analysis done by Tudor-Locke and her colleagues in 2002. These researchers reported that pedometers correlated strongly with accelerometers $(r=0.86)$ and observational methods $(r=0.82)$. The correlation between pedometers and that of self-report instruments varied depending on the particular instrument used $(r=0.02-0.94$, mean $r=0.33)$. In one study specific to young participants (7-12 year-old children), Kilanowski et al. (1999) reported significant correlations between pedometer measures and accelerometer measures $(r=0.99)$, and pedometer measures and observational measures $(r=0.96)$. Tudor-Locke et al. (2002) concluded that pedometers can be used with confidence in research studies involving ambulatory activities, particularly if used in combination with other methods.

A second meta-analysis conducted by Tudor-Locke, Williams, Reis, and Pluto (2004) examined the construct validity of the pedometer. A summary of their findings is presented in Table 2. Age was inversely related to step counts which indicated younger 
participants took more steps per day than their older counterparts. Not one of these studies, unfortunately, included child participants.

Body mass index (the ratio of body weight to height) was also negatively correlated with pedometer step counts. Two studies involving children reported conflicting results, which indicated a possible effect of growth and development in children (Vincent \& Pangrazi, 2002). The fitness indicator 6-minute test revealed a positive relationship, lending further support to the concurrent validity of the pedometer.

Table 2

Correlation Between Pedometer Step Count and Physical Activity Parameters

Physical Activity Parameter Correlation with step count

$\begin{array}{lc}\text { Age } & r=-0.21 \\ \text { BMI (Body Mass Index) } & r=-0.27 \\ \text { Percentage Overweight } & r=-0.22 \\ \text { 6-minute walk test } & r=0.66\end{array}$

Note. Adapted from “Utility of Pedometers for Assessing Physical Activity: Construct Validity,” by C.

Tudor-Locke, J.E. Williams, J.P. Reis, and D. Pluto, 2004, Sports Medicine, 34, 285-288.

Schneider et al. (2003) compared a number of commercially available pedometers. Eight of the ten pedometers tested did not significantly differ from actual step counts. One of the pedometers tested was the Walk4Life LS2525 (Walk4Life Inc., Plainfield, Ill.). This pedometer showed an intramodel reliability of 0.90 (Cronbach's alpha), which indicated little variation in the quality of each pedometer manufactured by this company (Schneider et al., 2003). Beets et al. (2005) tested another Walk4Life 
model, this time the LS 2505. This two-function pedometer (with the same step counting mechanism as the LS 2525) recorded step counts as well as total activity time. An internal stop watch began when the wearer moved, stopped when the wearer stopped, and resumed once again when the wearer moved. As a result, cumulative active time was recorded. This pedometer recorded an overall (e.g. three trials of a one-lap distance around a 400 meter track) intraclass correlation coefficient of 0.99 when compared to actual steps, and 0.997 when compared to actual elapsed time (Beets et al., 2005). This study was conducted with children aged 5-11 years using treadmill walking and selfpaced walking at various speeds. These authors concluded that the added feature of elapsed activity time in addition to step counts gives the researcher much more useful information regarding physical activity levels in children. One limitation cited by these authors was that this measurement tool measured all intensity levels, and did not differentiate low-level intensities from higher-level intensities (Beets et al., 2005). Because of the added feature on this pedometer, and the recently cited reliability of total active time, this pedometer was be chosen for the current study. A picture of this pedometer is presented in Figure 2.

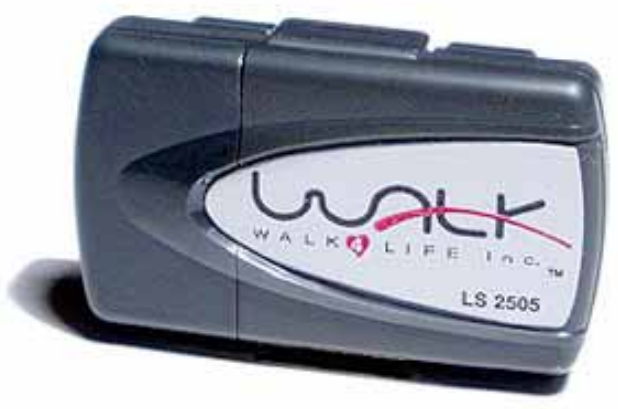

Figure 2. Photograph of the Walk4Life LS2505 pedometer (Walk4Life Inc.). 


\section{Rate of Perceived Exertion as a Measure of Physical Activity Intensity}

The rate of perceived exertion (RPE) has not received much attention other than as a descriptive dependent variable in most studies (Noble \& Noble, 1998). As a result, there is lacking a theory upon which to describe perceived exertion as a physical or psychological construct, or both. The underlying rationale for this construct is based upon Borg's original thesis that perceptual and physiological cues arising from exercise are interdependent (Robertson, 2004). In other words, decisions about the resulting performance are based upon both physiological processes (e.g. pulmonary ventilation, heart rate, and pain) and psychological processes (e.g. pain tolerance, self-efficacy, and mood). The manner in which these processes mediate RPE vary from one individual to the next, yet no consistent pattern has emerged to account for interindividual differences (Robertson, 2004). There is ample evidence to suggest that perceived exertion is a valid and reliable measure of exercise intensity (Noble \& Noble, 1998; Robertson et al., 2005; Yelling, Lamb, \& Swaine, 2002). Since the early work of Borg in the 1960's (who first developed a 15-point graded category scale), a number of researchers have used this template as a means to test a variety of different scales, including some for children (Faigenbaum, Milliken, Cloutier, \& Westcott, 2004). Two such scales seem to have received the most attention in the literature; the Children's Effort Rating Table (CERT) and the Children's Omnibus (OMNI) scale of perceived exertion (Robertson et al., 2000; Williams, Eston, \& Furlong, 1994). The CERT scale was designed as a 10-point scale with 1 being "very, very easy", and 10 being "so hard I am going to stop" (Cowden and Plowman, 1999, p. 34). The conceptual model suggested that heart rate increased linearly with the CERT rating (Williams et al., 1994). Accordingly, heart rate would be 
expressed by the equation: $\mathrm{HR}=100+(10)(\mathrm{CERT}$ number). These researchers found that CERT was not a valid or reliable measure in field settings (e.g. physical education classes) because there was a tendency to underestimate intensity (Cowden and Plowman, 1999). Groslambert, Hintzy, Hoffman, Dugue, and Rouillon (2001) modified the CERT scale by adding pictorial figures. In their study that included children who could not read (mean age 5.5), results indicated significant relationships between heart rate and perceived exertion, but reliability of this scale was low during intermediate stages of running exercise for children in a laboratory setting. Yelling et al. (2002) established the pictorial CERT as a valid measure of exercise intensity in stepping exercise in children aged 12-15 years in a school-based environment. Significant correlations were found between heart rate and CERT scores for all girls, but only for the older boys (ages 14-15). One difficulty with this study, however, was that the researchers used a discontinuous protocol (e.g. a two-minute recovery period between five-minute bouts of stepping exercise), and this may have reduced the perceptions of effort.

The OMNI pictorial 10-point scale has been used in a variety of exercise modalities (e.g. cycling, stepping, running, and walking) and with children 6-13 years of age (Robertson et al., 2005). Utter et al. (2002) established a linear relationship between the OMNI scale and maximal oxygen uptake in walking and running activity in children 6-13 years of age. These researchers also reported significant correlations between the OMNI scale measure and heart rate, as well as other physiological variables. Using mode specific pictures, children selected their perceived exercise intensity during a graded treadmill test. Robertson et al. (2000) established significant correlations between similar measures, but this time for cycling exercise in a mixed gender and mixed race sample of 
8-12 year old children. Robertson et al. (2005) later established construct and criterion validity of the OMNI scale using stepping exercise. One drawback with these studies is that they took place in a laboratory environment, not in a natural setting like a physical education class. The measurement itself is easily implemented with larger groups of students, but the potential social or psychological effects of exercising in the presence of others may impact an individual's perceptions of intensity. The Children's OMNI scale was used in the current study.

Psychological Factors Influencing Physical Activity Behavior Identifying the determinants of physical activity is an important prerequisite for planning and implementing relevant, engaging physical activities for youth (Trost et al., 2001). The complex relationship among environmental, social, biological and psychological factors presents multiple challenges for researchers. This study focused on two key psychological factors; self-efficacy and enjoyment. These physical activity determinants have been cited in the literature as being major positive influences on ones' intention to be physically active (Bungum, Dowda, Weston, Trost, \& Pate, 2000). Sallis, Prochaska, Taylor, Hill and Geraci (1999) found that enjoyment was one of the strongest predictors of physical activity in children in grades 4-12. Trost et al. (1997) reported a strong positive relationship between self-efficacy in overcoming barriers and actual physical activity behavior one year later in fifth grade children. Welk (1999) proposed a theoretical model that blended a number of commonly used theories in the literature to provide a broad perspective on the factors influencing physical activity behavior in children. This model is presented in Figure 3. 


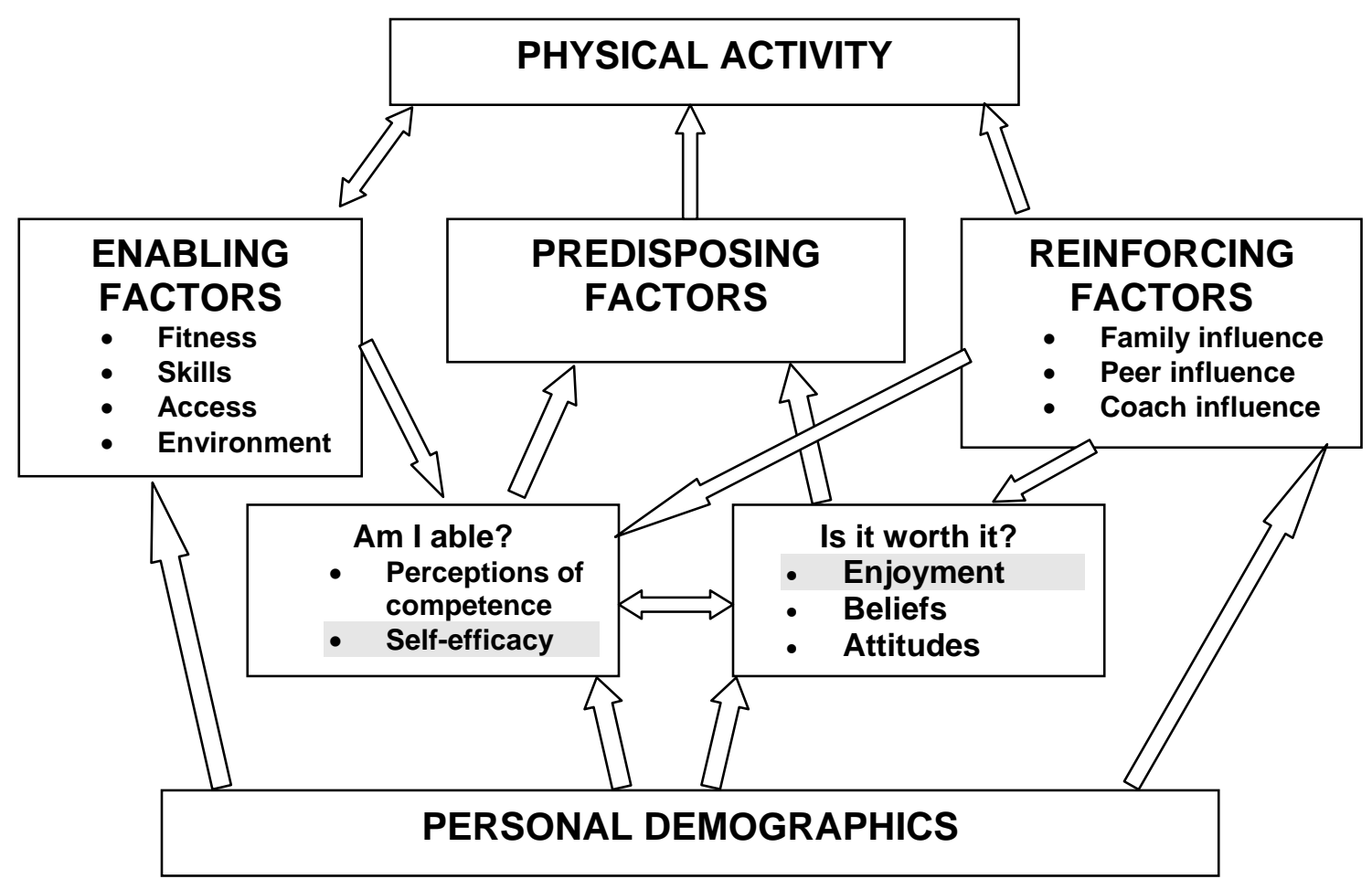

Figure 3. A conceptual diagram of the youth physical activity promotion model. Note.

From "The youth physical activity promotion model: A conceptual bridge between theory and practice”, by G.J. Welk, 1999, Quest, 5 , p. 12. Copyright 1999 by the National Association for Physical Education in Higher Education. Reprinted with permission.

For the purposes of this study, the predisposing factors of self-efficacy and enjoyment were analyzed. Predisposing factors are antecedents to behavioral change, and they "include variables that collectively increase the likelihood that a person will be physically active on a regular basis" (Welk, 1999, p. 11). Welk (1999) proposed that an individual asks two key questions when deciding upon physical activity behavior, and these are, “Am I able?", and "Is it worth it?” (p. 13). The first question identified self- 
efficacy as a determining factor. Children will usually pursue activities in which they believe they can be successful. The second question addressed cognitive and affective factors such as attitudes, beliefs, and enjoyment. Welk (1999) suggested that there were strong links between these two categories of predisposing factors. This study examined his assumption.

\section{Enjoyment of Physical Activity}

Enjoyment of physical activity is a significant correlate with the intention to engage in moderate-vigorous physical activity for both males and females (Bungum et al., 2000). Additionally, enjoyment provides immediate reinforcement for being physically active (Motl et al. 2001), and, according to Dishman et al. (2005), enjoyment of physical activity may also have an indirect influence on self-efficacy. It makes sense, then, that enjoyment of physical activity may influence the adoption of exercise as a lifestyle habit (Kimiecik \& Harris, 1996).

In a longitudinal study of children's enjoyment of physical education, Prochaska et al. (2003) found a consistent decline in enjoyment of physical education from fourth grade to sixth grade (though not significant). This was particularly true for girls in this sample. In this study, enjoyment was assessed using a one-question instrument with pictorial and numerical codes (e.g. $1=$ frowning face and $6=$ smiling face). Though the researchers were cognizant of the potential reliability and validity problems with a onequestion instrument, they reported significant correlations between enjoyment scores and mile-run times. Macfarlane and Kwong (2003) also used a single item five-point Likert scale (e.g. "How did you like the physical activities today?"; $5=$ excellent, $1=$ poor, p. 181). These researchers noted lower levels of enjoyment in Hong Kong children than 
American children of the same age, but this was attributed to the highly structured classes and lack of program variety.

Kendzierski and DeCarlo (1991) designed an 18-item instrument called the Physical Activity Enjoyment Scale (PACES). These authors report that this instrument had high internal consistency and test-retest reliability, although this instrument was designed using undergraduate college students. In a subsequent study by Crocker et al. (1995), a Cronbach's alpha of 0.90 for internal consistency was reported. This study involved sport camp participants aged 12-16 years, and they engaged in sport-related activities (e.g. track, basketball, soccer, and tennis). It can be argued that a sport camp setting is quite different from that of a physical education class, so the generalizability of results comes into question. Motl et al. (2001) used PACES with a group of adolescent girls in eighth grade. These authors modified the instrument to better reflect this age group, and the rating scale was reduced from a 7-point bipolar scale to a 5-point Likert scale. Through exploratory and factor analysis, researchers concluded that PACES consisted of a single factor (e.g. enjoyment), and it provided better support for the validity of this instrument in this age group (Motl et al., 2001). The validity of this instrument was further confirmed by Dishman et al. (2005) in eighth-grade girls using an intervention-based study over the course of one year. Baseline measures were gathered during eighth grade, and once again following a one-year intervention concluding in ninth grade. The intervention was designed to promote physical activity through a choice based, gender sensitive program. This study reported that enjoyment had a moderate effect on self-efficacy but a weak effect on physical activity. The authors suggested that enjoyment predicted self-efficacy, and therefore, indirectly increased physical activity 
among adolescent girls (Dishman et al., 2005). Subsequent to this research, Dishman et al. (in press) reported further refinement of the PACES instrument to include only negatively worded items. This was done to eliminate the methodological effect of positively worded items reported by Motl et al. (2001). Dishman et al. (in press) included sixth- and eighth-grade girls in this test-retest design, and results further validated the modified PACES as an appropriate instrument to use with middle school children. Self-Efficacy for Physical Activity

Self-efficacy has been defined as a judgment of one's ability to perform a task within a specific domain (Bandura, 1977, 1997). A major tenet of his social cognitive theory, Bandura (1989) has espoused the concept of self-efficacy as "a major function of thought.” (p. 1176). Accordingly, self-efficacy influences behavior. In the field of physical education, for example, higher self-efficacy beliefs will lead to better performance than low self-efficacy beliefs. Not to be confused with outcome expectancy, which is the belief that certain behaviors will lead to certain outcomes, self efficacy is the degree to which one believes in his or her ability to successfully perform the behavior in question (Feltz \& Chase, 1998). Consequently, performance success raises self efficacy beliefs, and failure tends to lower beliefs (Chase, 1998). Performance and self-efficacy, therefore, affect one another. Performance begets self-efficacy, and similarly, selfefficacy begets performance. Thought patterns can aid or hinder performance, and the degree of self-efficacy for a specific context will determine the direction of those thought patterns. Bandura (1989) suggested that we shape our experience of our environment based on our self-efficacious thoughts: the environment, the situation, or other people do not shape us. 
The role of self-efficacy in teaching and learning continues to interest practitioners and researchers alike (Woolfolk Hoy, 2000). The importance of considering the ability level of the individual in light of the individual's belief that he or she will succeed at a particular task remains central to teaching and learning (Nietfeld \& Enders, 2003). By providing students with appropriate learning experiences in which students will have some success, self-efficacy will be enhanced, and thus, the likelihood that students will want to engage in those experiences more frequently (McAuley \& Mihalko, 1998).

Sallis, Prochaska, and Taylor (2000) reported equivocal findings in their review of children's physical activity determinants involving self-efficacy as one of the measures. In part, this was due to the type of self-efficacy being measured. According to Bandura (1997) two main types of self-efficacy behaviors are most predictive of physical activity; task-related self-efficacy and self-regulatory efficacy (McAuley \& Mihalko, 1998). The task-related construct refers to the belief in one's ability to be physically active for a given period of time, intensity and duration, and the self-regulatory construct refers to overcoming barriers in order to be active (Ryan \& Dzewaltowski, 2002).

In a study conducted by Simons-Morton et al. (1997) self-efficacy was significantly related to time spent engaged in moderate-to-vigorous and vigorous physical activity. The results of this study were supported by Bungum et al. (2000) using ninthgrade males and females as participants. Both of these studies examined task-oriented self-efficacy. Physical activity behavior was predicted successfully in a study by Trost et al. (1997) in preadolescent children. Trost et al. (2001) reported that self-efficacy levels differed significantly between obese and non-obese fifth grade children. In this study, 
obese children also demonstrated significantly lower levels of daily physical activity as compared to non-obese children. Obese children were significantly less confident in their ability to overcome barriers to physical activity than their non-obese counterparts, thus partially explaining the differing levels of physical activity in these two groups. Thompson, Romanow, and Horne (1998) examined intentions to be active in children aged 7-11 years. These researchers observed and coded physical activity behaviors on three separate occasions during physical education classes over the course of a 10 -week period using video tape analysis. In addition, children were given self-efficacy questionnaires at the beginning of each session. Self-efficacy was found to be the most significant predictor of intention to be active when self-efficacy scores measured immediately before the activity were compared to subsequent activity in class.

Dishman et al. (in press) reported that "barriers" self-efficacy and physical activity are directly related in a sample of sixth-grade girls. In this study, an eight-item questionnaire was used on two separate occasions, two weeks apart to determine stability of instrumentation. The five-point rating scale identified the degree to which the individual agreed with the statement (e.g. "I can be physically active during my free time on most days no matter how busy my day is", p. 5). Unlike the Thompson et al. (1998) study, which examined changes in self-efficacy over time, this study simply administered the questionnaires during physical education class.

\section{Physical Activity in Physical Education}

Ever since Anderson and Barrette (1978) published their findings on how students spend their time in physical education class, much research has focused on strategies to enhance physical activity levels. These authors reported that students spend at least $60 \%$ 
of their time in physical education class waiting or receiving information. Twenty-two years later, McKenzie, Marshall, Sallis, and Conway (2000) reported similar findings: middle school students spent approximately $14.6 \%$ of physical education classes engaged in moderate-vigorous physical activity. An additional $33.8 \%$ of the time was spent walking. Given that most physical education classes are no more than 30 minutes in length, and many schools average less than three periods per week, physical education classes are not providing the minimum recommended levels of physical activity for children (McKenzie et al., 2000; Trudeau \& Shephard, 2005). Similarly, Levin, McKenzie, Hussey, Kelder and Lytle (2001) reported that students were engaged in moderate-vigorous physical activity in $34.9 \%$ of fourth grade physical education lessons and $42.4 \%$ of fifth grade lessons. Since physical education class may be the only opportunity for children to be physically active, these data should be cause for concern (Pate et al., 1995; Sallis \& McKenzie, 1991). Increasingly, teachers are recognizing the need for health-related physical activity programs in schools due to rising obesity levels and changing leisure time habits of children (Portman, 2003).

Many high quality physical education programs exist. One such example was noted by Sallis et al. (1997) in which a health-related physical education program called Sports, Play, and Active Recreation for Kids (SPARK) demonstrated significant increases in physical activity levels during class. In physical education classes taught by Physical Education specialists, children in fourth-grade engaged in moderate-to-vigorous physical activity $40.2 \%$ of the time. This was compared to $17.8 \%$ in the control classes, and $32.7 \%$ in classes taught by trained classroom teachers. The SPARK program focused on healthfitness activities such as aerobic dance, jump rope, and walking/jogging, and skill-fitness 
activities such as basketball and soccer. The goals of this program were to promote high levels of physical activity by providing enjoyable activities for all children, as well as provide an opportunity for children to enhance their movement abilities. These authors (Sallis et al., 1997) concluded that a health-related physical education curriculum, supported by qualified teachers, had tremendous potential to contribute to health-related benefits in children. The SPARK curriculum has seen widespread implementation across the United States, and McKenzie et al. (2000) more recently reported that this curriculum was successful in increasing levels of moderate-to-vigorous physical activity (MVPA). However, gender differences were more noticeable in middle school grades (e.g. boys more active than girls). In order to address this concern, an examination of curricular content may be appropriate to determine what types of activities are most enjoyable to both genders of middle school students.

Fitness activities elicit the greatest energy expenditures in elementary school children (McKenzie et al., 1995), and it is these types of activities that contribute most to health related benefits. According to NASPE (2005), health-related components of fitness include aerobic fitness, muscular strength and endurance, flexibility, and body composition. It is generally accepted that aerobic fitness is the most important, since the health benefits of this type of activity reduce cardiovascular disease risk factors (Sallis \& McKenzie, 1991). In fact, physical activity recommendations from the CDC and NASPE are focused exclusively on aerobic fitness. The most frequent physical education activities reported by teachers include fitness activities such as calisthenics, jumping rope, and jogging in addition to sport-oriented activities like soccer and basketball (Ross, Pate, Corbin, Deply, \& Gold, 1987). NASPE (2005) recommended continuous training, 
circuit training, station learning, and interval training to enhance aerobic fitness. Although recent literature is lacking with respect to the use of commercially available exercise videos in physical education classes, anecdotal evidence suggests that teachers do consider their use on occasion. Very popular in the 1980's, exercise videos contain several segments in which each of the components of health-related fitness was addressed: warm up and flexibility, aerobic fitness, muscular endurance, and cool down with more flexibility. The video selected for this study was entitled Tae-Bo, an introductory workout for beginners. The complete workout was 36 minutes in length, and included each of the components listed above. Wood (1986) presented some recommendations for the use of such videos with children. She suggested that the adultoriented aerobic dance workout is inappropriate for children in the fourth grade and younger, but for older children exercise videos could be one way to enhance aerobic fitness. Wood did caution that aerobic exercise research in children was not substantive. NASPE (2005) recommended intermittent bouts of activity 10-20 minutes for the age group used in this study, so the Tae-Bo video, if used in part, was appropriate. The use of an exercise video in this study determined its applicability in fifth- and sixth-grade physical education classes. Similar to DDR, Tae-Bo invited the participant to perform rhythmic movements while following the cues on the television monitor. Unlike DDR, however, the participant did not receive any feedback on his or her successful performance other than positive reinforcement from the teacher. Tae-Bo also limited the choices available to the participant in terms of song selection and bodily movements. The participant chose the intensity and accuracy with which he or she wished to respond to 
the leader on the screen. Both Tae-Bo and DDR used video and audio technology to engage participants, so the comparison between the two was most interesting.

Volleyball is a popular curricular activity in most physical education programs. Generally considered less active than other fitness oriented activities because of the emphasis on skill development, volleyball can still contribute to health-related fitness. NASPE (2005) stressed the importance of age-appropriate activities that contributed to physically active lifestyles. The popularity of volleyball and the intermittent nature of game play meet this recommendation. A well-balanced physical education program would include a wide variety of activities, and volleyball is an appropriate choice. In this study, volleyball was the activity chosen and scheduled by the teachers at that particular time of year.

\section{Video Games}

\section{Video Game Use}

Traditional video games (e.g., those using a hand held controller or joystick) play an important role in the lives of many children, youth, and adults. By the early 1980's, video games joined television as the most popular leisure time activity for children (Provenzo, 1991). Even infants and children are not immune to the pervasiveness of electronic media exposure. On any given day it is estimated that $68 \%$ of children under the age of two will sit in front of a television set, computer or video game for an average of just over two hours (Rideout et al., 2003).

Funk (1993) reported that $63 \%$ of girls and $88 \%$ of boys in seventh and eighth grade played at least one to two hours per day of video games at home. The favorite video game categories were those including some degree of violence (e.g., fantasy 
violence, sports, human violence). Educational games were popular in only $2 \%$ of cases. Physically active video games were not part of Funk's study because this type of game had not yet entered the video game market. Funk also suggested that video game play habits may reflect future behaviors in some children or adolescents.

Haninger and Thompson (2004) reported that over $90 \%$ of teen-rated video games released by 2001 had violent content. Anderson (2004) established a significant link between exposure to violent video games and aggressive behavior. In this meta-analysis of current research, Anderson found this link to be causal, and suggested that previous studies may have underestimated the true magnitude of effects on behavior, cognition, and affect. Emes (1997), however, pointed to video game violence as abstracted aggression whereas the violence on television often depicted reality. He also suggested that video game play provided an opportunity for players to release pent-up energies through physical action and concentration. In his review, Emes pointed to the contradictory evidence of short-term aggressive behavior resulting from video game play, and emphasized that none of the studies examined revealed harmful emotional or psychological effects of video game play. Nevertheless, many parents and educators are concerned about the content of video games and the potential impact they may have on the physical and psychological development of children.

Another issue surrounding video game use is the sedentary nature of this pasttime. The "couch potato" hypothesis proposes that time spent playing video games and watching television is thought to displace physical activity and is therefore related to obesity in children (Gortmaker et al., 1990; Robinson, 2001). Few studies have examined video game use specifically, but in one study, McMurray et al. (2000) found no 
significant relationship between obesity and video game use in 10-17 year olds.

Kautiainen et al. (2005) more recently reported no significant association between overweight and digital game play. Digital game play included video, computer and console games. In this study, 14-18 year old participants were surveyed according to their digital game play, television viewing and computer use (emailing, surfing, etc.). Television viewing was positively associated with overweight in both boys and girls but computer use was only associated with overweight in girls. The authors suggest that playing digital games may differ from television viewing in their effects on physical activity or eating habits.

Body Mass Index (BMI) is often used to assess body fatness in children. This measure is gender and age specific because of growth changes occurring in the childhood years (Pietrobelli et al., 1998). A ratio of body weight to height, the CDC (2001) has developed a BMI-for-age gender specific charts in which a percentile ranking can be obtained for each child. The corresponding weight status category based on percentile ranking is used widely as a screening tool by health care practitioners to encourage lifestyle changes (CDC, 2001). The CDC (2001) weight status categories are as follows; underweight (less than $5^{\text {th }}$ percentile), healthy weight $\left(5^{\text {th }}\right.$ percentile up to the $85^{\text {th }}$ percentile), at risk of overweight ( $85^{\text {th }}$ percentile to less than $95^{\text {th }}$ percentile), and overweight (equal to or greater than the $95^{\text {th }}$ percentile).

In a nationally representative study, Vandewater et al. (2004) found a significant relationship between video game use and obesity in children aged 1-8. Using 24 hour time-use diaries, these researchers more accurately obtained physical activity data than had been done in the past and used BMI as a measure of obesity (Pietrobelli et al., 1998). 
While no causal link is made, Vandewater et al. (2004) speculated that overweight or obese girls in particular may turn to video games because of social isolation. The direction of causality is certainly an interesting topic for further research. If video game use continues to be implicated as one of many factors contributing to the obesity epidemic, the introduction of physically active video games may circumvent one likely causal agent.

\section{Physically Active Video Games}

Physically active video games are breaking new ground in the gaming industry. Methods of play now combine physical body movements with mental processes resulting in much higher levels of physical activity. Many of these games are sports oriented, where players must kick a soccer ball, box with a virtual opponent, or skateboard through an obstacle course. Created by Japanese video game giant Konami, Inc. in 1998, Dance Dance Revolution ${ }^{\circledR}$ has become extremely popular in Japan and is quickly gaining momentum here in the United States. In January 2005, worldwide sales exceeded 7.5 million units (“Konami Ships Dance Dance Revolution Ultramix3 to Retail Stores Nationwide", 2005). Both arcade and home versions are commercially available at retail outlets across the United States. The arcade version became available in the United States in 2000 and the home version a year later. Compatible with both Microsoft's Xbox ${ }^{\circledR}$ and Sony's PlayStation ${ }^{\circledR} 2$, the home version of DDR will track the number of calories burned, the number of steps taken, and total workout time. A variety of settings within the game provide multiple ways in which to play the game. These include workout mode, game mode, and battle mode, among others. As a result of the growing popularity of the game, many DDR competitive tournaments are appearing all over the United States. 
Xbox games include an online version in which players can compete against others around the world.

Unlike other interactive games, DDR relies on rhythm and beat for successful game play. The desire to interact with music is one reason why this game is so popular. This game appeals to all age levels, and many adults are recognizing the value of DDR as one way to exercise at home. Testimonial evidence suggests that many people have used DDR as a means to lose weight (Schnitzler, 2001).

In a recent study using DDR, Tan et al. (2002) measured the energy cost and cardiorespiratory responses to arcade game play in a sample of teenagers. Subjects were given two weeks of unlimited access to the game prior to data collection. Difficulty level was self-selected, and measures were taken during six songs (approximately 10 minutes) played in arcade mode in which grade scores were given at the conclusion of each song. The researchers reported that participants reached mean heart rates similar to mediumintensity exercise (137 beats per minute). This study concluded that DDR was sufficiently vigorous enough to meet the ACSM minimum recommended guideline for developing aerobic fitness. In a study at The Pennsylvania State University, Yang and Graham (2005) reported that DDR could promote moderate to vigorous levels of physical activity. In this ongoing study, the authors noted heart rates in the range of 100-160 beats per minute. Participants 12-18 years of age were members of a local YMCA. They were given 45 minutes to play DDR as much as they wanted. Heart rate monitors and accelerometers were used to gather physical activity data. The participants also reported high levels of enjoyment despite the elevated heart rates. In a self-report study, Smith (2005) tried a variety of physically active video games, DDR being on of them, and 
claimed that game play alone improved his health significantly. He noted that these types of games were far more engaging than the traditional exercise videos, and therefore were more likely to have a lasting impact on fitness. As an information technology professor, Smith also discussed how these games could easily be modified to better meet physical activity guidelines, which include; warm up and cool down activities, target heart rate zones (sustained game play), and monitoring calories burned. It should be noted that the Xbox version of DDR will track calories burned as game play continues.

Anecdotal evidence suggests that DDR can play a role in reducing the obesity epidemic in the United States. Students at the University of Hawaii have played DDR in a campus arcade and reported significant weight loss (Schnitzler, 2001). At a recent International Consumer Electronics Show in Las Vegas ("Play to Get Fit", 2005), company executives suggested that most people do not even recognize they are exercising. This so called "exergaming" may unwittingly engage the country's youth in behaviors that could result in health-related benefits. While not a panacea, this type of video gaming may be one way to address the obesity problem.

DDR has been used in the treatment of physical and mental disabilities. Testimonial evidence from a mother of a child with hypotonia (decreased muscle tone) revealed that DDR significantly enhanced her son's rhythmic ability such that he no longer lagged behind other children his age (Brobst, 2002).

An exploratory study of sixth-grade students with dyslexia and ADHD (attention deficit hyperactive disorder) revealed that a four-week DDR school intervention significantly enhanced reading and attentional skills because the visual and auditory demands of DDR strengthened the neural connections involved in these cognitive skills 
(McGraw et al., 2002). Students attended twice weekly 25 minute DDR sessions while at school. These researchers suggested that the use of DDR in educational settings warranted further research, and that DDR may play a role in the pursuit of valid educational goals, particularly in addressing the disruptive nature of ADHD behaviors. Another study conducted on boys with ADHD, aged 6-16 years, found that when engaged in a motivating activity like a video game, ADHD symptoms were much less apparent (Houghton et al., 2004). Although this study used a different video game (Crash Bandicoot) that did not involve large body movements, a high degree of motor control and concentration was necessary for successful game play. Also noted was the opportunity for these students to be more interactive with their peers in social settings like video game environments. "Computer games offer teachers, clinicians, and parents increased opportunities to assist children with ADHD to maximize their learning potential" (Houghton et al., 2004, p. 32).

Few studies exist on this relatively new video game format, but Ridley and Olds (2001) examined the energy cost of interactive video. They found moderate to high energy expenditures in both male and female participants (mean age 12.5) at a video game center. The energy expenditure was related to walking or jogging ranging in speeds from 1.9-4.7 miles per hour. Though DDR was not included as one of the games, several games involved large upper and lower body movements. The authors were quick to caution that their results were based on at least five minutes of sustained activity, which may not necessarily have represented typical video game center behavior. Players typically play sporadically, and spend substantial amounts of time socializing with 
friends. Nonetheless, their results indicated the potential for these type of video games to increase physical activity levels in children.

\section{Summary}

The health related benefits of regular physical activity are well documented (Sallis et al., 2000). The concern that many children are not meeting recommended physical activity guidelines has motivated many researchers to examine the factors influencing levels of activity engagement as a means to design appropriate intervention strategies (Simons-Morton et al., 1997). The measurement of physical activity therefore is of paramount importance in determining the effectiveness of such interventions. Pedometers and heart rate monitors are two reliable and readily available objective tools to measure physical activity levels in children (Sirard \& Pate, 2001). While pedometers do not provide information regarding exercise intensity, they do measure accumulated step counts and active time. Heart rate monitors provide more specific evidence of exercise intensity over a period of time. Additionally, these devices are relatively unobtrusive, easy to use, and inexpensive assessment tools appropriate for use with children.

Perceived exertion scales provide researchers with an additional valid measure of exercise intensity (Robertson et al., 2005). The Children's OMNI scale is easily understood by young children, and it can be implemented with large groups. It has demonstrated significant positive correlations with heart rate (Utter et al., 2002), and has been used successfully in a variety of exercise settings (Robertson et al., 2000; Robertson et al., 2005; Utter et al., 2002). 
The psychological factors influencing physical activity behavior are multifaceted and complex. Two important determinants of physical activity engagement are selfefficacy and enjoyment (Dishman et al., 2005). It is relevant to examine both constructs, and the potential relationship between the two given a variety of physical education activities. Enhancing both self-efficacy and enjoyment of physical activities in physical education are salient issues for teachers hoping to foster in their students the development of physically active lifestyles.

The potential for interactive video games to impact physical activity levels in children has not yet been explored. There is no question that youth today are highly motivated by the video game format, so an opportunity exists to capitalize on their interest to engage them in a physically active pursuit. It is quite possible that children may accrue health related benefits using DDR, and not even realize their perceptions of exertion.

As teachers continue to seek new and interesting activities to meet their objectives for physical education, DDR may provide them with one more tool to increase fitness levels, and perhaps more importantly, help students gain a better appreciation for the importance of a physically active lifestyle. DDR is one such activity that can be played throughout life, individually or in groups, and at a self-prescribed intensity. Because it can be individualized, it can meet the needs of all students. This game merits investigation, not only because it appeals to students (of all ages), but because it also reflects society's preoccupation with technology. 


\section{CHAPTER 3}

\section{METHOD}

The overall purpose of this study was to examine the effects of the interactive video game DDR on the following measures in elementary school children: heart rate, rate of perceived exertion, step counts, activity time, self-efficacy, and enjoyment. In comparing DDR to Tae-Bo and regular physical education (volleyball) classes across all dependent variables, the degree to which DDR met MVPA guidelines was determined. This chapter is organized into several sections, which include: participants; research design; instrumentation; procedures; hypotheses; and data analysis.

\section{Participants}

Two elementary school classes were involved in this study; one sixth-grade class and one fifth-grade class. Classes were recruited from one school in the Near North District School Board in North Bay, Ontario, Canada. This is a small public school board in Northern Ontario. A letter of permission to approach Near North District Schools is attached in Appendix A. Initially there were 22 students in fifth-grade and 25 students in sixth-grade for a total of 47 participants. Due to absenteeism, injury, excused absences, and equipment problems, the results of 33 participants were used for the purposes of this study. Selected demographics (age, gender, height, weight, body mass index) of the participants were gathered using the form attached in Appendix B, and are presented in Table 3. Participants did not receive any monetary incentives for their participation. Participants and their parents were presented with full details of the study prior to their agreement to participate. A letter accompanied the consent form sent home with each 
student two weeks prior to the beginning of the study. Parents were invited to an information evening one week prior to the beginning of data collection.

\section{Table 3}

Mean Age, Height, Weight and Body Mass Index ( \pm Standard Deviation) of Fifth- and Sixth-Grade Participants.

\section{Grade 5}

Females
Males $(\mathrm{n}=13)$
Grade 6

Females Males

Total

Variable

$$
(\mathrm{n}=8)
$$$$
(n=5)
$$

$(\mathrm{n}=7)$

$(\mathrm{N}=33)$

$\begin{array}{lccrrr}\text { Age (years) } & 10.75 \pm .71 & 10.46 \pm .52 & 12.00 \pm .00 & 11.86 \pm .90 & 11.06 \pm .899 \\ \text { Height }(\mathrm{cm}) & 153.86 \pm 7.95 & 144.12 \pm 8.11 & 154.50 \pm 5.57 & 151.24 \pm 10.04 & 149.56 \pm 9.08 \\ \text { Weight }(\mathrm{kg}) & 46.09 \pm 13.45 & 42.45 \pm 13.32 & 52.58 \pm 7.66 & 45.59 \pm 12.98 & 45.53 \pm 12.52 \\ \text { Body Mass } & 19.25 \pm 3.89 & 20.12 \pm 4.81 & 22.14 \pm 3.65 & 19.83 \pm 5.67 & 20.15 \pm 4.53 \\ \text { Index } & & & & & \end{array}$

Institutional Review Board for the Protection of Human Rights (West Virginia University) and Ethics Committee (Nipissing University) approval were obtained prior to collecting data on human participants. West Virginia IRB approval for exempt status was obtained, and a copy of this approval can be found in Appendix C. A signed copy of Ethics Committee (Nipissing University) approval is attached in Appendix D. Participant assent and parental/guardian informed consent was required for Nipissing University, and the necessary forms are attached in Appendices E and F, respectively. Signed consent forms and assent forms were received from all parents and participants. In the interests of 
confidentiality, participant identification was protected in this study. Each participant was assigned a code number, and that number was used for the duration of the study. Participants recorded only code numbers on all questionnaires and scales throughout the study. During data analysis, all identifying information was removed, and data were stored in a locked, secure cabinet in the researcher's office.

\section{Research Design}

The experimental research design was a repeated measures within-subjects format. The independent variable was activity type. The three levels of the independent variable were Dance Dance Revolution (DDR), Tae-Bo, and regular physical education (volleyball). Each participant engaged in each of these activities for 20-30 minutes on each of six occasions for a total of 18 lessons (e.g. 6 x DDR, 6 x Tae-Bo, 6 x volleyball). The ordering of the blocks of the three different activities was assigned based on a table of random numbers. The random ordering counterbalanced the repeated measures, and the order was the same for both classes for ease of implementation. For example, the second block (three days of 30 minutes, 30 minutes, and 30 minutes) was Tae-Bo (Thursday), DDR (Monday), and volleyball (Wednesday). The third block was Tae-Bo (Thursday), volleyball (Monday), and DDR (Wednesday). Thus, in 18 sessions, each participant had six DDR sessions, six volleyball classes, and six Tae-Bo classes. A sample ordering for repeated measure for the intervention is illustrated in Table 4. The independent variables are noted in Table 5. The classroom teacher led each session, so two different teachers (e.g. fifth-grade and sixth-grade classroom teachers) were involved in the lessons. 
Table 4

Random Orders of Activity Type within Blocks

\begin{tabular}{|l|c|c|c|}
\hline Block 1 & Day 1 & Day 2 & Day 3 \\
\cline { 2 - 4 } & Volleyball & Tae-Bo & DDR \\
\hline Block 2 & Day 4 & Day 5 & Day 6 \\
\cline { 2 - 4 } & Tae-Bo & DDR & Volleyball \\
\hline Block 3 & Day 7 & Day 8 & Day 9 \\
\hline Block 4 & Tae-Bo & Volleyball & DDR \\
\hline Block 5 & Day 10 & Day 11 & Day 12 \\
& DDR & Volleyball & Tae-Bo \\
\hline Block 6 & Day 13 & Day 14 & Day 15 \\
& Volleyball & DDR & Tae-Bo \\
\hline & Day 16 & Day 17 & Day 18 \\
\hline & DDR & Tae-Bo & Volleyball \\
\hline
\end{tabular}

Both teachers and participants underwent familiarization sessions for DDR and Tae-Bo. Teachers were given a game system (Xbox, game CD, dance pad) to take home for a period of weeks to become comfortable with DDR. Each teacher was provided with a lesson plan for both types of lessons. The lesson plans are included in Appendix G. Instructions for use of DDR, the pedometers, and the heart rate monitors are also included in Appendix G. The participants were given two familiarization sessions for DDR and Tae-Bo. These two sessions, scheduled during physical education classes, involved a DDR station, a Tae-Bo station, and a volleyball station. Students rotated to each of the 
three stations. Additionally, teachers introduced the pedometers during the first familiarization session, and the heart rate monitors during the second familiarization session. The volleyball lessons were prepared by the classroom teacher, and these lessons were typical to what would have been presented under normal circumstances. Two sample lesson plans are included in Appendix G. The researcher was engaged in ongoing planning sessions with the teachers involved. Prior to data collection, the researcher and the teachers finalized implementation procedures, which will be detailed in the following sections.

The researcher had extensive experience teaching DDR to school-aged children as part of an ongoing research project at West Virginia University. This study involved the same type of pedometer used in the current study. Additionally, the researcher had an opportunity to implement all current study instruments in one secondary school physical education class. It was therefore anticipated that methodological concerns were addressed adequately through prior experience and ongoing discussions with the classroom teachers. The familiarization period contributed to the preparation for data collection in terms of distribution and use of heart rate monitors and pedometers, equipment set up in the gymnasium, and removal of pedometers and heart rate monitors immediately following each exercise session.

The dependent variables for this research design were (1) heart rate, (2) step counts, (3) active time, (4) rate of perceived exertion (RPE), (5) enjoyment, and (6) selfefficacy. A summary of the dependent variables is illustrated in Table 5.

Heart rate is defined as the number of beats per minute. Heart rate was measured using Cateye-PL-6000 heart rate monitors clipped to the earlobe, and heart rates were 
recorded every 30 seconds during the exercise period so that average heart rate was displayed and recorded at the end of each exercise session. The heart rate monitor also displayed elapsed time, so at the conclusion of each physical education class, students were asked to stop the timer. This permitted the recording of total time for each class.

\section{Table 5}

Independent and Dependent Variables

Independent Variable: Activity Type

Dance Dance Revolution

Volleyball

Tae-Bo

Dependent Variables:

Heart Rate

Step Count

Percent Active Time

Rate of Perceived Exertion

Enjoyment

Self-efficacy

Step counts were measured using the Walk4Life 2505 Pedometer (Walk4Life, Inc), placed horizontally on the waistband of each participant. A safety strap added additional security in case the pedometer became unclipped. These devices also recorded active time. Immediately following each activity session, participants removed their 
pedometers and carefully placed them on the stage in the gym before they returned to their classroom. This stopped the recording of steps and active time. Both step count and active time were then manually recorded for each participant on a data sheet prepared for that purpose.

Rate of Perceived Exertion (RPE) is defined as one's personal judgment of effort based on changing body sensations during exercise. RPE was measured using the Children's OMNI Perceived Exertion Scale, as this scale has been well validated in the literature (Robertson et al., 2005). Permission to use this scale was obtained by the author, and is included in Appendix H. This 10-point numerical and pictorial scale permitted students to simply circle the appropriate number corresponding to their perceived exertion immediately after each exercise session. A sample of this scale is attached in Appendix I. Participants were given a personal copy of the OMNI Scale to complete at the conclusion of each physical education class.

Self-efficacy describes the personal belief in one's ability to accomplish a specific task (Bandura, 1997). The self-efficacy scale developed by Dishman et al. (2005) was used for this study. This eight-item scale contained a five-point Likert rating scale to determine the degree to which the individual agreed with each item. Total scores were recorded for each participant and a score of 40 was the highest score possible. A higher score indicated a greater level of self-efficacy. The self-efficacy instrument was implemented once at the beginning of the study, and once again at the conclusion of the study. This instrument was given to participants at the same time of day in both situations on a day that did not include physical education class. Permission to use this scale was 
obtained by the researcher and is included in Appendix J. A sample of the self-efficacy scale is attached in Appendix K.

Enjoyment can be defined as a positive affective state that reflects feelings of pleasure (Motl et al., 2001). Enjoyment as a dependent variable was measured using the PACES (Physical Activity Enjoyment Scale) designed for this age group (Dishman et al., 2005). This instrument was based on a 28 item, five-point Likert rating scale asking the participant to rate "enjoyment" on a scale from 1 (Dislike a lot) to 5 (Enjoy a lot). Based on more recent findings, Dishman et al. (in press) has noted that removal of all positively worded items eases the implementation of the scale. Additionally, Motl et al. (2001) noted that positively worded items affected the validity of the instrument in their study of adolescent girls. As a result, the instrument used in this study included a seven-item scale with negatively worded items in which participants were asked to rate the degree to which they agreed with each statement on a five-point Likert rating scale. The highest score possible was 35. A sample of the enjoyment scale is included in Appendix L. Participants were asked to complete this scale immediately after each activity period (concurrent with the OMNI scale). Permission to use this instrument has been obtained, and is presented in Appendix $\mathrm{J}$.

\section{Instrumentation}

A demographic questionnaire was utilized to gather general information about each participant. Information about age, gender, experience with DDR, and level of physical activity was gathered with this instrument. This provided the researcher with some qualitative data on each participant that may have impacted the variables being studied. Body height and weight were measured by the researcher to obtain Body Mass 
Index of all participants. The same commercially available weigh scale was used both pre- and post-intervention. Height and weight measures were obtained in the same location, separate from the rest of the other participants. A sample of the demographic questionnaire, as previously noted, is attached in Appendix B.

\section{Heart Rate}

Heart rate was measured using the Cateye-PL-6000 heart rate monitor. This device was worn on the waistband with an earlobe sensor attached with a wire connection. The monitor was also attached to a belt worn around the waist to ensure the instrument remained attached at the waist. This monitor has been shown to accurately measure heart rates when compared to the "gold standard" electrocardiogram (ECG) (Terbizan et al., 2002). Correlation coefficients $(r)$ between the recorded heart rate using the Cateye monitor and the corresponding ECG Heart rate exceeded 0.90. As a result, the authors considered this monitor a valid instrument. This conclusion is supported by Leger and Thivierge (1988), who conducted earlier research in heart rate monitor validity. All participants wore their own heart rate monitor during each session, and they wore the same monitor each time. All monitors were numbered. These devices enabled the researcher to gather heart rate data for the whole exercise session (mean heart rate for the 20-30 minute period), as well as total elapsed time. These data were recorded immediately following each exercise session.

\section{Step Count and Active Time}

Each participant was given a pedometer to be placed on the waistband prior to each exercise session. Pedometers were re-set to zero for each session so cumulative step counts and total active times were recorded following each session. Participants used the 
same instrument for each trial. Active time accumulated only when the participant was stepping, and time accumulation stopped when stepping stopped. As a result, total active time reflected total stepping time. The Walk4Life 2505 Pedometer (Walk4Life, Inc.) was used in this study, as it remains a reliable, valid instrument to determine both step counts and total active time (Beets et al., 2005). This pedometer recorded an overall intraclass correlation coefficient of 0.99 when compared to actual steps, and 0.997 when compared to actual elapsed time (Beets et al., 2005). These researchers established the intraclass coefficients during treadmill walking at four different speeds for children aged 5-11. Schneider et al. (2003) used a similar model developed by the same company (with the identical internal mechanism) and reported an intramodel (e.g. from one pedometer to another) Cronbach's alpha reliability of 0.90 during walking on an outdoor track in adult subjects. This indicated that the Walk4Life pedometers are appropriate instruments for research into physical activities involving walking in children (Beets et al., 2005).

\section{Rate of Perceived Exertion}

Perceived exertion is defined as one's subjective rating of exercise intensity based on body sensations (Noble \& Noble, 1998). This investigation used the Children's OMNI Perceived Exertion Scale as developed by Robertson et al. (2000). This scale uses pictorial and numerical descriptions of exertion on a graded slope. The pictures can be modified to reflect the type of exercise being performed. For example, Robertson et al. (2005) used stepping exercise; Robertson et al. (2000) used cycling exercise; and Utter et al. (2002) used walking and running exercise. This study utilized depictions of an individual walking and running (Appendix I). Concurrent validity coefficients for this instrument were in the range of $r=0.81$ to $r=0.94$ (Robertson et al., 2005) when 
compared to heart rate and oxygen uptake. While not as high, significant positive correlations were found between the walking/running instrument and maximal oxygen uptake and heart rate (Utter et al., 2002). Construct validity for the stepping instrument ranged from $r=0.93$ to 0.95 (Robertson et al., 2005) when compared to the cycling OMNI scale. These authors suggest that the OMNI Perceived Exertion Scale can be used over a wide range of nonweight-bearing and weight-bearing physical activities for children 8-12 years of age. Utter et al. (2002) found the walking/running OMNI scale to be strongly correlated with percent oxygen uptake in children aged 6-13, with $r$ values ranging from .41-.60. Participants in the present study identified their rate of perceived exertion immediately following each physical education class.

\section{Enjoyment}

Enjoyment of physical activity relates to exercise adherence (Kendzierski \& DeCarlo, 1991), and can be defined as the positive feelings resulting from participation. The Physical Activity Enjoyment Scale (PACES) is an 18-item inventory using a sevenpoint Likert scale for each item. The PACES instrument has been shown to have high internal consistency. Using Cronbach's coefficient alpha, Kendzierski and DeCarlo (1991) reported a value of .96 for both exercise cycling and jogging on a trampoline. This internal consistency coefficient was later confirmed by Crocker et al. (1995) with an alpha of .90. In that study, 12-16 year old male and female participants engaged in track and field, basketball, soccer, and tennis. Motl et al. (2001) have more recently provided evidence of construct validity through confirmatory factor analysis of 4 different models depicting hypothesized relationships among factors influencing enjoyment, physical activity, and sport involvement. These researchers examined a modified instrument that 
was used in a sample of eighth-grade girls. In this instrument, the rating scale was changed to a five-point Likert scale that was easier to understand, and they rewrote some of the items to make them more relevant to this age group. More recently, Dishman et al. (in press), successfully used this instrument for sixth-grade girls. Although the validity of the instrument was not tested, the authors noted a positive correlation between selfefficacy and enjoyment $(r=.40)$. In this study, enjoyment was measured using PACES immediately following each physical education class. Both the perceived exertion scale and the enjoyment scale were placed on the same piece of paper, and once completed, each participant submitted his or her own copy to the teacher. Self-Efficacy

Self-efficacy is a major determinant of physical activity engagement (Sallis et al., 2000). Dishman et al. (in press) developed an eight-item self-efficacy questionnaire for use with eighth- and ninth-grade girls making judgments about overcoming barriers to being physically active. These authors have also successfully used it with sixth-grade girls. The reliability coefficient (Cronbach's alpha) for this instrument was found to be 0.79. The eight-item measure of self-efficacy was found, through factor analysis, to be a valid construct in the measure of self-efficacy. This was confirmed over a two-week physical activity intervention in which self-efficacy demonstrated a direct relationship with physical activity. This brief questionnaire (Appendix K), which focuses on overcoming barriers to physical activity, was distributed at the beginning of the study, and once again at the conclusion of the study. Participants completed the questionnaires in their classroom at a time separate from physical education class, and submitted completed questionnaires to the classroom teacher. 


\section{Procedure}

Written approval from the Director of Education of the Near North Board of Education was obtained, and a copy of the letter is attached in Appendix A. Verbal approval from the principal of the school involved was secured prior to this study. Institutional Review Board (West Virginia University) and Ethics Committee (Nipissing University) approval was obtained prior to data collection to ensure the protection of Human Subjects (Appendices C \& D). Once all necessary approvals were received, participants, parents and teachers were encouraged to participate in the study. The researcher met with the two teachers involved on several occasions to inform them fully of the scope of the study. Their responsibilities were explained and both teachers were very supportive and eager to participate. Operational issues regarding management of equipment, transfer of equipment from one grade to the next, completion of questionnaires, and any other potential issues affecting implementation were discussed, and any issues resolved prior to data collection. Parents and participants were invited to an information meeting to explain the purposes of the study and to familiarize them with the procedure. Additionally, this meeting was used as an opportunity for parents and participants to complete the demographic questionnaire. Incentives were offered to return the consent and assent forms in a timely manner. Any parent not wishing his or her child to participate was not penalized. Only one parent refused consent, but the parent did permit his daughter to participate in all activities as long as no data were collected. This student was not assigned a pedometer or a heart rate monitor, nor did she complete any of the questionnaires. 
The teachers involved with each class were asked to perform all teaching duties during the study. Each teacher was familiarized with the study protocol, and was trained to implement both the DDR and the Tae-Bo lessons. Written lesson plans and discussions with the researcher prepared the teachers to feel comfortable with lesson content. Teachers were also responsible for ensuring participants correctly placed the pedometers and heart rate monitors. Although the researcher was present for each session to assist in equipment distribution and set up, troubleshooting during lesson implementation, and equipment collection, she did not take a leadership role in lesson implementation. Volleyball lessons were presented by the classroom teacher as she normally would. Although neither teacher was a physical education specialist, both teachers were responsible for physical education throughout the year. As a result, both teachers were experienced and comfortable in meeting Ontario curriculum expectations in physical education. Lesson guidelines were provided for each DDR and Tae-Bo session, and teachers were asked to follow the same protocol each time. Samples of these can be found in Appendix G. Each lesson plan contained 30 minutes of content that included at least 20 minutes of active time. The remaining time was used for preparation, organization, questionnaire completion (enjoyment scale and OMNI perceived exertion scale) and lesson closure.

As previously indicated, participants were introduced to each activity type before the series of lessons began. A certain level of competence was required to perform DDR and Tae-Bo continuously so it was anticipated that an orientation period of two sessions would provide the necessary training for all students. Participants became comfortable with both DDR and Tae-Bo on these occasions. On one additional occasion, DDR was 
presented as an activity on a day in which few students were at the school (snow day), so some students had more practice with DDR. Students had had volleyball classes during previous years and were therefore already familiar with some of the activities and skills to be presented in the volleyball lessons. During the familiarization period, students were provided with an opportunity to work on volleyball skills. Participants were introduced to the pedometers and heart rate monitors prior to the data collection, and they used the pedometers and heart rate monitors separately and together during the familiarization lessons. These sessions proved to reduce participant reactivity to the instruments and eased the implementation of data collection once the study began.

Information regarding height, weight, current activity level, and experience with DDR was collected prior to the beginning of the study (during the familiarization period) using the data sheet presented in Appendix B. This same information was gathered once again at the conclusion of the study using the same equipment and same location. Additionally, participants were asked to record their best grade score and level of difficulty for DDR (e.g. grade score of "A" at difficulty level "Light").

\section{Heart Rate Monitors and Pedometers}

All heart rate monitors and pedometers were numbered and assigned to specific participants. As an example, heart rate monitor \#1 and pedometer \#1 were assigned to participants 501 (fifth-grade participant) and 601 (sixth-grade participant). Each participant used the same equipment each time, so a maximum of two participants used each set of equipment. All heart rate monitors worked reliably for the duration of the study, and only three pedometers had to be replaced due to battery failure. It was not anticipated that this adversely affected the results since the intramodel reliability of the 
pedometers was reported to be very high (Schneider et al., 2003). The heart rate monitors were not sensitive enough to detect all heart beats of some participants. As a result, any heart rate below 80 beats per minute was discarded. Since the average resting heart rate was 79 beats per minute, acceptance of values at or above 80 beats per minute was deemed appropriate.

Heart rate monitors and pedometers were distributed in the classroom prior to movement to the gymnasium. Teachers ensured that monitors and pedometers were placed properly. Pedometers were placed horizontally on the waistband, and the safety clip attached to the clothing. Heart rate monitor belts were clipped around the waist, the unit was also attached to the waistband of clothing, and the earlobe sensor was passed under clothing and clipped to the earlobe. Some participants chose to have the sensor wire on the outside of their clothing. At that point, participants turned on the heart rate monitors to display current heart rate (memory function not yet activated). The pedometers were not sealed, but had to be closed to work properly. Participants could not therefore check their progress until the end of the lesson. The heart rate monitors were not enclosed in a pouch, so participants could check their heart rates during the lesson. This resulted in more consistent data since participants were asked on occasion to check to be sure that the instruments were working properly, and the researcher was able to circulate throughout the class to make quick visual checks of the instruments. Once students arrived at the door of the gymnasium or were seated inside the gymnasium awaiting further instructions, the teacher asked students to re-set their pedometers to zero, and activate their heart rate monitors (by pressing one button). The gym class began immediately following this point. The researcher circulated throughout the gymnasium to 
check for proper placement and functioning of the equipment. At the conclusion of each class, participants were asked to stop the heart rate monitor by pressing one button, and subsequent to that, participants removed both the pedometer and heart rate monitor and carefully placed both on the stage. Participants filed out of the gymnasium to their classroom and completed the OMNI perceived exertion scale and the enjoyment scale. The fifth-grade class had physical education class from 12:45-1:15, and the sixth-grade class from 1:45-2:15. During the interim, the researcher recorded step counts, active time, heart rates, and elapsed time from all instruments, and re-set instruments in preparation for the sixth grade class. She then delivered the instruments directly to the sixth-grade classroom for subsequent distribution. One assistant aided in the recording of some of the pedometer data, and the researcher recorded the rest of the pedometer data and all of the heart rate monitor data. All data from the pedometers and the heart rate monitors were subsequently transferred onto a Microsoft Excel spreadsheet.

\section{Questionnaires and Scales}

As soon as participants entered the classroom from the gymnasium, they were asked to complete the OMNI perceived exertion scale and the PACES questionnaire (both on the same sheet of paper). Teachers clarified any confusion over the wording, and consistently emphasized the importance of honesty and thoroughness in completing the questionnaire. Participants identified their papers using code numbers only, and all questionnaires were collected by the teacher and returned to the researcher before she left the school each day. The researcher checked to ensure that she had all questionnaires, and that they were completely filled out. Any questionnaire with missing information was returned to the participant for completion before the researcher left the school that day. 
The self efficacy questionnaires were distributed by the classroom teacher during regular class time (same time of day for both pre- and post-intervention). Again, the teacher explained and clarified any difficult wording, and encouraged participants to answer as honestly as possible. These questionnaires were collected by the teacher and passed along to the researcher after they were completed. All data from questionnaires were recorded and checked by the researcher, and entered on a Microsoft Excel spreadsheet.

\section{DDR Sessions}

All DDR sessions took place in the gymnasium. Dance pads were stored on a wheeled cart on the stage (in the gymnasium), and the televisions and Xboxes were stored on wheeled carts in a secure room. The television carts were moved to the gymnasium by the researcher prior to each DDR session. Television monitors were arranged in a circle in the middle of the gymnasium with the screens facing outward. This formation worked well to manage the noise level and it maximized space for the dance pads. Four dance pads were plugged into each unit. Once participants entered the gymnasium, they were instructed to activate their pedometers and heart rate monitors and choose one dance pad. Participants were free to choose their own groups of four, and there were sufficient pads for all participants. Participants were encouraged to continue play for the entire gym class. Once a song was selected, participants followed the cues on the screen for the duration of that song, and thus were active for approximately 90 seconds. A brief stoppage in game play allowed participants to receive their grade score (e.g. A-E) and make a new song selection. This time period was approximately 15-30 seconds. Song selection was rotated among all four participants at each television 
monitor, and choice was up to the individual. All participants were given an opportunity to choose at least two songs. The DDR game contains over 75 songs, so song selection was quite variable. Many participants would choose favorites, while other participants sought to try new songs each time. Song selection was not controlled by the teacher or the researcher. Difficulty level was also self-selected (e.g. Beginner, Light, Standard, or Heavy). Volume was controlled by the researcher and the teachers. On two occasions, a dance pad malfunctioned, but the researcher was able to diagnose and correct the problem with little disruption in data collection. Each X-Box was set to complete each song regardless of the success of the participants, so once a song was selected, 90 seconds of active time ensued. Participants recorded their highest grade attained for that period once back in the classroom. The teacher's role during these sessions was to encourage students to continue play for the entire time and to answer any questions which participants might have had. With the intermittent nature of DDR, participants completed approximately 1015 songs within the allotted time period.

Once the fifth-grade class had completed their DDR lesson, they filed out of the gymnasium. The equipment was left in the gym awaiting the sixth-grade class. Once the sixth-grade class ended, the researcher, with assistance from several students, dismantled the DDR equipment and stored it once again.

\section{Tae-Bo Sessions}

All Tae-Bo sessions occurred in the gymnasium. This commercially available exercise DVD included boxing-like maneuvers, marching actions, and stretching exercises. The researcher moved one television monitor (with DVD player) to the gymnasium for this purpose. After two sessions, two television monitors linked together 
were used to spread the participants throughout the gym. This set-up was used for the remainder of the study. The teacher was asked to use different parts of the DVD for each session. For example, sessions one and two began at the start of the video (warm up, stretching, cardiovascular fitness), sessions three and four began later on (cardiovascular fitness, muscular endurance), and sessions five and six were similar to the first two sessions. Participants were asked to simply follow the leader on the video, and encouraged by the teacher to participate for the entire lesson. A sample of the general lesson outline is included in Appendix G. Once the Tae-Bo lesson ended, the researcher returned the equipment to the storage room.

\section{Volleyball Sessions}

These sessions all took place in the gymnasium. Nets were set up in advance of the class. The classroom teacher presented her normally scheduled physical education curriculum during this time. She prepared and implemented her own lesson plan for each of these sessions. Two samples are presented in Appendix G. Different types of activities were presented, and these included warm up activities, skill building activities and game play. In five of the six sessions, two volleyball nets were used. In the fourth session, only one net was used. Students were arranged individually, in pairs, small groups, and teams at various points during each lesson. Students were instructed on skill performance, particularly on volleying and bumping, and were encouraged to participate fully for the entire lesson. Most lessons had two distinct phases; skill development and game play. Lessons for both grade levels were similar in this respect. 


\section{Research Hypotheses}

Based on current research literature and the intuitive judgment of the researcher, the following hypotheses were proposed for this intervention:

1. Participants engaging in DDR will demonstrate similar heart rates compared to regular physical education (volleyball) and Tae-Bo classes.

2. Participants engaging in DDR will demonstrate similar step counts and active time compared to regular physical education and aerobic dance classes.

3. Participants engaging in DDR will demonstrate lower rates of perceived exertion for a given heart rate than for Tae-Bo or volleyball.

4. Participants engaging in DDR will demonstrate higher levels of enjoyment compared to volleyball or Tae-Bo.

5. Participants will demonstrate higher levels of self-efficacy at the end of the study than at the beginning.

\section{Data Analyses}

Means and standard deviations of descriptive variables of participants were presented in tabular form, and included height, weight, body mass index, and resting heart rate. Pre- and post-intervention means were reported for comparison. A paired samples $t$ test was performed to determine significant changes in body mass index over the course of the six week intervention. Body mass indices were grouped into one of four categories, according to the CDC guidelines (2001). These categories were; underweight $\left(<5^{\text {th }}\right.$ percentile), healthy weight $\left(\geq 5^{\text {th }}\right.$ and $<85^{\text {th }}$ percentile $)$, at risk of overweight $(\geq$ $85^{\text {th }}$ percentile and $<95^{\text {th }}$ percentile $)$, and overweight $\left(\geq 95^{\text {th }}\right.$ percentile $)$. Data were presented in a frequency table. For the paired samples $t$ test, the first two categories were 
collapsed as well as the last two categories to form two distinct groups according to body mass index; those at or below a healthy weight, and those at risk of overweight or overweight. This was done to maintain statistical power for the sample size.

A frequency table was also used to present data relating to physical activity levels of participants. The categories identified in this table included pre- and post-intervention self-reported physical activity on weekdays and weekends (not including physical education class). Additionally, participant experience with DDR was presented as a frequency table.

A $3 \times 6$ repeated measures within-subjects analysis of variance (ANOVA) was performed on each of the following dependent variables; heart rate, step count, percent active time, rate of perceived exertion and enjoyment. There were three levels of the first independent variable (volleyball, Tae-Bo, and DDR) and six levels (trials) of the second independent variable. Table 6 displays a sample of the repeated measures procedure for one of the dependent variables (heart rate). Multiple comparison post hoc paired sample $t$ tests were performed to identify within-subject effects across trials and activity types.

Table 6

Sample 3 x 6 Repeated Measures Analysis of Variance for Heart Rate (HR)

\begin{tabular}{lllllll}
\hline & \multicolumn{7}{c}{ Trials } \\
\hline Activity Type & 1 & 2 & 3 & 4 & 5 & 6 \\
Volleyball & HR1 & HR2 & HR3 & HR4 & HR5 & HR6 \\
Aerobic Dance (Tae-Bo) & HR1 & HR2 & HR3 & HR4 & HR5 & HR6 \\
DDR & HR1 & HR2 & HR3 & HR4 & HR5 & HR6
\end{tabular}


Overall means for each activity type (trials were collapsed together) were calculated and compared using paired sample $t$ tests. Tables and graphs were prepared to present data as appropriate. Subsequent between-subjects repeated measures analyses of variance were performed for each of the following dependent variables; heart rate, step count, percent active time, rate of perceived exertion, and enjoyment. There were three levels of the independent variable (activity type), and participants were blocked according to gender, grade level and BMI. Each block had two groups, each defining the between-subject independent variables. The two groups for each block were; male and female (gender), fifth-grade and sixth-grade (grade level), and $<85^{\text {th }}$ percentile and $\geq 85^{\text {th }}$ percentile (BMI). This analysis of variance was referred to as a split plot factorial 222.3 ANOVA. Subsequent post hoc independent $t$ tests were conducted on significant ANOVAS. A sample split plot factorial 222.3 repeated measures ANOVA is presented in Table 7. In addition, Pearson product-moment correlation coefficients were conducted on dependent variables to determine significant relationships between variables.

Table 7

Sample Split Plot Factorial 222.3 Repeated Measures ANOVA for Heart Rate (HR)

\begin{tabular}{lllllll}
\hline & \multicolumn{2}{c}{ Gender } & \multicolumn{2}{c}{ Grade Level } & \multicolumn{2}{c}{ BMI Category } \\
\cline { 2 - 6 } Activity Type & Male & Female & $5^{\text {th }}$ grade & $6^{\text {th }}$ grade & $\begin{array}{c}<85^{\text {th }} \\
\text { percentile }\end{array}$ & $\begin{array}{c}\geq 85^{\text {th }} \\
\text { percentile }\end{array}$ \\
\hline Volleyball & HR & HR & HR & HR & HR & HR \\
Tae-Bo & HR & HR & HR & HR & HR & HR \\
DDR & HR & HR & HR & HR & HR & HR
\end{tabular}


Active time was measured by the pedometer and recorded in seconds. Total elapsed time was recorded by the heart rate monitor and was also recorded in seconds. Percent active time was calculated for each participant in each activity type across all six trials in the following manner: active time/elapsed time $\mathrm{x} 100$. Active time, expressed as a percent, was subjected to the same analyses as the other dependent variables.

Step count was also expressed as steps per minute to further determine exercise intensity. This was calculated by expressing step count as a fraction of total elapsed time for each lesson, and reported as a rate per minute. Subsequent paired samples $t$ tests were performed among all three activity types.

A paired samples $t$ test was also conducted for self-efficacy pre- and postintervention. Self-efficacy was measured on a five-point Likert scale for a maximum possible score of 40 points. Results of the $t$ test were presented in tabular form.

Pearson's correlation coefficients were calculated among the dependent variables to determine the degree of linear association between them.

SPSS version 13.0 for Windows was used for all statistical analyses. A significance level of .05 was used in all ANOVAS, paired samples $t$ tests, and independent samples $t$ tests. Microsoft Excel was used to prepare all graphs.

Scores for the dependent variables noted above were obtained as follows:

1. Heart Rate was measured in beats per minute (bpm).

2. Step Counts were the total number of steps accumulated during each trial.

3. Active Time was expressed as a percent of total elapsed time.

4. Rate of Perceived Exertion was based on a score selected on a scale of 0-10. 
5. Enjoyment scores were obtained using a Likert scale of 1-5 on each of seven items for a maximum possible score of 35 .

6. Self-efficacy scores were obtained using a Likert scale of 1-5 on each of eight items for a maximum possible score of 40 . 


\section{CHAPTER 4}

\section{RESULTS}

The research hypotheses for this study were as follows:

1. Participants engaging in DDR will demonstrate similar heart rates compared to regular physical education and aerobic dance classes.

2. Participants engaging in DDR will demonstrate similar step counts and active time compared to regular physical education and aerobic dance classes.

3. Participants engaging in DDR will demonstrate lower rates of perceived exertion for a given heart rate than for aerobic dance or regular physical education.

4. Participants engaging in DDR will demonstrate higher levels of enjoyment compared to regular physical education or aerobic dance.

5. Participants will demonstrate higher levels of self-efficacy at the end of the study than at the beginning.

This chapter is organized in order of the hypotheses. Descriptive data will be presented first, followed by heart rate data, step count and active time data, perceived exertion data, enjoyment data, and self-efficacy data.

\section{Demographic Data}

The data were gathered over a six week period (January $26^{\text {th }}$-March $8^{\text {th }}, 2006$ ). Forty-seven students were enrolled in this study. Due to absenteeism, injury, and heart rate monitor insensitivity, 14 of the original 47 sets of data were deemed unusable. As a result, 33 sets of data are presented from 21 fifth-grade participants and 12 sixth-grade participants. The sample was made up of 13 (eight from fifth-grade, five from sixthgrade) females and 20 (13 from fifth-grade, seven from sixth-grade) males, and mean age 
was 11.06 years. Selected descriptive data were presented in the previous chapter in

Table 3. Pre-and post-intervention descriptive data are presented in Table 8. Body mass indices were calculated using the standard formula provided by the CDC (2001).

Table 8

Pre- and Post-intervention Descriptive Data in Fifth- and Sixth-Grade Participants, $N=33$

\begin{tabular}{ccccc}
\hline Variable & Minimum & Maximum & Mean & $\begin{array}{c}\text { Standard } \\
\text { Deviation }\end{array}$ \\
\hline Height - Pre $(\mathrm{cm})$ & 132.20 & 172.00 & 149.56 & 9.08 \\
Height - Post $(\mathrm{cm})$ & 133.20 & 172.00 & 150.28 & 9.11 \\
Weight - Pre $(\mathrm{kg})$ & 24.70 & 76.10 & 45.53 & 12.52 \\
Weight - Post $(\mathrm{kg})$ & 25.00 & 74.30 & 46.38 & 12.73 \\
BMI - Pre & 14.20 & 28.20 & 20.15 & 4.53 \\
BMI - Post & 14.10 & 29.00 & 20.30 & 4.51 \\
Resting Heart Rate (bpm) & 53.00 & 98.00 & 79.09 & 12.63
\end{tabular}

Table 9 presents body mass index in terms of weight category status guidelines of the National Center for Health Statistics (2005), and frequencies of each of the four categories are separated by gender. Using the above noted guidelines, $39 \%$ of participants in this study were either at risk of overweight or overweight. For the purposes of data analysis, BMI groups were collapsed into two groups: healthy and/or underweight $\left(<85^{\text {th }}\right.$ percentile), and, risk of overweight and overweight ( $\geq 85^{\text {th }}$ percentile). 
Table 9

Body Mass Index (BMI) Frequencies in Fifth- and Sixth-Grade Participants.

$\begin{array}{clcc}\text { Underweight } & \text { Healthy Weight } & \text { At Risk of } & \text { Overweight } \\ \left(<5^{\text {th }}\right. & \left(\geq 5^{\text {th }} \text { and }<85^{\text {th }}\right. & \text { Overweight }\left(\geq 85^{\text {th }}\right. & \left(\geq 95^{\text {th }}\right.\end{array}$

Gender

Percentile)

Percentile)

and $<95^{\text {th }}$ Percentile)

Percentile)

\begin{tabular}{lcccc}
\hline Females $(n=13)$ & 0 & 9 & 2 & 2 \\
Males $(n=20)$ & 2 & 9 & 2 & 7 \\
\hline Total & 2 & 18 & 4 & 9 \\
Percent $(\%)$ & $6 \%$ & $55 \%$ & $12 \%$ & $27 \%$
\end{tabular}

Participants were asked to report their daily levels of physical activity (not including physical education class). These data were collected pre- and post-intervention, and are presented in Table 10. Paired samples $t$ tests were conducted to determine any significant change in the activity level of participants from pre- to post-intervention. The result of this paired $t$ test, $t(32)=-.947, p>.05$, was not significant. The second paired $t$ test. The result of this $t$ test, $t(32)=-1.437, p>.05$, was not significant. As indicated by these results, there were no significant changes in activity level (not including physical education class) during the six week intervention. 
Table 10

Frequency Distribution of Physical Activity on Weekdays and Weekends in Fifth- and Sixth-Grade Participants, $N=33$

Minutes of Physical Activity

$\begin{array}{lcccc}\text { Variable } & \text { None } & 10-30 \text { minutes } & 30-60 \text { minutes } & >60 \text { minutes } \\ & & \text { daily } & \text { daily } & \text { daily } \\ \text { Weekdays - pre } & 1 & 12 & 6 & 14 \\ \text { Weekdays - post } & 1 & 7 & 10 & 15 \\ \text { Weekends - pre } & 2 & 6 & 13 & 12 \\ \text { Weekends - post } & 1 & 6 & 10 & \end{array}$

Over half $(52 \%)$ the participants had never tried DDR prior to the intervention. Table 11 shows the distribution of participant experience with DDR. One participant already had the game at home, and she played DDR on a regular basis. One participant was given the home version of the game during the intervention, and he began to play DDR regularly after three weeks of the intervention. 
Table 11

Frequency Distribution of DDR Experience in Fifth- and Sixth-Grade Participants

Experience with DDR

\begin{tabular}{ccccccc} 
& Never & $1-2$ & $3-10$ & $>10$ & Daily & Total \\
& Times & Times & Times & & $N$ \\
\hline Frequency & 17 & 6 & 4 & 5 & 1 & 33
\end{tabular}

Heart Rate

Mean heart rates were recorded for each participant during each exercise session (trial). The heart rate monitors recorded participant heart rate once every 30 seconds, and the overall mean heart rate for the session was calculated and displayed on the instrument. The overall mean heart rate for each trial was recorded by the researcher. Heart rate data including means and standard deviations are presented in Appendix N. A $3 \times 6$ repeated measures within-subjects analysis of variance was conducted in the following manner; the independent variables were activity type (3 levels), and trials (6 levels), and the dependent variable was heart rate. This revealed only a main effect for activity type, $F(2,64)=4.988, p<.05$. There was no main effect for trials, $F(5,160)=.607, p>.05$, nor was there an interaction between activity type and trials, $F(10,320)=1.526, p>.05$. Because there was no interaction between activity type and trials as a result of the $3 \times 6$ repeated measures ANOVA, multiple comparisons on the activity main effect were computed and are reported later in this section. Figure 4 
illustrates the mean heart rate per trial across all activity types.

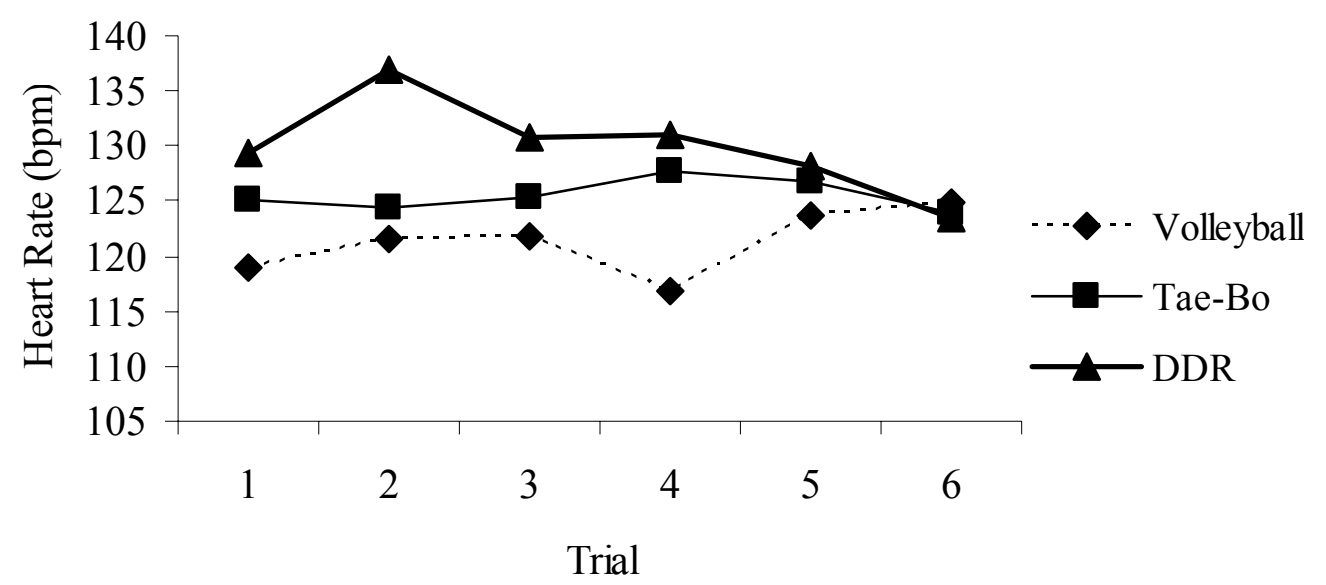

Figure 4. Mean heart rate per trial for all participants. No significant interaction was found between activity type and trial.

When heart rate was collapsed across trials, an overall mean for each activity type was determined. Mean heart rates measured in beats per minute (with standard deviations in parentheses) for volleyball, Tae-Bo and DDR were 121.27 (12.01), 125.59 (13.11), and 128.83 (13.23), respectively. Multiple comparisons for the significant main effect for activity type were conducted to determine differences among means. Significant differences were noted between volleyball and DDR, as displayed in Table 12. Figure 5 graphically displays the means and standard deviations of heart rate for each activity type used for the $t$ test results in Table 12 . 
Table 12

Multiple Comparisons for the Significant Main Effect of Activity Type on Heart Rate

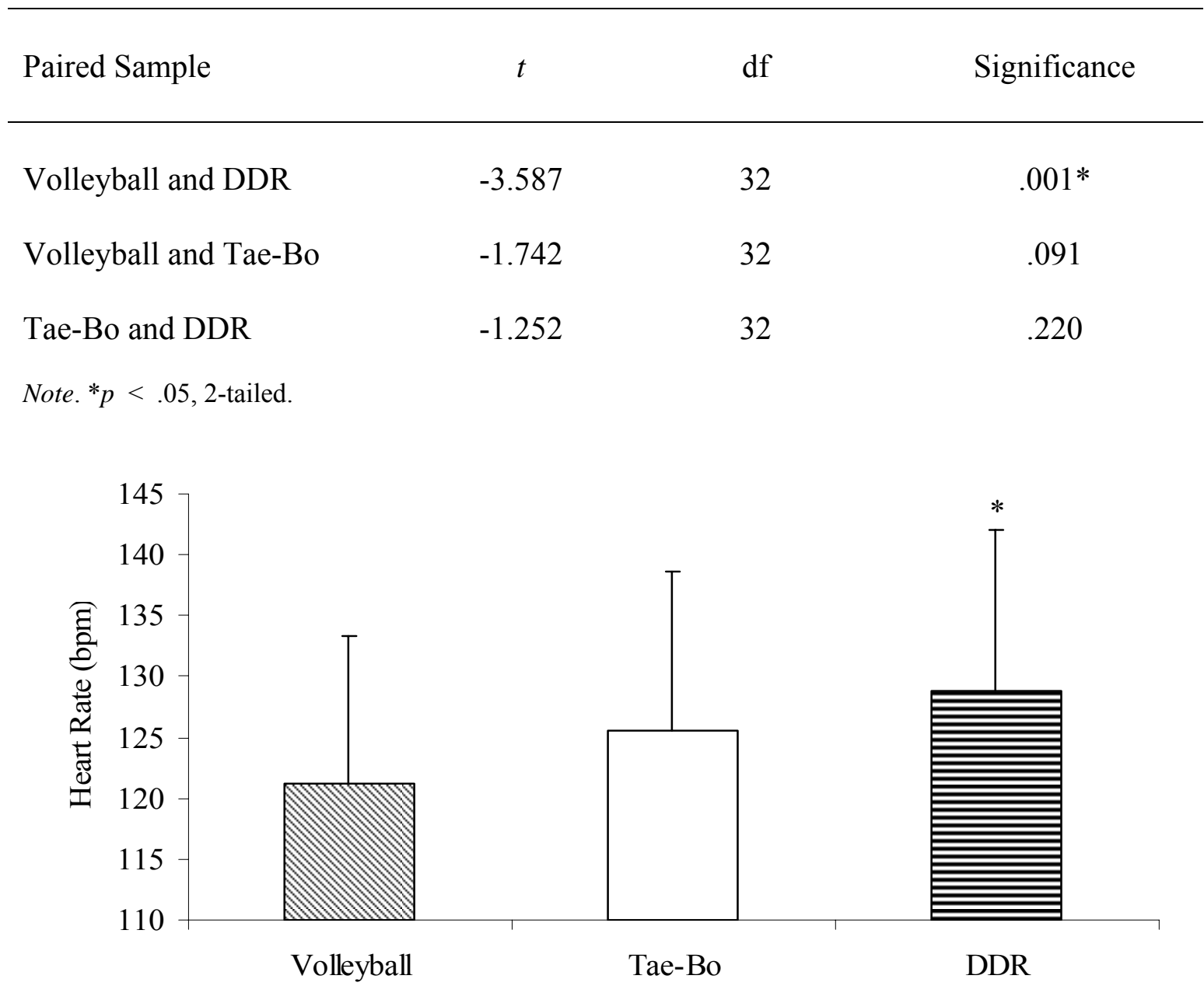

Figure 5. Mean heart rate (+standard deviation) for all participants in volleyball, Tae-Bo and DDR. ${ }^{*} p<.05$, DDR significantly higher than volleyball.

Between-subject comparisons using repeated measures ANOVA (split plot factorial 222.3) were also performed to determine significant differences between selected groups of participants. The dependent variable was heart rate. The independent variable was activity type (three levels), and participants were blocked according to 
gender, grade level, and Body Mass Index (BMI). Each block had two groups; gender had male and female, grade level had fifth-grade and sixth-grade, and BMI had $<85^{\text {th }}$ percentile and $\geq 85^{\text {th }}$ percentile. Group was the other independent variable. Group means and standard deviations for heart rate are presented in Appendix O. Main effects for gender, $F(1,25)=.976, p>.05$, and grade level, $F(1,25)=.609, p>.05$, were not significant. BMI, however, revealed a significant main effect, $F(1,25)=4.757, p<.05$, which suggested that heart rates were significantly different between subjects grouped by BMI for one or more activity types. Subsequent post-hoc independent $t$ tests were performed to determine which activity types showed significantly different heart rates among BMI groups. The results are presented graphically in Figure 6. Tae-Bo heart rates did not differ significantly according to BMI category, as the $t$ test result was $t(31)=-$ $.794, p>.05$. Both DDR and volleyball did differ significantly. Volleyball had a $t$ test result of $t(31)=-2.554, p<.05$, indicating that the heart rates for those participants in the upper BMI category ( $\geq 85^{\text {th }}$ percentile) had significantly higher heart rates than those in the lower BMI category $\left(<85^{\text {th }}\right.$ percentile) during volleyball. DDR had a $t$ test result of $t(31)=-2.492, p<.05$, indicating that the heart rates for those participants in the upper BMI category had significantly higher heart rates than those in the lower BMI category during DDR. 


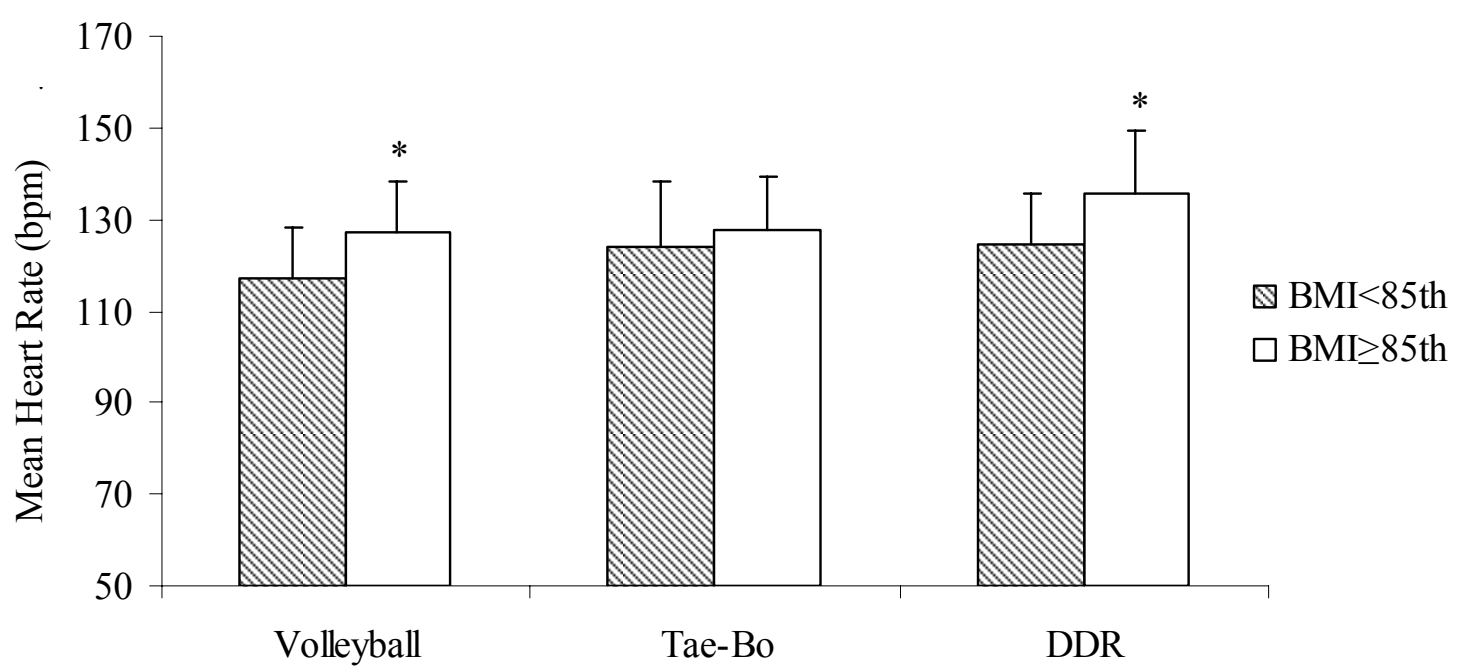

Figure 6. Mean heart rate (+ standard deviation) for two BMI groups for volleyball, TaeBo and DDR. ${ }^{*} p<.05, \mathrm{BMI} \geq 85^{\text {th }}$ percentile significantly different from $\mathrm{BMI}<85^{\text {th }}$ percentile.

\section{Step Count}

Total step count was recorded for each trial using the Walk4Life pedometer. Step count data including means and standard deviations are presented in Appendix N. A 3 x 6 repeated measures within subjects analysis of variance was conducted in the following manner; the independent variables were activity type (3 levels) crossed with trials (6 levels). The dependent variable was step count. This analysis yielded a significant main effect for activity type, $F(2,64)=38.319, p<.05$, and a significant main effect for trials, $F(5,160)=4.388, p<.05$. Multiple comparisons for the main effect for activity type are presented later in this section. A significant interaction between activity type and trials was also found, $F(10,320)=5.557, p<.05$. This indicated that the pattern of change of step count across trials was significantly different among activity types. Figure 7 shows 
the trends in step count over each trial for all activity types. Subsequent multiple comparisons for the interaction revealed that with the exception of Trial 1, DDR differed significantly from Tae-Bo in step count across trials $(p<.05)$. DDR and volleyball step counts revealed significant differences across all six trials $(p<.05)$. As shown in Figure 7, a significant difference in step count between Trial 1 and Trial 6 was found for DDR, with $t(32)=-2.219, p<.05$. Step count increased over trials for DDR. At Trial 4, volleyball step count was significantly lower than that of Tae-Bo, with $t(32)=-6.275, p<$ .05 .

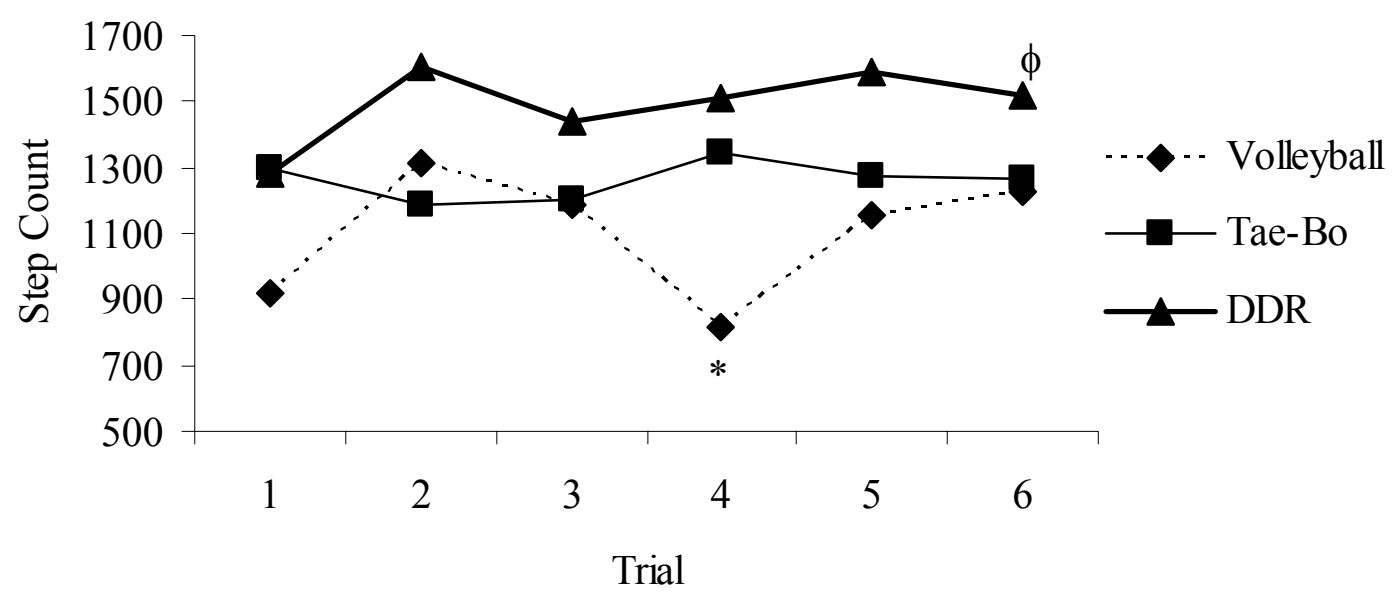

Figure 7. Mean step count per trial for all participants. ${ }^{*} p<.05$, volleyball significantly lower than Tae-Bo; ${ }^{\phi} p<.05$, Trial 6 significantly higher than Trial 1 for DDR.

The overall mean step counts for each activity type were calculated, and were as follows (with standard deviations in parentheses): volleyball, 1,104.18 (313.32); Tae-Bo, 1,262.51 (343.41); and DDR, 1,490.71 (322.30). Subsequent multiple comparisons for 
the main effect revealed significant differences, as shown in Table 13. Overall mean step counts for each activity type are presented in Figure 8.

Table 13

Multiple Comparisons for the Significant Main Effect of Activity Type on Step Count

$\begin{array}{llll}\text { Paired Samples } & t & \mathrm{df} & \text { Significance }\end{array}$

$\begin{array}{llll}\text { Volleyball and DDR } & -7.979 & 32 & .00^{*}\end{array}$

$\begin{array}{llll}\text { Volleyball and Tae-Bo } & -3.449 & 32 & .002 *\end{array}$

$\begin{array}{llll}\text { Tae-Bo and DDR } & -5.977 & 32 & .00^{*}\end{array}$

Note. ${ }^{*} p<.05,2$-tailed.

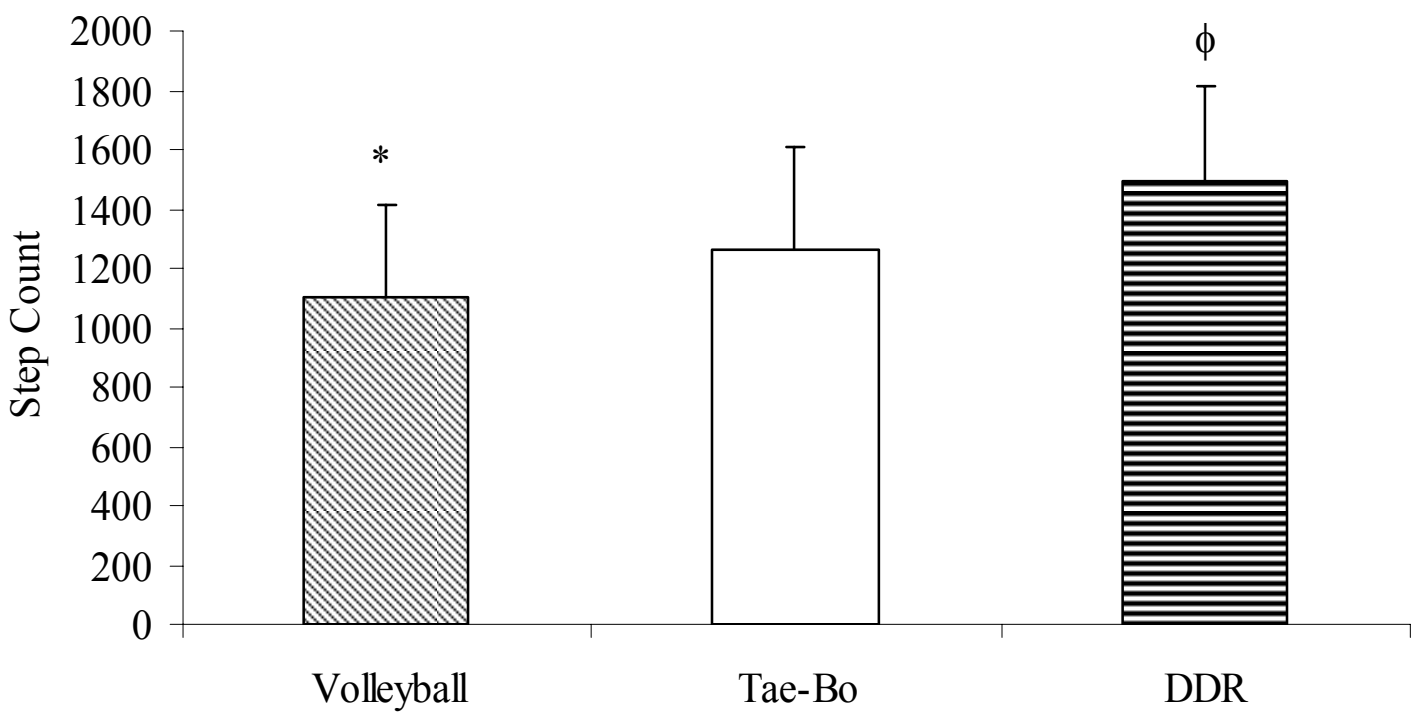

Figure 8. Mean step count (+standard deviation) for each activity type for all participants. ${ }^{*} p<.05$, volleyball significantly lower than Tae-Bo and DDR. ${ }^{\phi} p<.05$, DDR significantly higher than Tae-Bo. 
Additionally, steps per minute were calculated based upon total elapsed time for each physical education lesson. The mean steps per minute were as follows (with standard deviations in parentheses); volleyball, 42.48 (12.31); Tae-Bo 58.64 (15.84); and DDR, 61.07 (13.29). Based on a paired samples $t$ test, Tae-Bo and DDR did not demonstrate a significance difference from one another, $t(32)=-1.297, p<.05$. Both Tae-Bo and DDR were significantly different from volleyball; $t(32)=-7.483, p<.05$ and $t(32)=-8.849, p<.05$, respectively

Between-subjects comparisons using repeated measures ANOVA (split plot factorial 222.3) were also performed to determine significant differences between selected groups of participants. The independent variable was activity type (3 levels), and participants were blocked according to gender, grade level, and Body Mass Index (BMI), thus making group the other independent variable. Each block had two groups; gender had male and female, grade level had fifth-grade and sixth-grade, and BMI had $<85^{\text {th }}$ percentile and $\geq 85^{\text {th }}$ percentile. The dependent variable was step count. Group means and standard deviations for step count are presented in Appendix O. Main effects for gender, $F(1,25)=.021, p>.05 ;$ Grade Level, $F(1,25)=1.496, p>.05 ;$ and BMI, $F(1,25)=$ $.331, p>.05$, did not yield significant results. Step count in each of the three activity types was not influenced by BMI, grade level, or gender in this sample.

\section{Active Time}

Total elapsed time for each session (trial) was measured with the Cateye heart rate monitor. Exercise sessions averaged 24 minutes in length. Tae-Bo had the lowest overall mean elapsed time of 22 minutes, followed by DDR at 25 minutes, and volleyball at 26 
minutes. Active time was measured using the Walk4Life pedometer. Both instruments were set at the same time prior to the beginning of the physical education class. Tae-Bo elicited the highest percent active time (70.06\%), followed closely by DDR (68.50\%), then lastly, by volleyball $(54.36 \%)$. Percent active time data across trials are presented in Appendix N.

A $3 \times 6$ repeated measures within-subjects analysis of variance was conducted in the following manner. The independent variables were activity type ( 3 levels) crossed with trials (6 levels), and the dependent variable was percent active time. This analysis revealed a significant main effect for activity type, $F(2,64)=53.925, p<.05$, and a significant main effect for trial, $F(5,160)=4.048, p<.05$. A significant interaction was found between activity type and trial, $F(10,320)=4.134, p<.05$. This result indicated that the pattern of change in percent active time across trials was significantly different among activity types. Figure 9 displays the percent active time trends across trials for each of the activity types. Subsequent paired samples $t$ tests were conducted to determine significant differences among means. The results were as follows: a significant difference in percent active time was found between volleyball and DDR across all trials $(p<.05)$. Tae-Bo and DDR differed significantly only at Trial $4(p<.05)$. 


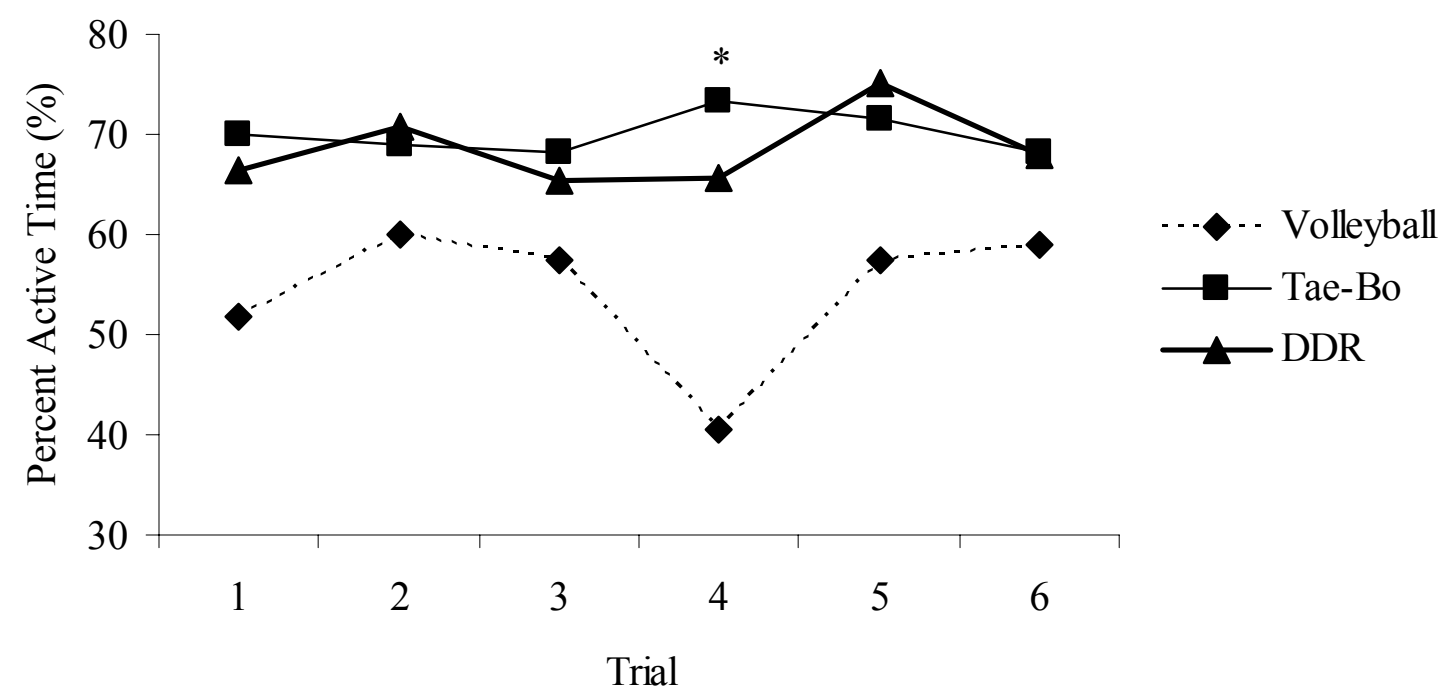

Figure 9. Percent active time per trial for all participants in volleyball, Tae-Bo and DDR. $* p<.05$, Tae-Bo significantly higher than DDR at Trial 4.

When collapsed across trials, overall means for percent active time were determined. Mean percent active times (with standard deviations in parentheses) for volleyball, Tae-Bo, and DDR were 54.36 (13.03), 70.06 (12.62), and 68.50 (10.0), respectively. Subsequent multiple comparisons for the main effect of activity type revealed the following results. Volleyball was significantly different from both Tae-Bo and DDR. Tae-Bo and DDR did not differ significantly. These results are presented in Table 14. Figure 10 graphically displays the means and standard deviations of percent active time for each activity type, as well as significant $t$ test results. 
Table 14

Multiple Comparisons for the Significant Main Effect of Activity Type on Percent Active

Time

Paired Samples

$t$

df

Significance

Volleyball and DDR

8.684

32

$.00^{*}$

Volleyball and Tae-Bo

8.595

32

$.00 *$

Tae-Bo and DDR

$-1.023$

32

Note. ${ }^{*} p<.05,2$-tailed.

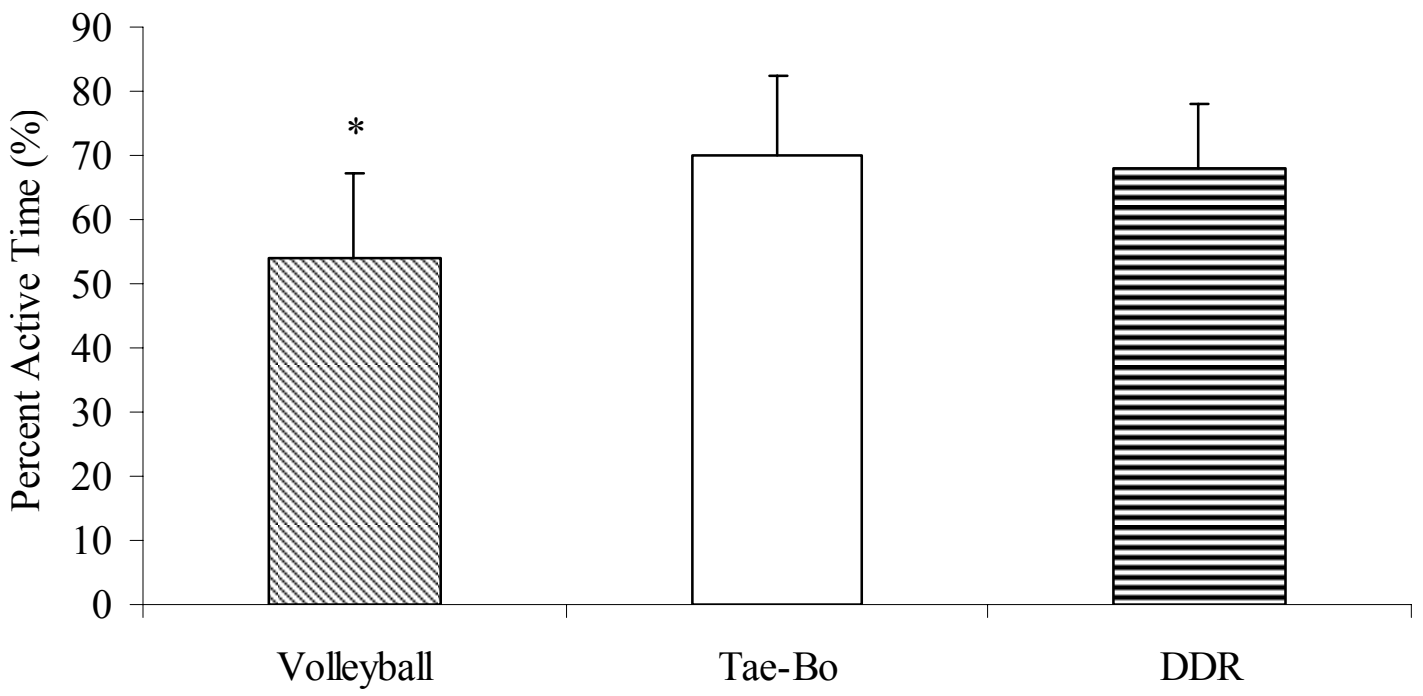

Figure 10. Percent active time (+standard deviation) for each activity type; ${ }^{*} p<.05$, volleyball significantly lower than Tae-Bo and DDR.

Between-subjects comparisons using repeated measures ANOVA (split plot factorial 222.3) were also performed to determine significant differences between 
selected groups of participants. The independent variable was activity type (3 levels), and participants were blocked according to gender, grade level, and BMI. Each block had two groups; gender had male and female, grade level had fifth-grade and sixth-grade, and BMI had $<85^{\text {th }}$ percentile and $\geq 85^{\text {th }}$ percentile. Group was the second independent variable. The dependent variable was percent active time. Group means and standard deviations are presented in Appendix O. Main effects for gender, $F(1,25)=.002, p>.05$, grade level, $F(1,25)=3.808, p>.05$, and BMI, $F(1,25)=.451, p>.05$, were not significant. Percent active time in each of the three activity types was not affected by BMI, grade level, or gender in this sample.

Pearson product-moment correlation coefficients were calculated between step count and active time for each activity type. Step count was positively correlated with active time for all three activity types. The correlation coefficients were as follows; volleyball, $r=.978$; Tae-Bo, $r=.935$; and DDR, $r=.833(p<.05)$.

\section{Perceived Exertion}

Perceived exertion rates were self reported after each exercise session for all participants. Scores on a scale of 0-10 were recorded for each participant immediately following each trial. Means and standard deviations for rates of perceived exertion across each trial are presented in Appendix N. A 3 x 6 repeated measures within-subjects analysis of variance was conducted in the following manner; the independent variables were activity type (3 levels) crossed with trials (6 levels), and the dependent variable was rate of perceived exertion (RPE). The analysis revealed a main effect for activity type, $F(2,64)=22.554, p<.05$, but not for trials, $F(5,160)=2.241, p>.05$. A significant 
interaction was found between activity type and trials, $F(10,320)=2.673, p<.05$. This indicated a significant difference in the pattern of RPE across trials among each of the three activities. Figure 11 displays the trends across trials for each activity type. Multiple comparisons for the significant interaction revealed that volleyball and DDR differed significantly only at Trials 2,4 , and $5(p<.05)$, and Tae-Bo and DDR differed significantly on all trials except Trial $5(p<.05)$.

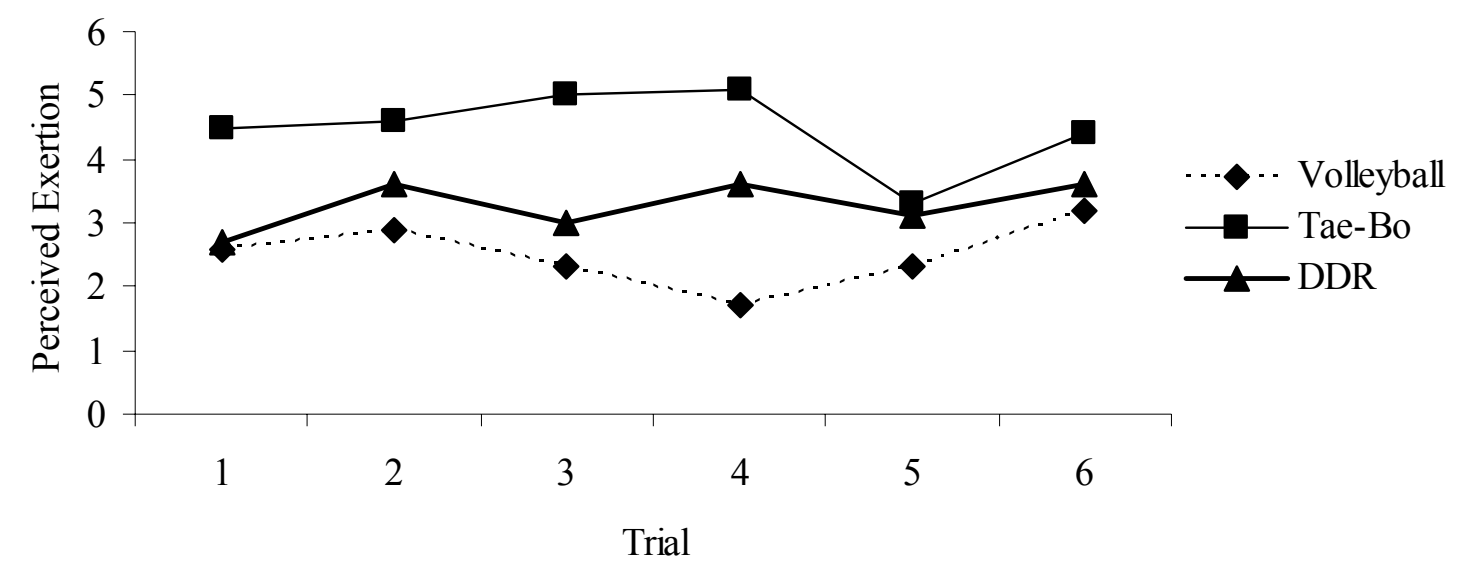

Figure 11. Mean rate of perceived exertion per trial for all participants. Tae-Bo was significantly higher than DDR except at Trial $5(p<.05)$.

The overall mean rates of perceived exertion when trials were collapsed for each activity type were determined. These means were as follows (with standard deviations in parentheses); volleyball, 2.50 (2.35); DDR, 3.26 (2.58); and Tae-Bo, 4.46 (2.92). Subsequent multiple comparisons on the main effect for activity type revealed significant differences among all activity types. These results are displayed in Table 15. Figure 12 displays the means and standard deviations of rate of perceived exertion in this sample. 
Table 15

Multiple Comparisons for the Significant Main Effect of Activity Type on Rate of Perceived Exertion

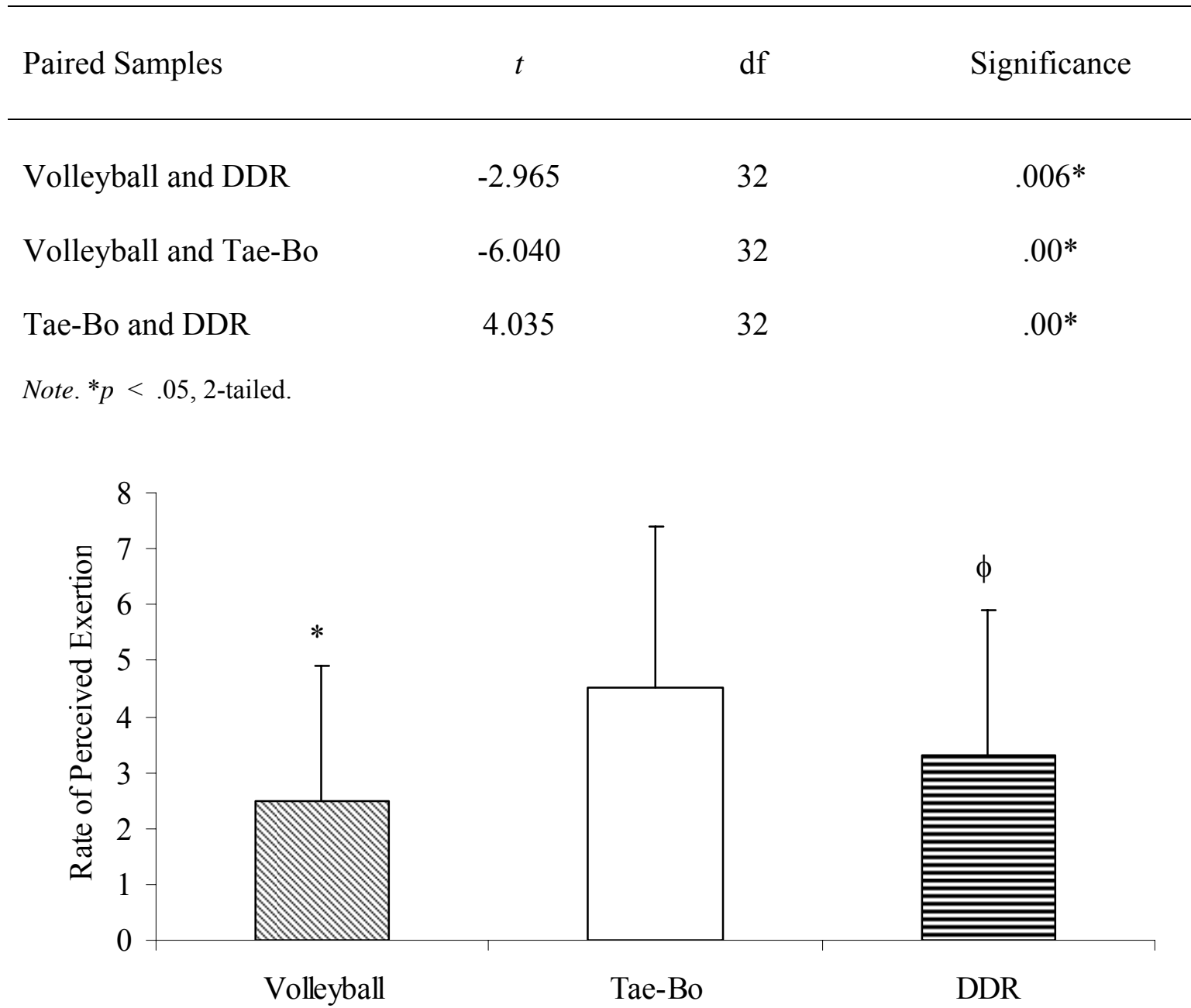

Figure 12. Mean rate of perceived exertion for volleyball, Tae-Bo and DDR. * $p<.05$, volleyball significantly lower than Tae-Bo and DDR. ${ }^{\phi} p<.05$, DDR significantly lower than Tae-Bo. 
Between-subjects comparisons using repeated measures ANOVA (split plot factorial 222.3) were also performed to determine significant differences among selected groups of participants. The independent variable was activity type (3 levels), and participants were blocked according to gender, grade level, and BMI. Each block had two groups; gender had male and female, grade level had fifth-grade and sixth-grade, and BMI had $<85^{\text {th }}$ percentile and $\geq 85^{\text {th }}$ percentile. Group was the other independent variable. The dependent variable was RPE. Group means and standard deviations for rate of perceived exertion are presented in Appendix $O$. Main effects for gender, $F(1,25)=$ $.015, p>.05$, grade level, $F(1,25)=3.885, p>.05$, and BMI, $F(1,25)=.364, p>.05$, were not significant. Rates of perceived exertion were not influenced by BMI, grade level, or gender.

\section{Heart Rate and Perceived Exertion}

The trends for heart rate and perceived exertion over six trials show contrasting results. The rate of perceived exertion is consistently higher for Tae-Bo than the other two activity types, with some significant differences as noted in previous sections. However, heart rates for Tae-Bo do not show a similar trend. DDR heart rates are consistently higher than Tae-Bo, particularly over the first four trials. DDR heart rate was significantly higher than Tae-Bo at Trial 2. Figure 13 shows the mean heart rates and rates of perceived exertion for each trial for each of the three activity types. It should be noted that DDR heart rates, though higher than Tae-Bo, tend to cluster at a lower rate of perceived exertion. Similarly, Tae-Bo heart rates cluster toward the middle of the three activity types, but at a higher rate of perceived exertion. Volleyball heart rates cluster 
together at the lower end of the range, and with concomitant lower rates of perceived exertion.

Pearson product-moment correlation coefficients were calculated for these two variables for all three activity types, but none was found to be significant. Correlation coefficients are reported in Appendix P.

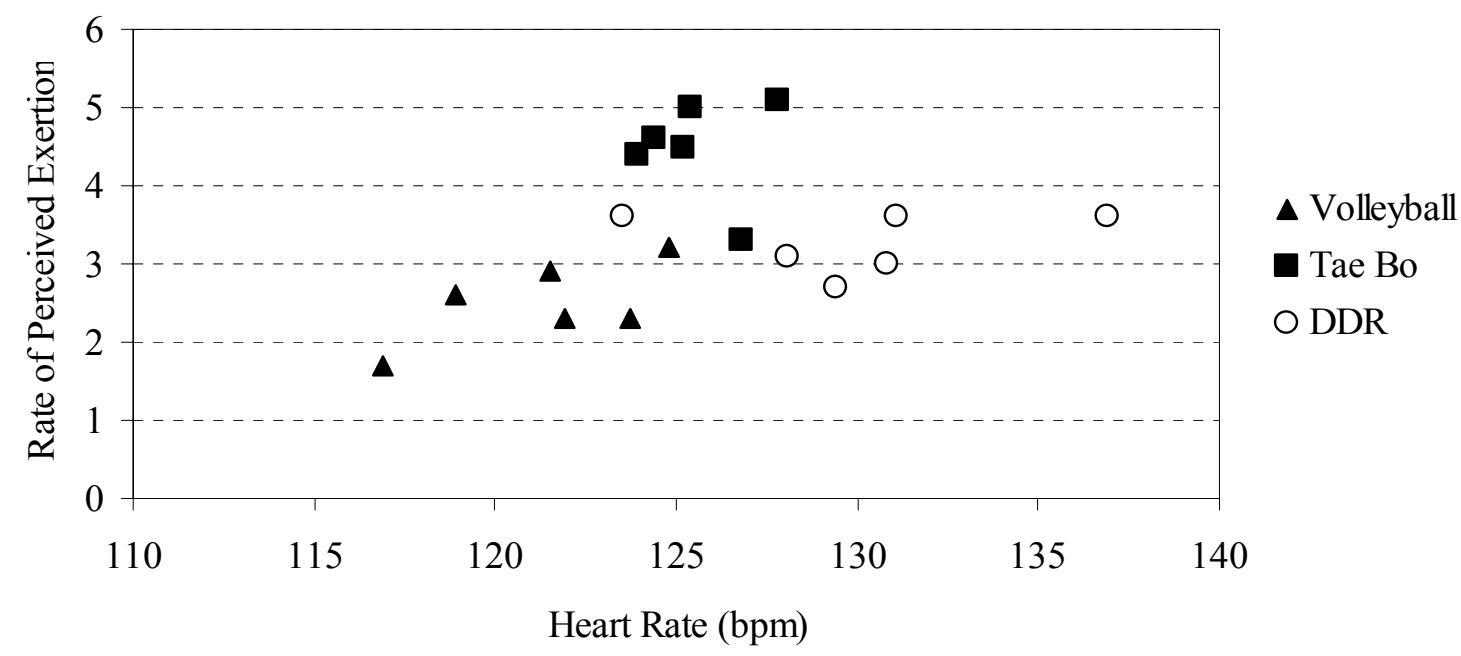

Figure 13. Scatter diagram of mean scores per trial for heart rate versus rate of perceived exertion.

Figure 14 displays the same data as Figure 13, but this time, mean rates of perceived exertion are layered on top of mean heart rates for each activity type. This representation demonstrates the differences between activity types for these two variables. 


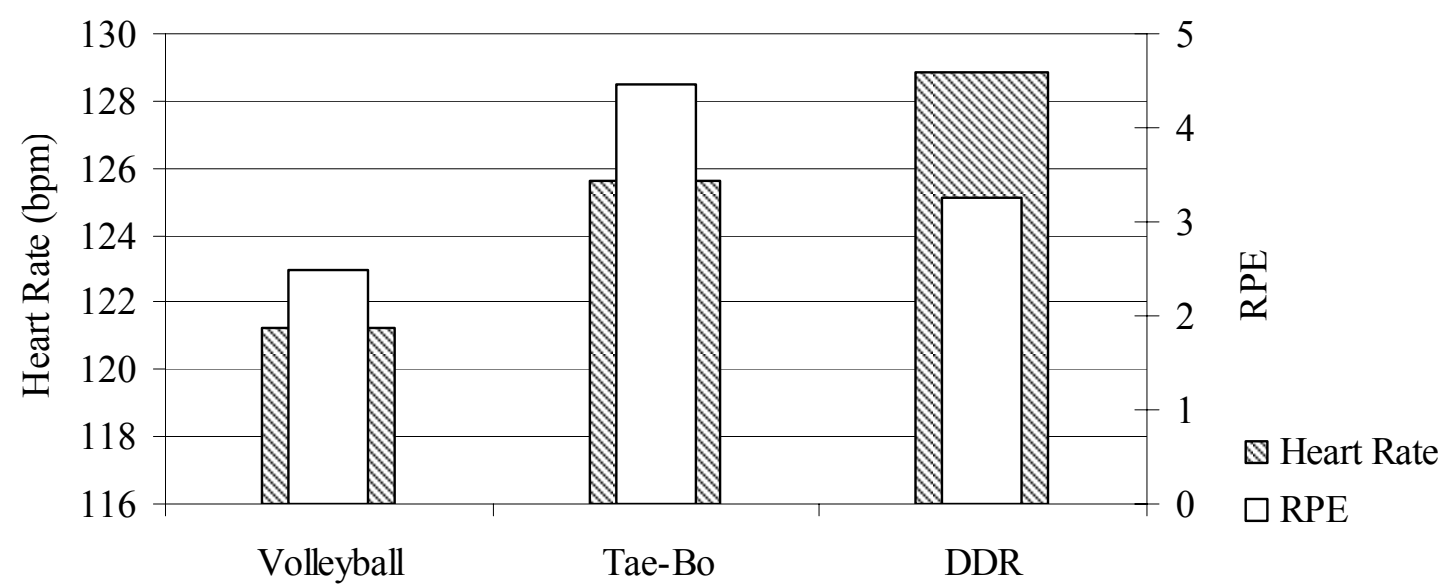

Figure 14. Mean heart rate and mean RPE for volleyball, Tae-Bo and DDR.

\section{Enjoyment}

Enjoyment scores were gathered at the conclusion of each activity session. The self-report questionnaire was scored on a seven-item Likert scale from 1-5, so the highest possible score was 35 . Mean enjoyment scores and standard deviations for each trial are presented in Appendix N. A $3 \times 6$ repeated measures within subjects analysis of variance was conducted in the following manner; the independent variables were activity type (3 levels) crossed with trial (6 levels), and the dependent variable was enjoyment score. Results indicated a main effect for activity type, $F(2,64)=29.356, p<.05$, but no main effect for trials, $F(5,160)=2.191, p>.05$. Multiple comparisons for the main effect for activity type are presented later in this section. There was a significant interaction between activity type and trials, $F(10,320)=2.616, p<.05$. The pattern of enjoyment change across trials was significantly different among activity types, as displayed in Figure 15. Multiple comparisons for the interaction revealed that DDR enjoyment was significantly higher than that of volleyball only at Trials 1 and $2(p<.05)$, and DDR 
enjoyment was significantly higher than that of Tae-Bo across all trials $(p<.05)$.

Enjoyment scores for Tae-Bo showed a significant decrease from Trial 1 to Trial 6, $t(32)=2.503, p<.05$.

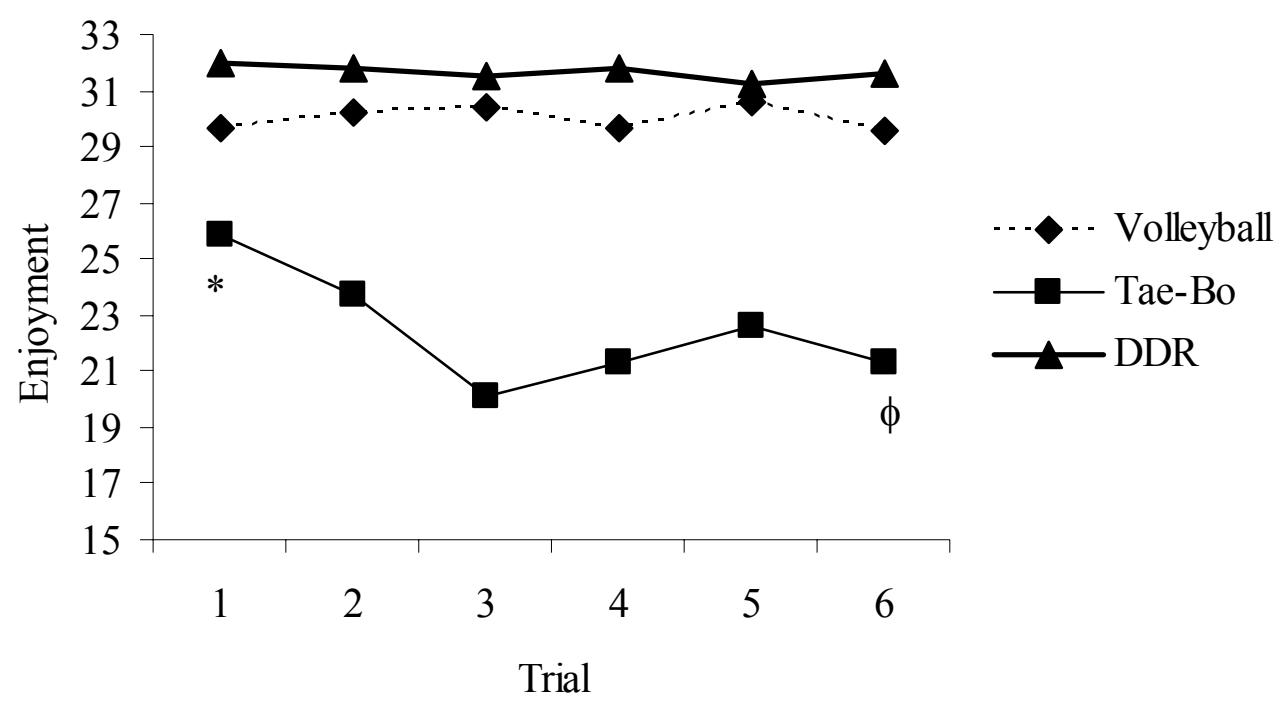

Figure 15. Mean enjoyment score per trial for all participants. ${ }^{*} p<.05$, Tae-Bo score significantly less than DDR score for all trials. ${ }^{\phi} p<.05$, Trial 6 significantly lower than Trial 1.

When enjoyment scores were collapsed across trials, an overall mean for each activity type was determined. Mean enjoyment scores (with standard deviations in parentheses) for volleyball, Tae-Bo, and DDR were 30.01 (6.04), 22.46 (8.69), and 31.65 (5.95), respectively. Table 16 displays the results of the multiple comparisons for the main effect for activity type. Figure 16 depicts the differences in overall means for enjoyment scores across the three activity types. Significant differences were noted among all activity types. 
Table 16

Multiple Comparisons for the Significant Main Effect of Activity Type on Enjoyment Score

Paired Samples

$t$

df

Significance

Volleyball and DDR

$-2.262$

32

$.031 *$

Volleyball and Tae-Bo

5.012

32

$.00 *$

Tae-Bo and DDR

$-6.319$

32

$.00 *$

Note. ${ }^{*} p<.05$, 2-tailed.

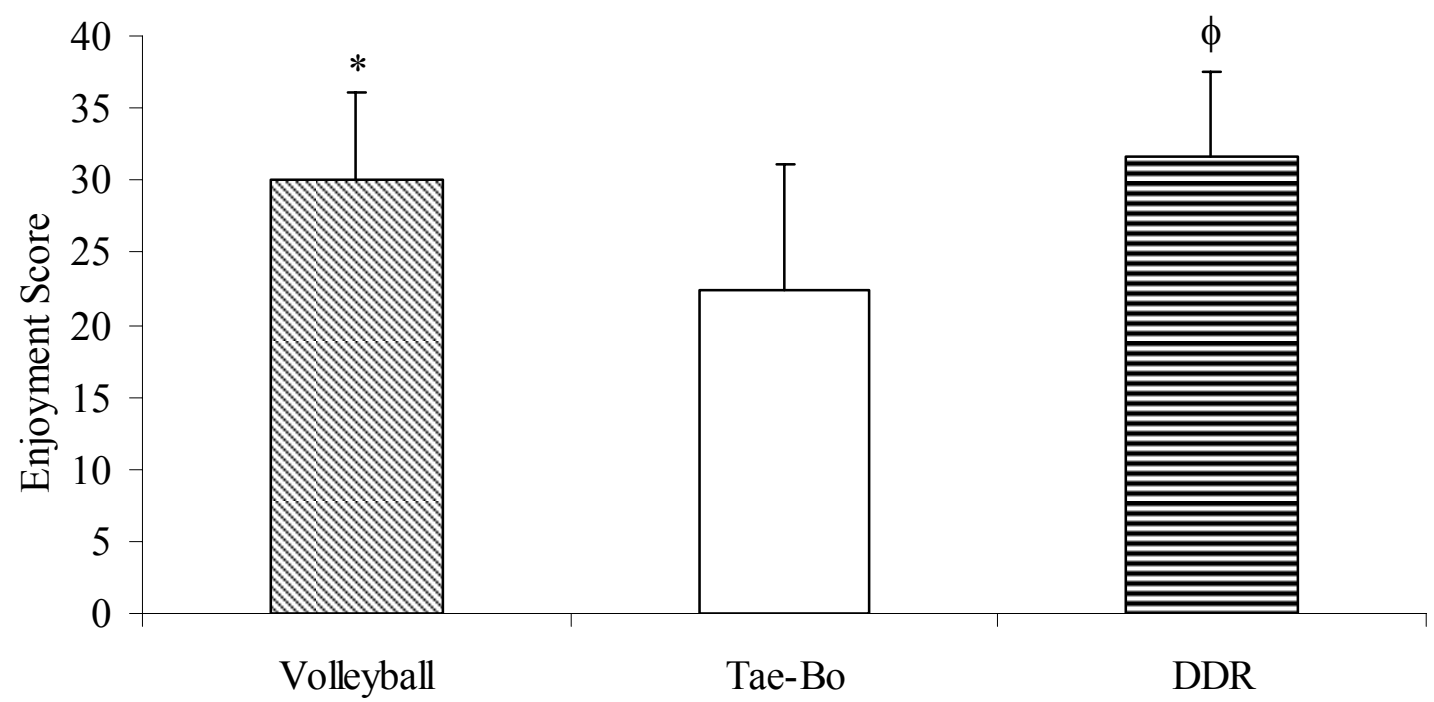

Figure 16. Mean enjoyment score for volleyball, Tae-Bo, and DDR. * $p<.05$, volleyball significantly higher than Tae-Bo. ${ }^{\phi} p<.05$, DDR significantly higher than Tae-Bo and volleyball. 
Between-subjects comparisons using repeated measures ANOVA (split plot factorial 222.3) were also performed to determine significant differences between selected groups of participants. The independent variable was activity type (3 levels), and participants were blocked according to gender, grade level, and BMI. Each block had two groups; gender had male and female, grade level had fifth-grade and sixth-grade, and BMI had $<85^{\text {th }}$ percentile and $\geq 85^{\text {th }}$ percentile. Group served as the second independent variable. Group means and standard deviations for enjoyment scores are presented in Appendix O. Main effects for gender, $F(1,25)=.230, p>.05$; grade level, $F(1,25)=$ $.842, p>.05$; and BMI, $F(1,25)=.980, p>.05$ were not significant. Enjoyment score was not influenced by BMI, grade level, or gender.

A paired sample Pearson product-moment correlation coefficient was calculated between enjoyment score and rate of perceived exertion across all three activity types. Not one correlation was found to be significant. The correlation coefficient for these two variables for Tae-Bo approached significance in a negative direction $(r=-.302, p>.05)$. Similarly, correlation coefficients were calculated between enjoyment scores and elapsed time. The correlation for DDR was significant, $r=-.345, p<.05$. All correlation coefficients are presented in Appendix P.

\section{Self-Efficacy}

Self-efficacy was measured twice during the intervention; prior to the beginning of the intervention, and once again at the conclusion of the intervention. Self-efficacy scores were calculated for each participant pre- and post-intervention based on the eightitem questionnaire presented in Appendix K. The highest possible score was 40 based on 
a 1-5 Likert scale. The mean scores for pre- and post-intervention were calculated and subjected to a paired samples $t$ test. Paired $t$ testing revealed that self-efficacy did not change significantly over the course of the intervention. Self- efficacy data are presented in Table 17. Responses to individual items on the post-intervention self-efficacy questionnaire are presented in Table 18.

Table 17

Mean Self-efficacy Scores and Subsequent t test Results for Fifth- and Sixth-Grade Participants

\begin{tabular}{ccccc}
\multicolumn{5}{c}{ Self-Efficacy $(\mathrm{N}=33)$} \\
\hline Pre-Intervention & Post-Intervention & $\mathrm{t}$ & $\mathrm{df}$ & Significance \\
$(\mathrm{Mean} \pm \mathrm{SD})$ & $($ Mean $\pm \mathrm{SD})$ & & & \\
\hline $31.15 \pm 5.60$ & $31.97 \pm 6.93$ & -0.963 & 32 & $0.343 *$ \\
Note. $* p>.05$ & & & &
\end{tabular}


Table 18

Percent of Responses to Self-Efficacy Questionnaire Items Post-Intervention (N=33)

\begin{tabular}{lccccc}
\hline & Disagree & Disagree & & Agree a & Agree a \\
I can be physically active... & a lot & a little & Neither & little & lot \\
\hline during my free time on most & & & & & \\
days & 6.1 & 3 & 6.1 & 18.2 & 66.7 \\
I can ask a Parent or adult & 9.1 & 3 & 15.2 & 33.3 & 39.4 \\
even if I could watch TV/Video & 18.2 & 0 & 9.1 & 24.2 & 48.5 \\
even if it's hot/cold outside & 3 & 6.1 & 12.1 & 18.2 & 60.6 \\
I can ask my best friend & 3 & 6.1 & 6.1 & 18.2 & 66.7 \\
even if I have a lot of homework & 21.2 & 9.1 & 6.1 & 24.2 & 39.4 \\
I have the coordination & 12.1 & 0 & 6.1 & 24.2 & 57.6 \\
no matter how busy I am & 21.2 & 9.1 & 9.1 & 18.2 & 42.4
\end{tabular}




\section{CHAPTER 5}

\section{DISCUSSION}

The overall goal of this study was to explore the viability of DDR as a means to actively engage elementary school children in physical education. The results clearly show that DDR has the potential to impact physical education classes in a positive way. This may have significant implications for teachers making curricular decisions for their physical education classes, and perhaps more importantly, enhance physical activity levels of youth. DDR demonstrated significant relationships with each of the following dependent variables: heart rate, step count, perceived exertion, enjoyment, and active time. DDR elicited the highest exercise heart rates, the highest mean step counts, and the highest enjoyment levels when compared to volleyball and Tae-Bo. Conversely, DDR did not elicit the highest rates of perceived exertion. Therefore, participants involved in DDR exercised vigorously without realizing it. The following sections will discuss each of the research hypotheses in light of the results presented in the previous chapter. The implications, limitations and future directions of this research will also be addressed.

\section{Demographics}

The group of participants selected for this study reported that they were more active than the statistics presented by the CDC (2003). The CDC found that $61.5 \%$ of children aged 9-13 did not participate in any physical activity during non-school hours. This was certainly not the case in the present study. Only 3\% (one out of 33 respondents) of study participants reported no physical activity whatsoever during non-school hours on weekdays or weekends. Though levels of physical activity during non-school hours did not show a significant change during the intervention, they were self-reported and 
therefore possibly over-estimated from the outset. Thus, caution must be used when interpreting the results. It does appear, however, that this sample of fifth- and sixth-grade participants was relatively active, similar to the findings of Guerra et al. (2003) and Sallis and Patrick (1994). As noted in Table 10, nearly half the participants reported that they were active for more than 60 minutes on weekdays (15 out of 33) and weekends (16 out of 33) post-intervention. The trend for physical activity increased, but it did not reach significance. NASPE (2004) recommends that children accumulate a minimum of 60 minutes per day of moderate-to-vigorous physical activity on most days of the week. Physical activity time reported in this study did not include physical education class, which was provided three to four times per week for 30 minutes, so levels of physical activity reported may be less than that actually accumulated. It is possible that participants did not fully understand the definition of moderate-to-vigorous physical activity, and therefore included activities of low intensity like walking in their estimations. In the future, a more detailed physical activity questionnaire may elicit more accurate responses. The use of sealed pedometers or accelerometers for 24-hour periods would provide more concrete data regarding physical activity patterns in this population. While this group reported that it was particularly active, it may not necessarily represent other children of the same age in other regions of North America. In Ontario, there is great variability in the number of physical education classes provided to students each week, so one school may be quite different from another school. Seasonal variations in climate may also have impacted these results, which were collected during the winter months. For example, participants might have been less active during the winter months since they would choose not to engage in outdoor activities. There are certainly regional 
variations in climate that affect outdoor activity opportunities. Other factors that reduce generalizability to other populations include the extracurricular opportunities provided within schools, rural versus urban schools (e.g. students walking to school versus those who are bussed), economic issues for parents, and community programming for children.

Measures of body mass index do not necessarily support the physical activity results, however. In this sample, $12 \%$ of participants were at risk of overweight, and $27 \%$ were overweight according to the CDC (2001) guidelines. This proportion is more than that stated by the CDC, which, based on the National Center for Health Statistics (2005), reports $16 \%$ of children (ages 6-11) and adolescents (ages 12-19) were overweight. Those at risk of overweight were not reported by the CDC statistics. Steinbeck (2001) noted that physical inactivity increases the risk of obesity. Since body mass index is a valid measure of obesity in children (Pietrobelli et al., 1998), one could conclude one of two things: this group of children have poor diets and consume excess calories, or they are insufficiently active to maintain healthy body weights. While it is likely a combination of these two factors, the results of this study cannot conclusively explain the high rates of overweight and obesity in this population. Another factor that might have overestimated BMI levels in this population may be related to the clothing that participants were wearing at the time of measurement. The study was conducted during the winter months so heavier clothing, and perhaps clothing that became wet during recess, might have increased BMI results (even though shoes had been removed).

Additionally, further examination of the data revealed that those students who were at risk of overweight $\left(\geq 85^{\text {th }}\right.$ and $<95^{\text {th }}$ percentile) or overweight $\left(\geq 95^{\text {th }}\right.$ percentile) consistently reported higher physical activity levels than those students at a healthy 
weight $\left(\geq 5^{\text {th }}\right.$ and $<85^{\text {th }}$ percentile $)$ or underweight $\left(<5^{\text {th }}\right.$ percentile $)$. These differences were not significant, but this may have been an attempt on the part of the overweight participants to appear more active than they actually were. In future investigations, more careful monitoring and collection of physical activity data prior to any intervention would be relevant information, particularly to determine changes in physical activity patterns as a result of that intervention.

\section{Heart Rate}

It is very interesting to note that DDR elicited the highest heart rates of the three activity types. This becomes even more remarkable when compared to Tae-Bo, which is an activity challenging participants to be continually active as they follow the leader on the television monitor. Typical to most commercially available exercise videos, Tae-Bo requires constant movements of varying intensities stressing different components of health-related fitness. Though heart rates would fluctuate depending on the phase of the workout (e.g. warm up, muscular endurance, cardiovascular endurance, etc.), participants constantly move in rhythm to the music, following the actions of the workout leader. As the researcher observed these sessions, participants were continually active, but the intensity of those movements generally decreased as the workout progressed. This was likely a function of fitness level as well as enjoyment. Based on the observations of the researcher, students tired very quickly, particularly those who started energetically. They did respond to teacher prompting, but they were not able to sustain the intensity levels indicated on the video tape. The trend line for Tae-Bo heart rate over the six trials remained relatively flat, indicating similar exercise intensity for each trial. There may be several possible explanations. Teachers did their best to encourage their students to keep 
participating, and this task became more challenging with each trial, as students enjoyed Tae-Bo less and less over time. Therefore, the successful encouragement of teachers might be one explanation; students were responsive to teacher prompting and did their best to maintain their participation. A second explanation may point to the fact that TaeBo involved both arm and leg movements, separately and in combination. Many participants had difficulty coordinating their own bodily movements to correspond to those of the leader. Even half-hearted attempts to move both arms and legs (as opposed to legs only) would boost heart rates. There has been little research exploring the effect of exercise videos on heart rate in children, so it is difficult to support the finding of this study. However, NASPE (2004) guidelines suggest children should participate in several bouts of physical activity lasting 15 minutes or more each day. Based on the results of this study, Tae-Bo fits under this guideline as an appropriate activity. In looking at the enjoyment results, in which Tae-Bo is significantly lower than the other two activities, teachers should use caution when selecting activities like Tae-Bo for their students if their goal is to enhance physically active lifestyles and encourage lifelong physical activity.

One significant between-subjects effect with respect to heart rate involved body mass index. For both volleyball and DDR, heart rates were significantly higher for those participants who were at risk of overweight or overweight. There was no significant difference in heart rate between the two groups during Tae-Bo. This interesting finding may suggest one possible mechanism. Because volleyball and DDR are activities that are intermittent in nature, active periods are interspersed with non-active periods. It is proposed that for those participants who were in the higher BMI category, rest periods 
were not sufficiently long enough to permit their heart rates to drop before they once again began an active period. It may be that these participants had lower fitness levels, and/or cardiovascular efficiency may not have been as high as it was in those participants in the lower BMI category. Recovery from exercise was not within the scope of this study; however, opportunities to further explore the relationship between BMI and heart rate would be an interesting area of future research.

In comparing the heart rate results to those previously reported DDR compared favorably as a moderate-to-vigorous physical activity. Kelly (2000) reported that heart rates in the range of 123-139 beats per minute would be categorized as moderatevigorous physical activity for 9-10 year old children. Heart rates for DDR in the present study ranged from 100-166 beats per minutes with an average of 129 beats per minute. In a recent DDR study, Yang and Graham (2005) reported heart rates ranging from 113-178 with a mean of 155 beats per minute. The age group of this sample was $12-13$ years, slightly older than the current sample. Age may be one explanation for the different findings. Gilbert (2005) suggested that since heart rates in children 5-12 years are age independent, it may be problematic to compare heart rates among children. The motor ability of an older group, even one or two years, likely made a difference in how quickly the participants were able to learn the game and play it competently. It is not indicated how much experience the participants had with DDR in the Yang and Graham study, but the elevated heart rates during only one 45 minute DDR session may take into account the extra effort required to learn the game. The inefficiency of movement was reflected in Trial 2 in the present study, which had a mean heart rate of 140 beats per minute. This compares more favorably to Yang and Graham's findings. This relationship merits 
further exploration. Additionally, Yang's sample was recruited from a YMCA, so these children were interested in physical activity and they chose to participate. This is a different situation from a physical education class, which would typically have a much more diverse cross section of students in terms of their interest in physical activity and fitness level (and thus, ability to sustain higher heart rates). The circumstances under which this study was undertaken differ markedly from a YMCA since teachers place expectations upon children to participate as a means to assess their progress in physical education. There is more incentive for school children to engage in the assigned tasks, and their time is much more structured. The use of a different heart rate monitor may be another reason for the differences in heart rates between the two studies. The Yang and Graham (2005) study used the chest strap model (Polar S810i). Chest strap models are often seen as the next best instrument to use after the electrocardiogram (Achten \& Jeukendrup, 2003), but they are much less practical in an elementary school setting. Students must place the strap next to the skin just below the nipple line, thus requiring time to get changed. With so little time dedicated to physical education (e.g. 30 minutes on average), it would have been difficult to permit a minimum of 20 minutes of active time for the participants had the chest strap model been chosen for this study. It was much more convenient to use the Cateye heart rate monitors, in which the device was simply secured around the waist with the sensor clipped to the earlobe (underneath or on top of clothing). More importantly, the Cateye heart rate monitor has been established in the literature as an equally valid instrument (Terbizan et al., 2002).

As indicated above, an interesting data trend to note is that of DDR at Trial 2, which showed a marked increase in mean heart rate over Trial 1. Subsequent trials 
demonstrated a gradual decrease in heart rate with a significant difference between Trial 2 and Trial 6. One explanation of these data involves the learning of DDR. The researcher postulates that students became more proficient in their movements after Trial 2 , and this resulted in a gradual decrease in the effort required to successfully respond to cues on the screen. Though students continued to challenge themselves by increasing the difficulty level of songs (e.g. from "beginner" to "light"), they had learned what movement strategies worked best to accumulate a high score. For example, a beginner player often comes back to the middle of the dance pad before responding to the next cue. More experienced players learn that this is not necessary; they can simply move from one arrow to another without the wasted movement of going back to the center of the pad.

An important observation that should be mentioned at this point is the fact that even though mean heart rates for DDR are significantly higher than volleyball and approached significance when compared to Tae-Bo, DDR remains an intermittent activity. Each song is approximately 90 seconds in length, after which follows a brief stoppage in play for participants to receive scores and make the next song selection. So, this activity would have an exercise-to-rest ratio of approximately $3: 1$ in most cases. If participants performed DDR for sustained periods of time with little or no rest, heart rates may be much higher than those reported in this study. However, the NASPE (2004) recommendations suggest intermittent activities so that children are not exerting themselves for extended periods of time. In future investigations, it would be anticipated that heart rate monitoring would be continuous throughout the exercise period, rather than simply an average heart rate for the whole period. This would provide more detailed information regarding heart rate fluctuations during physical education classes. Based on 
the heart rate results of this study, however, DDR is a suitable activity for fifth- and sixthgrade children.

It is not surprising that volleyball elicited the lowest heart rates of the three activities. However, heart rates were elevated to an extent that may have elicited healthrelated benefits (Kelly, 2000). Volleyball, by its very nature, is an intermittent, low intensity activity. This is particularly true for young students with lower skill levels. Volleyball rallies are usually very brief, with only one or two passes across the net before play is stopped due to a mishandled ball. Many students stand still while waiting for an opportunity to play the ball if it comes in their direction. The trend for heart rate over the six trials did not change significantly. This supports the observation that most sessions were similar in the way the lesson plan was implemented and the types of activities provided for students. The one exception was Trial 4 in which mean heart rate was markedly lower than that of the other trials. In that particular lesson, only one volleyball net was set up (e.g. full court), whereas in all other lessons the teachers used two nets. This dramatically affected the intensity of movement of the class as a whole. This result supports the pedagogical goal of increasing active time by decreasing wait time and increasing opportunities to manipulate the volleyball. One large game of volleyball did nothing to enhance health related fitness in this sample of fifth- and sixth-grade students.

To summarize the effect of activity type on heart rate in this sample of schoolaged children, DDR does not show similar heart rates to regular physical education and aerobic dance classes. It shows significantly higher heart rate means in fifth- and sixthgrade participants. This certainly has implications for teachers in terms of engaging students for sustained periods of time in physical education class. DDR is an appropriate 
activity for physical education classes and indeed, at home. It is an excellent way in which children can meet daily recommended levels of physical activity and to enhance cardiovascular fitness.

\section{Step Count}

DDR elicited the highest mean step counts, and these were significantly greater than both Tae-Bo and volleyball. Since DDR is an activity that is self-paced and selfmonitored (e.g. no direct teacher instruction), this result is particularly important for a number of reasons. DDR can increase physical activity levels without teachers having to insist upon student participation. It provides the participant with many choices in terms of level of difficulty, song selection, and fellow participants. It also allows teachers to remove themselves from direct instruction, and in doing so, frees them to assess student progress, interact with individual students, and perhaps even participate themselves!

One relevant finding with regard to step count is that there were no significant differences in step count between genders for any of the activity types. The literature suggested that boys accumulate more steps per day than do girls (Cardon \& De Bourdeauhuij, 2004; Pangrazi et al., 2003; Vincent et al., 2003). In the Pangrazi et al. (2003) study, mean step counts were reported for the entire day and not the intervention session alone (e.g. 15 minute activity session). It is therefore difficult to determine whether gender differences existed during the intervention itself. These researchers did report significant gender differences for daily step counts. McKenzie et al. (1995) noted that gender differences were less apparent in structured physical activity contexts than in unstructured free-play environments. The results of the current study agree with the findings of McKenzie and his colleagues; gender was not a factor during any of the 
physical education classes with respect to step count. Boys and girls accumulated similar step counts during each activity type. It would be interesting to monitor this same group of participants continually over a series of days to determine whether or not gender differences exist with respect to step count, particularly during DDR.

The step count trend over the six trials was significantly different for activity type. In looking at Figure 7, the trend for DDR step count rises over the intervention, with Trial 6 step count significantly higher than Trial 1 . This is an interesting finding given the heart rate trend is decreasing over trials. It is proposed that as participants become more competent in DDR, they are able to perform more steps with less effort. Stewart et al. (2004) reported a mean step count of 1,022 for fifth-grade students taking part in a 10 minute bout of moderate-vigorous physical activity during the school day. This equates to 102.2 steps per minute. The steps accumulated during physical education classes for all three activity types in this study were less than those reported in the Stewart study; volleyball (42.48 steps/minute), Tae-Bo (58.64 steps/minute), and DDR (61.07 steps/minute). The length of the lesson would definitely have affected this result. Participants, knowing that the exercise bout was only 10 minutes in length may exercise more intensely than those participants involved for a longer period of time, would accumulate more steps. Scruggs et al. (2005) reported a mean of 64.11 steps per minute in third grade participants. Although it is problematic to compare across grade levels because of developmental differences and lesson contexts, it does appear that both TaeBo and DDR compare favorably to the recommended step counts (e.g. 58-61 steps per minute) to achieve at least 10 minutes of MVPA in physical education class (Scruggs et 
al., 2005). This is particularly relevant given the high percentage of active time reported in the current study for all three activity types.

In examining the step counts for volleyball, there is a significant drop in step count at Trial 4, corresponding to the heart rate results. Again, at Trial 4, one volleyball net was used and this tremendously impacted step counts. This result indicated that students spent a lot of time standing around waiting to respond, an undesirable event in a physical education class.

The pedometers simply measure step counts; they do not measure the intensity of each step. If the goal of the physical education program is to simply get students more active, then the accumulation of steps is important. If, however, cardiovascular fitness is a goal the number of steps accumulated over time becomes important. Given that DDR elicited the highest step counts per minute and the highest heart rates, one could conclude that DDR would meet both goals. The results of this study support those of Yang and Graham (2005) in which DDR can be classed as a moderate-vigorous physical activity. Future research would attempt to address the use of pedometers during a controlled period of time. This would provide more information regarding the frequency of steps, and researchers may be able to make more conclusive statements regarding the intensity of exercise.

The pedometer has been validated in the literature as an effective tool to measure physical activity levels in children (Tudor-Locke et al., 2002). It is a particularly practical instrument for physical education classes. One issue of concern that should be addressed in future research is the ability of the pedometer to detect lateral movements. In the present study all three activity types involved movements in all directions, but the degree 
to which the pedometer was able to detect these movements is unknown. It is recognized that the pedometer likely missed many of the lateral movements, but because all three activity types did involve lateral movements it is suggested that the results were not significantly impacted. Considering the possibility that the results for step count may be an underestimation of actual events, the results become even more meaningful when presenting DDR as an activity eliciting high levels of MVPA.

The correlation coefficients identified significant relationships between step count and active time for all three activity types. This is not surprising given that both measures were obtained from the pedometers. In fact, the high correlations lend validity to the pedometer as an instrument to measure physical activity levels in children of this age group.

Participants engaging in DDR do not demonstrate similar step counts when compared to regular physical education and aerobic dance. Participants accumulated significantly more steps when engaged in DDR than when engaged in either Tae-Bo or volleyball. DDR can be an additional activity added to physical education programs to enhance physical activity levels of elementary students.

\section{Active Time}

A related and important factor for teachers to consider is the active time during physical education classes. Current research reports that students spent too much time listening to instructions or waiting to respond (McKenzie et al., 2000). The findings of the present study indicate that both DDR and Tae-Bo can tremendously increase active time in physical education class. When expressed as a percentage of total elapsed time, 
both Tae-Bo (70\%) and DDR (68\%) exceed the 35\% active time reported by Levin et al. (2001). Even volleyball active time (54\%) far exceeded that reported by these researchers. It should be noted that "active time", as measured by the pedometers, related to the time spent moving and accumulating steps. Pedometers cannot determine the intensity of exercise unless the number of steps accumulated is measured over time. When this is taken into consideration, the results of this study still compare favorably to the results reported by McKenzie et al. (2000) in which middle school students spent approximately $14.6 \%$ of physical education classes engaged in moderate-vigorous physical activity, and a further $33.8 \%$ of the time spent walking. The total active time of 48.4\% (McKenzie et al., 2000) remained lower than the results of the current study across all activity types. McKenzie et al. (2000) measured active time in a number of lesson contexts, and these authors emphasized the importance of this when examining the activity level of students. Lesson context is likely one of the key factors affecting the results in the present study. An activity like Tae-Bo does not have any breaks; it is a continuous activity that requires little management or teacher instruction, nor is there much opportunity for off task behavior. That would explain the high percentage of active time noted above. Additionally, students are much more likely to be actively engaged in preferred activities. Based on the observations of the researcher, participants preferred DDR and volleyball much more than Tae-Bo. It is only natural that students would be more active in physical education lessons that appeal to them for a variety of reasons; previous experience, skill level, fitness, motivation, attitude, self-efficacy and enjoyment to name a few. Welk (1999) stated that children usually pursue activities in which they believe they can be successful. Welk's predisposing factors, which include self-efficacy 
and enjoyment, are influential determinants in physical activity engagement and subsequent perseverance to continue activity. Both enjoyment and self-efficacy will be discussed in the sections that follow.

Percent active time for DDR was significantly greater than that for regular physical education (volleyball) in this study. Therefore, DDR would be an effective way to enhance levels of physical activity within physical education classes.

\section{Perceived Exertion}

Self-reported perceived exertion demonstrated significant changes over trials for activity type. As Figure 11 showed, rates of perceived exertion (RPE) for DDR remained between those for Tae-Bo and volleyball. According to the participants, Tae-Bo was the most fatiguing activity across all trials. The trend over trials for Tae-Bo indicates a gradual increase in RPE over the first four trials. This parallels the Tae-Bo heart rates over trials, which also showed a gradual increase. There was a slight drop in Tae-Bo RPE at Trial 5, but it still remained above the other two activity types. Based on the heart rate results, it would appear that the psychological processes (e.g. pain tolerance, selfefficacy, and mood) play a more dominant role in determining rates of perceived exertion in this sample than do physiological processes. As noted by Robertson (2004), both processes contribute to decisions about performance effort, but there remains a lot of inter-individual variability in how perceptual and physiological cues are interpreted.

DDR rates of perceived exertion remained relatively stable over the six trials, with only minor fluctuations. What is most interesting about these results is the relationship between RPE and heart rate for DDR. As mentioned in a previous section DDR elicited the highest heart rates. This was not true for RPE; DDR was significantly 
lower than Tae-Bo. By way of explanation, those participating in DDR did not realize how tired they had become. Participants became so engaged in the game that perceptual and physiological cues arising from exertion were masked. DDR distracted the participants from their true feelings of fatigue. This was not the case for Tae-Bo. Rates of perceived exertion were highest for Tae-Bo, even though music and video cues were involved. The type of music and video cues, however, differed significantly between the two activity types. It is obvious which activity was preferred by participants.

As reported by Robertson et al. (2005), perceived exertion showed a significant relationship with heart rate. The general trends for Tae-Bo and volleyball appear to agree with Robertson et al. (2005), in that heart rate measures paralleled RPE over trials as shown in Figures 13 and 14. It is clear, however, that the physiological cues have been masked to a certain extent by positive psychological cues when participants determined their levels of perceived exertion for DDR. The Pearson product-moment correlation coefficients did not support the significant relationship noted by Robertson and his colleagues (2005). Further examination of the psychological variables involved in DDR participation is warranted.

\section{Enjoyment}

The results for enjoyment revealed that DDR was the most popular activity, scoring approximately $34( \pm 2.1)$ out of a possible 35 points on the enjoyment scale. TaeBo was definitely unpopular with a mean score of $21( \pm 8.3)$ over the six trials. Volleyball was close to DDR with a mean score of $31( \pm 5.7)$. The standard deviations indicate that there was much more agreement in enjoyment scores for DDR than for the other two. Tae-Bo showed the greatest spread in scores. This agrees with the observations 
of the researcher, as some students (only a few) stated that they continued to enjoy TaeBo by Trials 5 and 6 . Similar to most offerings in the physical education curriculum, the students are mixed in their preferences for certain activities. Previous experience, perceived skill level, social dynamics, age, instructional style, and implementation strategies are just a few of the factors contributing to judgments about how enjoyable physical education can be for students. Welk (1999) has established enjoyment as a key variable in one's decision to engage in physical activity. Certainly the fact that DDR was a novel activity played a role, but Tae-Bo was also new to this group of students.

It is interesting to note that Tae-Bo elicited the lowest enjoyment scores and the highest rates of perceived exertion. The correlation coefficient for these two variables approached a significant negative relationship, indicating a decreasing trend for enjoyment with an increasing trend for perceived exertion. Because participants became bored with the activity, their attention might have turned toward other things, namely, their psychological and physiological feelings of fatigue. Alternatively, their psychological feelings of well-being while engaged in Tae-Bo were not sufficient to mask the physiological and psychological cues arising from exertion. Finally, the participants might have found the movements too difficult to perform, thus increasing perceived exertion (and frustration) and reducing their enjoyment of the activity. Welk (1999) proposed that children will pursue activities in which they can be successful. The two questions he posed are critical factors in deciding upon physical activity behavior; “Am I able?", and "Is it worth it?" Based on the Tae-Bo results, many of the participants might have answered "no" to both questions. Another issue related to these two questions is this; the feedback participants received during Tae-Bo was significantly different from 
that of the other two activities. In DDR, feedback was immediate through grade score display at the conclusion of each song, and in volleyball, feedback was related to successful game play. In Tae-Bo, however, feedback was limited to the encouraging remarks of the teacher. The participants simply tried their best to follow the leader on the screen. Students were not able to make judgments about their success and therefore the value of the activity was in question. This definitely would have impacted enjoyment scores and subsequent participation in Tae-Bo. The relationship between enjoyment and perceived exertion is of interest to physical educators planning appropriate physical activities for their students.

Dishman et al. (in press) reported a mean enjoyment score of $29.7 \pm 5.5$ for a group of sixth-grade females during physical education class. The enjoyment questionnaire for the Dishman study was administered during regular physical education class, and was the same one as that used in the present study. The content of the physical education classes was not indicated, so it is difficult to compare the two samples. It does suggest, however, that the two groups differed in their enjoyment of physical education. The possible reasons why the present group enjoyed DDR and volleyball are discussed below.

Both volleyball and DDR displayed very high scores for enjoyment yet it was DDR that had the greatest effect on heart rates. Students were enjoying themselves despite the fact that they were exercising intensely while engaged in DDR. In most sports and activities it would be anticipated that enjoyment would decrease as exercise intensity increased (and thus feelings of fatigue and discomfort), but this was not the case for DDR. As noted in the previous section addressing perceived exertion, feelings of 
discomfort appear to have been masked because participants have become so engaged in the game.

Welk (1999) highlighted enjoyment as a major predisposing factor in predicting physical activity in children. The results of this investigation suggest that DDR would be an appealing way to engage youth in physical activity. While it is difficult to generalize to other populations, current results and anecdotal evidence leads the researcher to propose that DDR would appeal to children of all ages. Postulating the reasons why participants enjoy DDR so much would be a relevant issue for future research, and for developing other engaging activity choices in physical education classes. DDR is a relatively "new" technology, and anything that combines "new" and "technology" is of great interest to this generation of technologically oriented children. Children are video game literate, as $92 \%$ of children aged 2-17 play video games (National Institute on Media and the Family, 2001). Based on the researcher's involvement in and experience with DDR, the following are proposed factors that have placed DDR at the top of the enjoyment list for fifth- and sixth-grade students: (a) DDR involves technology, (b) DDR involves music and rhythm, (c) DDR involves choice, (d) DDR is a social activity, (e) DDR is self-paced, (f) DDR requires little teacher intervention, (g) DDR measures success immediately, and (h) DDR requires minimal skill to be successful. It would be important to investigate these factors to better inform teachers of ways in which they can improve their planning, implementation, and evaluation of physical education so that their learners will better appreciate the value of a physically active lifestyle. Funk (1993) suggested that video game play habits may reflect future behaviors in some children or adolescents. Children who develop an appreciation for, and an interest in, physically 
interactive video games may develop future behaviors that enhance their personal health and fitness.

Macfarlane and Kwong (2003) found lowered enjoyment levels in physical education classes that lacked variety and were highly structured. DDR is certainly an activity that contrasts starkly with a highly structured, monotonous physical education class. DDR is largely a student-directed activity, and the game options are numerous (e.g. song selection, difficulty level, mode of play). Prochaska et al. (2003) noted a consistent decline in physical education enjoyment from fourth- through sixth-grade. The implementation of a varied program that included DDR would be a positive step towards preventing or slowing this decline.

Another important question to answer in subsequent investigations based on the results of the present study is to determine the degree to which enjoyment scores might be maintained over time. This question is a critical one given the importance placed upon developing physically active individuals for lifelong health. The correlation coefficient between enjoyment score and elapsed time revealed a significant negative relationship, $(r=-.345)$, indicating a possible trend for decreased enjoyment as the elapsed time for the exercise session increased. Perhaps future investigations would seek to determine an optimal exercise time for DDR without compromising its enjoyment value. In doing so, participants would continue to look forward to subsequent DDR sessions. It is important to note that the game developers continue to update the game $\mathrm{CD}$ with new music, different ways to play the game, and enhanced graphics. The technology of the game platforms, Xbox ${ }^{\circledR}$ and PlayStation ${ }^{\circledR 2}$, continue to evolve as well with updated capacity, online availability and memory capability. DDR is just one of many "exergames". Other 
similar versions exist, some with bicycles linked to the video screen where participants must pedal vigorously to continue the game. This new wave of interactive technology is only just beginning. There are myriad possibilities, so the risk of boredom with this type of activity is only limited by the available resources.

Tae-Bo had significantly lower enjoyment scores than both DDR and volleyball. In examining the trend over the six trials in Figure 15, there was a drop in enjoyment from Trial 1 to Trial 6 indicating participants became less and less thrilled with Tae-Bo. This is not surprising given that the same video tape was used for each session. Even though different sections of the tape were used each lesson student quickly got bored with following the leader on the screen. Further, the correlation coefficient between enjoyment and elapsed time was not significant for Tae-Bo, indicating the possible effect of the shorter exercise session.

In comparing Tae-Bo to the list above, there are several key factors missing in the Tae-Bo sessions. Firstly, Tae-Bo is not self-paced; children must make every attempt to keep up to the leader. Secondly, participants do not get immediate feedback other than that provided by the teacher. This feedback was in the form of encouragement to keep going as long as possible. And thirdly, participants do not have any choice in terms of difficulty level, pace, or music selection. It may be that the music selections on the TaeBo tape were not appealing to this age group. These findings are significant for teachers. Though Tae-Bo can provide some variety in program offerings, it may be an activity that should be used judiciously when attempting to keep enjoyment levels high in physical education classes. 
Volleyball is a sport that is found in most physical education programs. It is an activity that obviously requires a certain degree of skill before competent performance results. Elementary school physical education classes involving a game of volleyball usually result in the ball crossing the net only one or two times. As an example, one team would serve; the receiving team may be able to contact the ball once or twice to put it back over the net before the ball is mishandled and play stops. Unless teachers of volleyball use a variety of drills and volleyball-like activities to keep activity high, a volleyball game is an inadequate way to actively engage students. Nonetheless, volleyball remains a popular activity. The researcher suspects that because volleyball requires little energy output, many students enjoy the game. Even students who lack the necessary skills enjoy volleyball. They can still feel a part of the action when standing on the court, knowing that a more skilled player could step in front of them to play the ball. It has been the experience of the researcher that in a traditional six-on-six volleyball game, there may be players who never touch the ball. In this particular study, the teachers involved recognized this possibility, and adjusted their lesson plans accordingly. They set up two volleyball nets and reduced the number of players on the court (except for Trial 4). This increased contact time and activity level. Enjoyment scores were not affected by the use of a single net at Trial 4, but it certainly impacted heart rates. This suggests that regardless of what volleyball-related activities are provided for students, those who like the game will enjoy volleyball classes. It should be noted that many of the participants (e.g. 10) in the current study were also members of the school volleyball team, so they already had a predisposition toward volleyball enjoyment. This likely had an impact on the enjoyment scores, and indeed, many of the other dependent variables. 
In summary, participants in this study enjoyed DDR more than an activity which they already enjoyed - volleyball. Tae-Bo was an activity that was not popular in this group of fifth- and sixth-grade students, particularly as time passed. Enjoyment is a significant correlate with intention to engage in moderate-to-vigorous physical activity, as it provides immediate reinforcement for being physically active (Bungum et al., 2000; Motl et al., 2001). This is an important factor to consider for those providing physical activities for children. Based on the results of this study, the hypothesis that students engaging in DDR will experience higher levels of enjoyment than both regular physical education and Tae-Bo has been accepted.

\section{Self-Efficacy}

Self-efficacy did not change significantly over the course of the six week intervention. Based on the highest possible score of 40, participants perceived themselves to have a high level of self-efficacy with a mean score of 31 . This compares well with Dishman et al. (in press), who used the same instrument with a group of sixth-grade females and reported a mean score of $30.2 \pm 6.2$. In other words, the participants in this study strongly believed in their ability to successfully overcome barriers in order to engage in physical activity. According to Dishman et al. (in press), enhancing selfefficacy for overcoming barriers might represent an important initial target for physical activity interventions in early adolescence. An examination of each of the types of barriers noted in the questionnaire revealed some specific areas in which barriers may be addressed in future. The most significant barrier that may be modifiable is television/video game use. Eighteen percent of participants reported they would not give up television or video game use in place of physical activity. Screen time continues to 
usurp time that may be used for physical activity (Vandewater et al., 2004). An engaging video game like DDR may combine the best of both worlds for these youth. There were two other barriers identified that precluded physical activity: homework and busy lifestyles. While it is certainly not the intent of this paper to decry the importance of homework or leading active lives, these two issues may suggest addressing self management strategies to assist in making time for physical activity. Dishman et al. (in press) found that self management strategies like goal-setting, thought awareness, and planning significantly mediated barriers self-efficacy. Accordingly, these authors suggested that the use of self-management strategies might enhance self-efficacy. It would be a worthwhile exercise to further investigate this relationship, as it may be particularly relevant for teachers and students in the educational setting. As a footnote, it is interesting to note that adults often use the same excuses for not exercising; "I have too much work to do", and "I am too busy doing other things".

Self-efficacy was measured prior to and immediately following the intervention at the same time of day. It was not attached to a specific physical education class. Thompson et al. (1998) found self-efficacy to be the most significant predictor of intention to be active when self-efficacy scores were measured immediately before the activity. The Thompson study, however, did not change physical education lessons. Because the participants in this study were involved in an intervention that included a dramatic modification of physical education classes, it was felt that the impact of the intervention would be most evident if self-efficacy was measured at a time unrelated to physical education class. There may be a numerous reasons why self-efficacy did not change significantly. One possible reason is that the teachers involved have been positive 
role models in encouraging physical activity in the school, not just in physical education class but also in extracurricular activities. The fact that so many participants were also members of the school volleyball team is one example of student interest in sport and physical activity. Many other athletic events were planned for the school year, so the importance of physically active lifestyles was embraced at this school. Other factors may have included the family; $73 \%$ of participants indicated they would have no trouble asking a parent or other adult to do physically active things. Eighty-five percent said they could ask a friend to be physically active during free time. Finally, most students $(82 \%)$ reported they possessed the coordination necessary to be physically active. Accordingly, participation rates were high in all activity types, and the corresponding enjoyment scores and heart rates indicated that participants were confident, active members of each physical education class.

Participants did not demonstrate higher levels of self-efficacy at the end of the study than at the beginning. A longer intervention would more thoroughly explore the relationship between DDR and self-efficacy. With DDR becoming more widely available for home use, arcade use, and school use, youth will begin to recognize that it can be an exciting way to get active. Undoubtedly, given the opportunity, the participants in the present study would eagerly engage in DDR in the future.

\section{Limitations}

There are a number of limitations to this research which must be addressed. The teachers selected for the study were highly motivated and dedicated to the project, but one teacher was much more diligent in carrying out the procedures than the other teacher. 
For example, one teacher maintained a strict routine for the distribution of the instruments, the completion and collection of questionnaires, and pushing of the correct buttons on the instruments. She made sure that each and every student activated the heart rate monitors and pedometers as they entered the gymnasium. She strictly enforced the completion of the perceived exertion and enjoyment questionnaire immediately following each physical education class. The other teacher did make strong efforts in these areas, but she was not as consistent. This might have been a contributing factor in the omission of so many data sets in this class. One teacher was absent on two occasions, and this certainly affected the behavior of the students in that class. A supply teacher does not have the same degree of classroom control as the regular teacher, so students may not have been as compliant on those days. This created more data collection errors. Every effort was made by the researcher (who was present on each day of data collection) to support the teachers and assist them with the data collection.

The participants themselves were very agreeable to wearing the heart rate monitors and the pedometers, but several issues arose as data collection progressed, and these impacted data collection. Though every effort was made to instruct participants in correct placement and use of the pedometers and heart rate monitors, some pedometers were not re-set correctly or at the right time, nor were heart rate monitors set to record mean heart rates. The teachers and the researcher, after one or two sessions, made sure to double check the instruments while the class was proceeding to ensure that data were being recorded properly. The researcher would be scanning the class frequently to replace a dangling pedometer, or ensure that a heart rate monitor was recording properly. Despite the best intentions of all concerned, some data were lost or negatively affected. 
In terms of participant reactivity to the instruments, this was reduced to some extent by introducing and using the pedometers and heart rate monitors during the familiarization sessions. By the time the study began, students were competent in the use of the instruments, and they simply regarded them as essential pieces of equipment for physical education class. Many students were curious about their results, so on occasion they would check their pedometers and/or heart rate monitors during class. This obviously distracted them from the assigned task. More frequently, students would want to know their step counts at the end of class, so before they placed the pedometers on the stage, they would open them to check. This resulted in some participant awareness of scores, which might have subsequently impacted participation in their next physical education class or their responses on the questionnaire. Usually they simply wanted to compare their step counts with those of their peers. In future, a sealed pedometer or an ankle-worn pedometer may reduce the tendency for participants to check their scores. The heart rate monitors were of less interest to participants. Having sealed heart rate monitors would have reduced the reactivity and prevented any data from being disclosed to participants. For the most part, however, students quickly became accustomed to the instruments and participated fully in their classes. They demonstrated good compliance in following instructions for using the instruments.

The schedule of activities for physical education class was not posted, so participants did not know what to expect each day. By the time each physical education class began, they obviously knew (due to equipment set-up). Because the sixth-grade class followed the fifth-grade class, the sixth-grade participants knew further ahead of time what was on the agenda for physical education class. Advanced knowledge of the 
activity might have adversely affected results, particularly with respect to enjoyment. Knowing that the activity was not DDR, some participants might have been disappointed and therefore not put forth as much effort in the class. One strategy to have addressed this might have been to prepare a separate rotation of activities for each grade level. As a result of timing and logistical issues during implementation, this was not done. An alternative study design that focused only on DDR might have reduced the effect of prior knowledge.

One major source of data collection error was the absenteeism that occurred in the sixth-grade class. Of the original 26 participants the data from 12 were used in this study. Missing data points did occur frequently, and a participant's data set was not accepted if more than $11 \%$ of data were missing. This meant that of the 108 data points collected for each participant, only 12 missing points would be acceptable. Anything over 12 resulted in the deletion of that participant's data set. Missing data points were replaced with averages of the remaining data points for that particular variable. For example, if one participant missed heart rate for one session of DDR, the remaining five heart rates would be averaged together, and that value entered as the sixth data point. This reduced the power of the repeated measures design, as trends would be flattened. One way to have addressed this would have been to average the two adjacent values and enter that number as the missing data point. The accuracy of the recording of data may have been a source of error. The researcher had one assistant in the recording of step counts and active time from the pedometers. Errors in the written transfer of data from the pedometers and heart rate monitors to the recording sheets, and subsequent entry into the electronic database were thus minimized. All data were verified before data analysis took place. 
There were some threats to internal validity as a result of instrumentation issues. The pedometers, if placed properly at the beginning of each class, were generally very reliable instruments over the course of the intervention. Most errors occurred because of poor pedometer placement. In some cases the midsection of the participant caused the pedometer to roll horizontally, thus rendering it ineffective in step counting. Measures below 100 steps were not accepted. The heart rate monitors, however, were not as reliable. The sensitivity of the earlobe clip was such that in some participants, the monitor could not get an accurate heart rate. Some of the heart rates recorded were below that which would be considered physiologically possible. It is suggested that in some cases, data collection was momentarily interrupted due to the inability of the earlobe sensor to detect the heart rate. This would have decreased the overall heart rate average at the end of the exercise session. Heart rates below 80 beats per minute were not accepted, as this was above the mean resting heart rate for this sample. One other issue that came to light after several trials was the default alarm setting on the heart rate monitors. Coming from the factory, these devices are set so that if heart rates reach 180 beats per minute, an alarm sounds to warn the wearer that he or she has gone beyond a target heart rate zone. The sound of the alarm going off during class was somewhat distracting initially, but participants soon became accustomed to this sound, and they were shown how to deactivate the alarm. The use of sealed accelerometers, in addition to, or in place of pedometers and/or heart rate monitors in future may provide more accurate information regarding the intensity of exercise. One other key limitation that should be addressed in future research is that of careful control of the total elapsed time for each trial. Because there were variations in the length of each trial among and between activity types, the 
step count results may not necessarily reflect the true differences that existed. Controlling for elapsed time or counting steps per unit time would be more appropriate.

An important factor to consider in future relates to controlling the total elapsed time for each lesson. As indicated in the previous chapter, there were significant differences in lesson length between activity types. The major reason for Tae-Bo sessions being shorter than the other two was related to enjoyment. Participants simply did not enjoy Tae-Bo, and it was a challenge for the teacher to motivate students to keep going for any longer than about 22 minutes. This duration, however, did exceed the minimum of 20 minutes required for each lesson. The lower total lesson time might have actually inflated the significance of the Tae-Bo results across many of the dependent variables. For example, heart rates might have been much lower had participants been asked to participate for several more minutes. In fact, enjoyment scores might have been even lower had participants been asked to continue participating for the additional time. This is something that would need to be addressed in future research.

The questionnaires and the timing of their completion is another threat to internal validity, particularly for one class. In all cases, the perceived exertion and enjoyment scale questionnaire was completed in the classroom after students had left the gymnasium. This would have given participants a brief period of time to perhaps "cool down" or forget their intensity of effort. It might also have given participants time to chat with one another about their experience, thus impacting scores. It would have been ideal to implement the questionnaires immediately before participants left the gym, but this was not practical from an organizational point of view. In some situations participants immediately went out for their 15 minute recess and didn't complete the questionnaire 
until they came back inside. Because the questionnaires were used following each of the 18 trials, fatigue or prior history with the questionnaire might have impacted subsequent responses. Though the teachers involved continued to emphasize the importance of answering the questionnaires honestly and thoroughly for the duration of the study, participants might have become tired of the process and therefore not provided accurate responses.

A larger sample size for the between-group comparisons would have strengthened the results of the study. For example, when blocked by grade level, only 12 participants were in the sixth-grade. Had the researcher been able to retain the 14 discarded sets of data in this group, the trends between groups might have resulted in significant findings.

Finally, the generalizability of these results is difficult for several reasons. This convenience sample was not culturally diverse, so it would be inappropriate to generalize these findings to a more heterogeneous population. The fact that this sample was one of convenience also limits the findings to this particular population. The levels of physical activity, number of hours of weekly physical education, and the physical activity opportunities available may not necessarily be consistent across the province of Ontario, across Canada, or the United States. As indicated in previous sections, this group of participants reported high levels of physical activity outside of school hours but they exhibited elevated body mass indices. According to the research literature, this may be an atypical population, and therefore generalizability is not possible. Future random sampling of more diverse populations from different regions would be an appropriate goal. 


\section{Future Directions}

The study design permitted within-group comparisons as well as between-group comparisons. This was an optimal design for the convenience sample selected. Future research would expand upon this design to include more grade levels, a more diverse population, and a variety of geographic locations. Other study designs might compare many of the same dependent variables in schools with DDR fully integrated into the physical education versus schools without DDR in their curriculum. This may be one way to further explore the relationship between DDR and physical activity intensity, enjoyment and self-efficacy. With a greater range of grade levels involved, a clearer picture may emerge regarding when DDR might be most appropriate in engaging children in physical activity. There may be optimal stages in childhood development when enjoyable activities may have a lasting effect on intention to engage in subsequent physical activity. This would be a relevant finding for educators, parents, and fitness promoters.

Because DDR is a student-directed activity, research opportunities exist to compare DDR (and other physically interactive video games) to more traditional curricular offerings. This might include comparisons to team-oriented sports, or to teaching methods involving direct instruction. The degree of student choice in physical education classes would be an interesting line of research. DDR is highly student-directed in terms of opportunities to respond, self-selection of intensity level and song choice. This could be compared to activities in physical education that are very structured and directed by teachers. Dependent variables may be related to the intensity of physical activity as well as the psychological factors discussed in the present study. There are also 
opportunities to examine other dependent variables related to motivation, skill acquisition, and rhythm. Further, it would be important to determine the relationship between regular participation in DDR and learning. For example, researchers might explore whether or not DDR has an impact on attentional focus or achievement in academic subjects.

In the present study, volleyball happened to be the regular curriculum offering at that time of the year. In subsequent studies, DDR would be compared to more physically active units of study, such as fitness, basketball, team handball, etc. Additionally, other methods of assessing levels of physical activity would be considered. Sealed instruments, including accelerometers or ankle-mounted pedometers may provide more reliable information. More thorough assessment of self-reported physical activity levels could be validated against directly measured daily physical activity. A longitudinal study to investigate to the lasting effect of DDR enjoyment and physical activity levels would also be appropriate to determine whether this innovative technology to physically engage children has endurance over a period of months or years.

An issue of great importance to educators is the rising cost of athletic equipment. For most schools, the provision of one dance pad per student would be cost prohibitive. Because of this, the practicality of implementing DDR in this way comes into question. The storage and security of equipment is also a concern in most elementary schools. There are some possible solutions to these problems that have emerged as a result of this research. If schools had only one or two game units with 4-8 pads, DDR could be used as one station of a multi-station circuit in physical education class. A single DDR unit and pad (with headphones) could be placed at the back of a classroom for students to 
integrate physical activity into classroom activities. Finally, DDR can be projected onto the gymnasium wall so all students can participate in DDR using the same song, yet only those students on the active pads would receive scores. A dedicated, supervised space for DDR would permit access during curricular, extracurricular, and recess periods of the entire school day. Future exploration of these possibilities would be relevant for teachers interested in integrating student-centered technology in their physical education curriculum. On a positive note, the Near North District School Board is investigating the possibility of purchasing some DDR units to be shared amongst Board schools. Because of the newly legislated mandatory 20 minutes of daily physical activity for all elementary school children (beyond regular physical education classes) in Ontario, there may be future research opportunities in this area.

Teachers continue to seek innovative ways to engage their students in physically active pursuits with an ultimate goal toward impacting adult lifestyle choices. Video game technology continues to evolve to meet the ever-changing interests of children. Dance Dance Revolution represents an entirely different yet innovative activity to engage children that combines the allure of video game technology with large muscle movements. DDR is associated with heightened levels of physical activity sufficient to accrue health-related benefits in children. Perhaps the most exciting finding in this study was that students exercised at moderate-to-vigorous levels without recognizing the effort required to play DDR. This finding has far-reaching implications for teachers who struggle to maintain continuous activity levels in their classes. DDR may unwittingly contribute to the reduction of the number of overweight and obese children in schools because it was found to be such an enjoyable activity for all students. DDR is inclusive: it 
requires only basic motor skills, it engenders quick success, and it provides immediate feedback. These are key ingredients for activating youth in physical education, and indeed, beyond the schools. More research in this area will contribute to greater understanding of the complex issues surrounding physical activity engagement. One pivotal issue now is accessibility. In providing opportunities for children of all ages to participate in DDR, schools could play a more significant leadership role in reducing obesity and encouraging active lifestyles. 


\section{References}

Achten, J., \& Jeukendrup, A.E. (2003). Heart rate monitoring: Applications and limitations. Sports Medicine, 33, 517-538.

Allison, K.R., Dwyer, J.J.M., \& Makin, S. (1999). Self-efficacy and participation in vigorous physical activity by high school students. Health Education and Behavior, 26(1), 12-24.

Anand, S., \& Krosnick, J.A. (2005). Demographic predictors of media use among infants, toddlers, and preschoolers. American Behavioral Scientist, 48, 539-562.

Anderson, C.A. (2004). An update on the effects of playing violent video games. Journal of Adolescence, 27(1), 113-122.

Anderson, W.G. \& Barrette, G. (1978). What's going on in the gym: Descriptive studies of physical education classes. Motor Skills into Practice Monograph, 1-81.

Armstrong, N., Welsman, J.R., \& Kirby, B.J. (2000). Longitudinal changes in 11-13year-olds’ physical activity. Acta Paediatrica, 89: 775-780.

Bailey, R.C., Olson, J., Pepper, S.L., Porszaz, J., Barstow, T.J., \& Cooper, D.M. (1995). The level and tempo of children's physical activities. Medicine and Science in Sports and Exercise, 27, 1033-1041.

Bandura, A. (1977). Self-efficacy: Toward a unifying theory of behavioral change. Psychological Review, 84, 191-215.

Bandura, A. (1989). Human agency in social cognitive theory. American Psychologist, 44, 1175-1184. 
Bandura, A. (1997). Self-efficacy: The exercise of control. New York: Freeman and Company.

Beets, M.W., Patton, M.M., \& Edwards, S. (2005). The accuracy of pedometer steps and time during walking in children. Medicine and Science in Sports and Exercise, 37, 513-520.

Berger, B.G. (2004). Subjective well-being in obese individuals: The multiple roles of exercise. Quest, 56, 50-76.

Best, R.W., \& Steinhardt, M.A. (1991). The accuracy of children's counting of exercise heart rates. Pediatric Exercise Science, 3, 229-237.

Brobst, Y.S. (2002). An alternative to interactive metronome for my child. Autism Network Resources for Physicians. Retrieved June 10, 2004, from http://home.san.rr.com/autismnet/ddr.html.

Bungum, T., Dowda, M., Weston, A., Trost, S.G., \& Pate, R.R. (2000). Correlates of physical activity in male and female youth. Pediatric Exercise Science, 12, 71-79.

Burton, C.J. (1996). The heart rates of elementary school children during physical education classes. [Abstract]. International Institute for Sport and Human Performance, UO96\#360.

Cardon, G. \& De Bourdeaudhuij, I. (2004). A pilot study comparing pedometer counts with reported physical activity in elementary schoolchildren. Pediatric Exercise Science, 16, 355-367.

Casperson, C.J., Powell, K.E., \& Christenson, G.M. (1985). Physical activity, exercise, and physical fitness: Definitions and distinctions for healthrelated research. Public Health Report, 100, 126-131. 
Centers for Disease Control and Prevention. (2003). Physical activity levels among children aged 9-13 years - United States, 2002. MMWR, 52, 785-788.

Centers for Disease Control and Prevention. (2001). Body mass index. Retrieved April 30, 2006, from http://www.cdc.gov/nccdphp/dnpa/bmi/childrens_BMI/about_childrens_BMI.htm

Chase, M. (1998). Sources of self-efficacy in physical education and sport. Journal of Teaching in Physical Education, 18, 76-89.

Corbin, C.B., Pangrazi, R.P. \& Welk, G.J. (1994). Toward an understanding of appropriate physical activity levels for youth. President's Council on Physical Fitness, 1, 1-8.

Council for Physical Education for Children. (1998). Physical activity for children: A statement of guidelines. Reston, VA: NASPE Publication.

Cowden R.D., \& Plowman, S.A. (1999). The self-regulation and perception of exercise intensity in children in a field setting. Pediatric Exercise Science, 11, 32-43.

Crocker, P.R.E., Bouffard, M., \& Gessaroli, M.E. (1995). Measuring enjoyment in youth sport settings: A confirmatory factor analysis of the physical activity enjoyment scale. Journal of Sport and Exercise Psychology, 17, 200-205.

Cuddihy, T.F., Pangrazi, R.P., \& Tomson, L.M. (2005). Pedometers: Answers to FAQs from teachers. Journal of Physical Education, Recreation and Dance, 76 (2), 36-40.

Dietz, W.H. (2004). The effects of physical activity on obesity. Quest, 56, 1-11. 
Dishman, R.K., Motl, R.W., Sallis, J.F., Dunn, A.L., Birnbaum, A.S., Welk, G.J., Beimo-Rung, A.L., Voorhees, C.C., \& Jobe, J.B.. (in press). Self-management strategies mediate self-efficacy and physical activity. American Journal of Preventive Medicine.

Dishman, R.K., Motl, R.W., Saunders, R., Felton, G., Ward, D., Dowda, M., \& Pate, R.R. (2005). Enjoyment mediates effects of a school-based physical-activity intervention. Medicine and Science in Sports and Exercise, 37, 478-487.

DuRant, R.H., Baranowski, T., Davis, H., Thompson, W.O., Puhl, J., \& Greaves, K.A. (1993). Reliability and variability of heart rate monitoring in children. Medicine and Science in Sports and Exercise, 25, 389-395.

Emes, C.E. (1997). Is Mr Pac Man eating our children? A review of the effect of video games on children. Canadian Journal of Psychiatry, 42, 409-414.

Erdmann, L.D., Dolgener, F.A., \& Hensley, L.D. (1998). A comparison of postexercise heart rates by telemetry and self-pulse palpation in middle school-age boys. Measurement in Physical Education and Exercise Science, 2, 199-204.

Faigenbaum, A.D., Milliken, L.A., Cloutier, G., \& Westcott, W.L. (2004). Perceived exertion during resistance exercise by children. Perceptual and Motor Skills, 98, $627-637$.

Faith, M.S., Berman, N., Heo, M., Pietrobelli, A., Gallagher, D., Epstein, L.H., Eiden, M.T., \& Allison, D.B. (2001). Effects of contingent television on physical activity and television viewing in obese children. Pediatrics, 107, 1043-1048. 
Feltz, D.L. \& Chase, M.A. (1998). The Measurement of self-efficacy and confidence in sport. In J.L.Duda (Ed.), Advances in sport and exercise psychology measurement. (pp. 65-80). Morgantown, WV: Fitness Information Technology.

Funk, J. B. (1993). Reevaluating the impact of video games. Clinical Pediatrics, 32, 8690.

Gilbert, J.A. (2005). Using target heart-rate zones in your class. Journal of Physical Education, Recreation and Dance, 76, 22-26.

Gortmaker, S.A., Must, A., Sobol, K., Peterson, G., Colditz, G., \& Dietz, W. (1990). Television viewing as a cause of increasing obesity among children in the United States. Archive of Pediatric Adolescent Medicine, 150, 356-362.

Groslambert, A., Hintzy, F., Hoffman, M.D., Dugue, B., \& Rouillon, J.D. (2001). Validation of a rating scale of perceived exertion in young children. International Journal of Sports Medicine, 22, 116-119.

Guerra, S., Santos, P., Ribeiro, J.C., Duarte, J.A., Mota, J., \& Sallis, J.F. (2003). Assessment of children's and adolescents' physical activity levels. European Physical Education Review, 9(1), 75-85.

Hampson, D.B., Gibson, A.S., Lambert, M.I., \& Noakes, T.D. (2001). The influence of sensory cues on the perception of exertion during exercise and central regulation of exercise performance. Sports Medicine, 31, 935-952.

Haninger, K., \& Thompson, K.M. (2004). Content and ratings of teen-rated video games. Journal of the American Medical Association, 291, 856-955. 
Hedley, A.A., Ogden, C.L., Johnson, C.L., Carroll, M.D., Curtin, L.R., \& Flegal, K.M. (2004). Prevalence of overweight and obesity among US children, adolescents, and adults, 1999-2002. Journal of the American Medical Association, 291, 2847-2850.

Hoos, M.B., Gerver, W.J.M., Kester, A.D. \& Westerterp, K.R. (2003). Physical activity levels in children and adolescents. International Journal of Obesity, 27, 605-609.

Houghton, S., Milner, N., West, J., Douglas, G., Lawrence, V., Whiting, K., Tannock, R., \& Durkin, K. (2004). Motor control and sequencing of boys with attention-deficit/hyperactivity disorder (ADHD) during computer game play. British Journal of Educational Technology, 35(1), 21-34.

Johns, D.P., Ha, A.S.C. \& Macfarlane, D.J. (2001). Raising activity levels: A multidimensional analysis of curriculum change. Sport, Education and Society, 6, 199-210.

Kautiainen, S., Koivusilta, L., Lintonen, T., Virtanen, S.M., \& Rimpela, A. (2005). Use of information and communication technology and prevalence of overweight and obesity among adolescents. International Journal of Obesity, 29, 925-933.

Kelly, L.E. (2000). Patterns of physical activity in 9-10-year-old American children as measured by heart rate monitoring. Pediatric Exercise Science, 12, 101-110.

Kendzierski, D. \& DeCarlo, K.J. (1991). Physical activity enjoyment scale: Two validation studies. Journal of Sport and Exercise Psychology, 13, 50-64. 
Kilanowski, C.K., Consalvi, A.R., \& Epstein, L.H. (1999). Validation of an electronic pedometer for measurement of physical activity in children. Pediatric Exercise Science, 11, 63-68.

Kimiecik, J. \& Harris, A.T. (1996). What is enjoyment? A conceptual/definitional analysis with implications for sport and exercise psychology. Journal of Sport \& Exercise Psychology, 18, 247-263.

Konami ships Dance DanceRevolution Ultramix3 to retail stores nationwide. (2005, November 15). Retrieved April 6, 2006, from http://www.konami.com/gs/newsarticle.php?id=662.

Leger, L., \& Thivierge, M. (1988). Heart rate monitors: Validity, stability, and functionality. The Physician and Sportsmedicine, 16, 143-151.

Levin, S., McKenzie, T.L., Hussey, J.R., Kelder, S.H., \& Lytle, L.A. (2001). Variability of physical activity during physical education lessons across elementary school grades. Measurement in Physical Education and Exercise Science, 5, 207-218.

Macfarlane, D., \& Kwong, W.T. (2003). Children's heart rates and enjoyment levels during PE classes in Hong Kong primary schools. Pediatric Exercise Science, 15, 179-190.

Mahon, A.D., Gay, J.A., \& Stolen, K.Q. (1998). Differentiated ratings of perceived exertion at ventilatory threshold in children and adults. European Journal of Applied Physiology, 18, 115-120. 
Martin, B., Grisson, T., Ward, P., \& Lenders, N.Y. (2003). Effects of wearing heart rate monitors on elementary students' activity levels during a team handball unit. [Abstract]. Research Quarterly for Exercise and Sport, 74, A-49.

McAuley, E., \& Mihalko, S.L. (1998). Measuring exercise-related self-efficacy. In J.L. Duda (Ed.), Advances in sport and exercise psychology measurement. (pp. 371390). Morgantown, WV: Fitness Information Technology.

McGraw, T., Burdette, K. Seale, V., \& Gregg, S. (2002). The effects of pervasive, consumer-based interactive multimedia games on the reading disorders of ADHD children. Insight, 2, 5-30.

McKenzie, T.L., Feldman, H. Woods, S.E., Romero, K.A., Dahlstrom, V., Stone, E.J., Strikmiller, P.K., Williston, J.M., \& Harsha, D.W. (1995). Student activity levels and lesson context during third-grade physical education. Research Quarterly for Exercise and Sport, 66, 184-193.

McKenzie, T.L., Marshall, S.J., Sallis, J.F., \& Conway, T.L. (2000). Student activity levels, lesson context, and teacher behavior during middle school physical education. Research Quarterly for Exercise and Sport, 71, 249-259.

McMurray, R.G., Harrel, J.S., Deng, S., Bradley, C.B., Cox, L.M., \& Bangdiwala, S.I. (2000). The influence of physical activity, socioeconomic status, and ethnicity on the weight status of adolescents. Obesity Research, 8, 130-139.

Morgan, C.F., Jr., Pangrazi, R.P., \& Beighle, A. (2003). Using pedometers to promote physical activity in physical education. Journal of Physical Education, Recreation and Dance, 74(7), 33-38. 
Motl, R.W., Dishman, R.K., Saunders, R., Dowda, M., Felton, G., \& Pate, R.R. (2001). Measuring enjoyment of physical activity in adolescent girls. American Journal of Preventive Medicine, 21, 110-117.

National Association for Sport and Physical Education. (2004). Physical activity for children: A statement of guidelines for children ages 5-12 ( $2^{\text {nd }}$ ed.). Reston, VA: Author.

National Association for Sport and Physical Education. (2005). Physical education for lifelong fitness: The physical best teacher's guide ( $2^{\text {nd }}$ ed.). Champaign, IL: Human Kinetics.

National Center for Health Statistics. (2005). Health, United States, 2005 with chartbook on trends in the health of Americans. Hyattsville, Maryland. Retrieved April 30, 2006, from http://www.cdc.gov/nchs/hus.htm.

National Institute on Media and the Family (2001). Sixth annual video and computer report card. Retrieved April 30, 2006, from http://www.mediaandthefamily.org/research/vgrc/2001-2.shtml.

Nietfeld, J.L. \& Enders, C.K. (2003). An examination of student teacher beliefs: Interrelationships between hope, self-efficacy, goal-orientations, and beliefs about learning. Current Issues in Education, 6(5), 1-30.

Noble, B.J., \& Noble, J.M. (1998). Perceived exertion: The measurement. In J.L. Duda (Ed.), Advances in sport and exercise psychology measurement. (pp. 371390). Morgantown, WV: Fitness Information Technology. 
Pangrazi, R.P., Beighle, A., Vehige, T., \& Vack, C. (2003). Impact of promoting lifestyle activity for youth (PLAY) on children's physical activity. Journal of School Health, 73, 317-321.

Pate, R.R., Small, M.L., Ross, J.G., Young, J.C., Flint, K.H., \& Warren, C.W. (1995). School physical education. Journal of School Health, 65, 312-318.

Pietrobelli, A., Faith, M.S., Allison, D.B., Gallagher, D., Chiumello, G., \& Heymsfield, S.B. (1998). Body mass index as a measure of adiposity among children and adolescents: A validation study. Journal of Pediatrics, 132, 204-210.

Play to get fit - new concept? (2005, January 9). Retrieved January 10, 2005, from http://www.wired.com/news/print/0,1294,66219,00.html

Portman, P.A. (2003). Are physical education classes encouraging students to be physically active?: Experiences of ninth graders in their last semester of required physical education. Physical Educator, 150-161.

Prochaska, J.J., Sallis, J.F., Slymen, D.J., \& McKenzie, T.L. (2003). A longitudinal study of children's enjoyment of physical education. Pediatric Exercise Science, 15, $170-178$.

Provenzo, E.F. (1991). Video kids: Making sense of Nintendo. Cambridge, MA: Harvard University Press.

Rideout, V.J., Vandewater, E.A., \& Wartella, E.A. (2003). Zero to six: Electronic media in the lives of infants, toddlers and preschoolers. Retrieved February 5, 2005 from www.kff.org/content/2003/20031028

Ridley, K. \& Olds, T. (2001). Video center games: Energy cost and children's behaviors. Pediatric Exercise Science, 13, 413-421. 
Robertson, R.J. (2004). Perceived exertion for practitioners: Rating effort with the OMNI picture system. Champaign, IL: Human Kinetics.

Robertson, R.J., Goss, F.L., Andreacci, J., Dube, J.J., Rutkowski, J.J., Snee, B.M., Kowallis, R.A., Crawford, K., Aaron, D.J., \& Metz, K.F. (2005). Validation of the children's OMNI RPE scale for stepping exercise. Medicine and Science in Sports and Exercise, 37, 290-298.

Robertson, R.J., Goss, F.L., Boer, N.F., Peoples, J.A., Foreman, A.J., Dabayebeh, I.M., Millich, N.B., Balasekaran, G., Riechman, S.E., Gallagher, J.D. \& Thompkins, T. (2000). Children's OMNI scale of perceived exertion: Mixed gender and race validation. Medicine and Science in Sports and Exercise, 32, 452-458.

Robertson, R.J., \& Noble, B.J. (1997). Perception of physical exertion: Methods, mediators and applications. Exercise and Sport Sciences Reviews, 25, 407-452.

Robinson, T.N. (2001). Television viewing and childhood obesity. Pediatric Clinics of North America, 48, 1017-1025.

Ross, J.G., Pate, R.R., Corbin, C.B., Deply, L.A., \& Gold, R.S. (1987). What is going on in the elementary physical education program? Journal of Physical Education, Recreation and Dance, 58, 78-84.

Rowe, D.A., Mahar, M.T., Raedeke, T.D. \& Lore, J. (2004). Measuring physical activity in children with pedometers: Reliability, reactivity, and replacement of missing data. Pediatric Exercise Science, 16, 343-354.

Rowland, T.W. (1996). Developmental exercise physiology. Champaign, IL: Human Kinetics. 
Rowlands, A.V., Eston, R.G., \& Ingledew, D.K. (1999). Relationship between activity levels, aerobic fitness, and body fat in 8-10 yr-old children. Journal of Applied Physiology, 86, 1429-1435.

Ryan, G.J. \& Dzewaltowski, D.A. (2002). Comparing the relationships between different types of self-efficacy and physical activity in youth. Health Education and Behavior, 29, 491-504.

Sallis, J.F. \& McKenzie, T.L. (1991). Physical education's role in public health. Research Quarterly for Exercise and Sport, 62, 124-137.

Sallis, J.F., McKenzie, T.L., Alcaraz, J.E., Kolody, B., Faucette, N., \& Hovell, M.F. (1997). The effects of a 2-year physical education program (SPARK) on physical activity and fitness in elementary school students. American Journal of Public Health, 87, 1328-1334.

Sallis, J.F. \& Patrick, K. (1994). Physical activity guidelines for adolescents: Consensus statement. Pediatric Exercise Science, 6: 302-314.

Sallis, J.F., Prochaska, J.J., \& Taylor, W.C. (2000). A review of correlates of physical activity of children and adolescents. Medicine and Science in Sports and Exercise, 32, 963-975.

Sallis, J.F., Prochaska, J.J., Taylor, W.C., Hill, J.O., \& Geraci, J.C. (1999). Correlates of physical activity in a national sample of girls and boys in grades 4 through 12 . Health Psychology, 18, 410-415. 
Schmitt, M.J., Peterson, S.C., \& Cruz, L.M. (2003). Using heart rate monitors to compare the physical activity levels of males and females in coeducational and gender segregated sixth-grade physical education classes. [Abstract]. Journal of Physical Education, Recreation and Dance, 74, A54-A55.

Schneider, P.L., Crouter, S.E., Lukajic, O., \& Bassett, D.R., Jr. (2003). Accuracy and reliability of 10 pedometers for measuring steps over a $400-\mathrm{m}$ walk. Medicine and Science in Sports and Exercise, 35, 1779-1784.

Schnitzler, N. (2001, April 17). Dancing video game causes some Hawaii students to lose weight, money. Retrieved June 10, 2004, from http://www.ddrfreak.com/newpress/Reuters.htm

Scruggs, P.W., Beveridge, S.K., Watson, D.L., \& Clocksin, B.D. (2005) Quantifying physical activity in first- through fourth-grade physical education via pedometry. Research Quarterly for Exercise and Sport, 76, 166-175.

Segal, K. \& Dietz, W.H. (1991). Physiologic responses to playing a video game. American Journal of the Disabled Child, 145, 1034-1036.

Simons-Morton, B.G., McKenzie, T.J., Stone, E., Mitchell, P., Osganian, V., Strikmiller, P.K., Ehlinger, S., Cribb, P., \& Nader, P.R. (1997). Physical activity in a multiethnic population of third graders in four states. American Journal of Public Health, 87, 45-50.

Sirard, J.R. \& Pate, R.R. (2001). Physical activity assessment in children and adolescents. Sports Medicine, 31, 439-454. 
Sleap, M. \& Tolfrey, K. (2001). Do 9- to 12-yr-old children meet existing physical activity recommendations for health? Medicine and Science in Sports and Exercise, 33, 591-596.

Smith, B.K. (2005). Physical fitness in virtual worlds. Computer, 38, 101-103.

Steinbeck, K. (2001). Obesity in children - the importance of physical activity. Australian Jorunal of Nutrition and Dietetics, 58(Suppl 1), S28-S32.

Stewart, J.A., Dennison, D.A., Kohl, H.W., III, \& Doyle, J.A. (2004). Exercise level and energy expenditure in the TAKE 10 ! in-class physical activity program. Journal of School Health, 74, 397-400.

Tan, B., Aziz, A.R., Chua, K., \& Teh, K.C. (2002). Aerobic demands of the dance simulation game. International Journal of Sports Medicine, 23, 125-129.

Terbizan, D.J., Dolezal, B.A., \& Albano, C. (2002). Validity of seven commercially available heart rate monitors. Measurement in Physical Education and Exercise Science, 6, 243-247.

Thompson, L.P., Romanow, S.K.E., \& Horne, T.E. (1998). Factors influencing intentions and motor engagement behaviour of children in an instructional physical activity program. Avante, 4(1), 23-42.

Tremblay, M.S. \& Willms, J.D. (2003) Is the Canadian childhood obesity epidemic related to physical inactivity? International Journal of Obesity, 27, $1100-1105$.

Trost, S.G., Kerr, L.M., Ward, D.S., \& Pate, R.R. (2001). Physical activity and determinants of physical activity in obese and non-obese children. International Journal of Obesity, 25, 822-829. 
Trost, S.G., Pater, R.R., Sallis, J.F., Freedson, P.S., Taylor, W.C., Dowda, M., \& Sirard, J. (2002). Age and gender differences in objectively measured physical activity in youth. Medicine \& Science in Sports \& Exercise, 34(2), 350-355.

Trost, S.G., Pate, R.R., Saunders, R., Ward, D., Dowda, M., \& Felton, G. (1997). A prospective study of the determinants of physical activity in rural fifth grade children. Preventive Medicine, 26, 257-263.

Trudeau, F., \& Shephard, R.J. (2005). Contribution of school programmes to physical activity levels and attitudes in children and adults. Sports Medicine, 35, 89-105.

Tudor-Locke, C. \& Bassett, D.R., Jr. (2004). How many steps/day are enough? Preliminary pedometer indices for public health. Sports Medicine, 34, 1-8.

Tudor-Locke, C., Williams, J.E., Reis, J.P., \& Pluto, D. (2002). Utility of pedometers for assessing physical activity: Convergent validity. Sports Medicine, 32, 795-808.

Tudor-Locke, C., Williams, J.E., Reis, J.P., \& Pluto, D. (2004). Utility of pedometers for assessing physical activity: Construct Validity. Sports Medicine, 34, 281-291.

U.S. Department of Health and Human Services. (1996). Physical activity and health: A report of the Surgeon General. Atlanta: U.S. Department of Health and Human Services, Centers for Disease Control and Prevention, National Center for Chronic Disease Prevention and Health Promotion.

U.S. Department of Health and Human Services. (2000) Healthy People 2010: Understanding and Improving Health. 2nd ed. Washington, DC: U.S. Government Printing Office. 
Utter, A.C., Robertson, R.J., Nieman, D.C. \& Kang, J. (2002). Children's OMNI scale of perceived exertion: Walking/running evaluation. Medicine and Science in Sports and Exercise, 34, 139-144.

Vandewater, E.A., Shim, M., \& Caplovitz, A.G. (2004). Linking obesity and activity level with children's television and video game use. Journal of Adolescence, 27, 71-85.

Vincent, S.D., \& Pangrazi, R.P. (2002). An examination of the activity patterns of elementary school children. Pediatric Exercise Science, 14, 432-441.

Vincent, S.D., Pangrazi, R.P., Raustorp, A., Tomson, L.M., \& Cuddihy, T.F. (2003). Activity levels and body mass index of children in the United States, Sweden, and Australia. Medicine and Science in Sports and Exercise, 35, 1367-1373.

Welk, G.J. (1999). The youth physical activity promotion model: A conceptual bridge between theory and practice. Quest, 51, 5-23.

Williams, J.G., Eston, R., \& Furlong, B. (1994). CERT: A perceived exertion scale for young children. Perceptual and Motor Skills, 79, 1451-1458.

Winters, E.R., Petosa, R.L., \& Charlton, T.E. (2003). Using social cognitive theory to explain discretionary, "leisure-time" physical exercise among high school students. Journal of Adolescent Health, 32, 436-442.

Wood, D.A. (1986). Aerobic dance for children: Resources and recommendations. The Physician and Sportsmedicine, 14, 225-229. 
Woolfolk Hoy, A. (2000). Changes in teacher efficacy during the early years of teaching. Paper presented at the annual meeting of the American Educational Research Association, New Orleans, LA.

Yang, S.P. \& Graham, G.M. (2005, April). Project GAME (gaming activities for more exercise). Poster session presented at the annual Research Consortium of the American Alliance for Health, Physical Education, Recreation, and Dance (AAHPERD) National Convention, Chicago, Illinois.

Yelling, M., Lamb, K.L., \& Swaine, I.L. (2002). Validity of a pictorial perceived exertion scale for effort estimation and effort production during stepping exercise in adolescent children. European Physical Education Review, 8, 157-175. 


\section{Appendix A}

\section{Letter of Permission to Conduct Research}

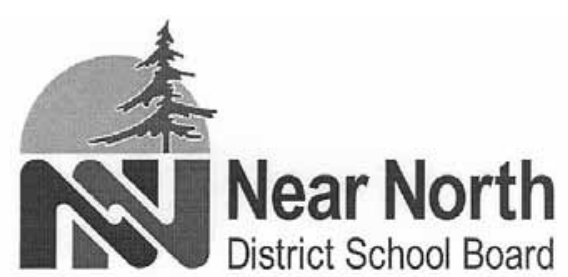

\begin{tabular}{l} 
Office \\
of the \\
Director of \\
Education \\
\hline P.O Box 3110 \\
963 Airport Road \\
North Bay, ON \\
P1B 8 H1 \\
Phone: \\
705.472.8170 \\
Toll Free: \\
1.800.278.4922 \\
Fax: 705.472 .9927 \\
Web Site: \\
www.nearnorth.edu.on.ca
\end{tabular}

April 4, 2005

Barbara Olmstead, B.A-B.P.H.E., B.Ed, M.A.

Nipissing University

P.O. Box 5002

North Bay, ON P1B 8L7

Dear Ms. Olmstead:

\section{RE: Doctoral Candidate at West Virginia University}

Recently, I was pleased to have the opportunity to meet with you and discuss your doctoral research proposal. I appreciate the information and "hands on" demonstration that you provided.

I am pleased to offer my permission to you to pursue your field work with junior level students in our elementary schools. I would ask that you provide the participating principals and me, when any of the research documentation as it relates to ethical matters such as the contact with students, prior to the publication of results.

I would like to offer my best wishes for success. I trust your research will engage and support our students as the pursue success in our schools.

Sincerely,

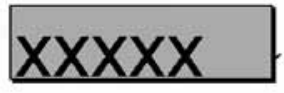

Colin Vickers

Director of Education

$\mathrm{CV}: \mathrm{cm}$ 


\section{Appendix B}

\section{Participant Information Sheet}

Name:

Grade:

Code Number:

Gender: $(\mathrm{M} / \mathrm{F}) \quad$ Height: (cm) Weight: $(\mathrm{kg})$

Age Range: 6-8 Years___ 9-11 Years___ 12+ Years

1. How active are you during the week? (not including gym class)

$\square \quad$ Not at all

$\square \quad$ 10-30 minutes per day

$\square \quad 30-60$ minutes per day

$\square \quad$ More than 60 minutes per day

2. How active are you on weekends?

$\square \quad$ Not at all

$\square \quad$ 10-30 minutes per day

$\square \quad 30-60$ minutes per day

$\square \quad$ More than 60 minutes per day

3. Have you ever played the video game Dance Dance Revolution (DDR)?

$\square \quad$ Never tried DDR

$\square \quad$ I have tried DDR once or twice

$\square \quad$ I have played DDR more than twice but not more than 10 times

$\square \quad$ I have played DDR quite a lot (more than 10 times)

$\square \quad$ I play DDR every day 


\section{Appendix C}

\section{West Virginia University IRB Approval}

Date: Mon, 05 Dec 2005 16:28:29 -0500

From: "Andrew Hawkins" <ahawkin2@wvu.edu>

Subject: Fwd: Re: IRB

To: "Barbara Olmsted" <barbarao@nipissingu.ca>

Barb,

In case there's another problem getting this from Lilo Ast.

Andy

>>> Lilo Ast 12/05/05 12:56 PM >>>

To: Barbara Olmsted

Thanks for your responses to our inquiries. The WVU IRB will accept the approval by the Nipissing IRB and it will not be necessary for you to file with us. You may proceed with your study according to the instructions you received from the Nipissing IRB. We will make a notation that your study has been approved by the Nipissing IRB, in case we need that information.

Sincerely,

Lilo Ast

Lilo Ast

Senior Program Coordinator

for Research Compliance

886 Chestnut Ridge Road

PO Box 6845

Telephone - 304-293-7555

Fax - 293-3098

Morgantown, WV 26508

Lilo.Ast@mail.wvu.edu

304-293-7435 (fax) 
Appendix D

Nipissing University Ethics Approval

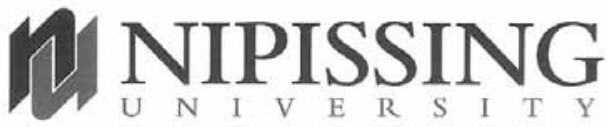

\section{NIPISSING UNIVERSITY ETHICS COMMITTEE}

ETHICAL REVIEW DECISION

Title: "The Effects of Interactive Video (DDR) on Heart Rate, Perceived Exertion, Selfefficacy, and Enjoyment in Elementary School Children"

Principal Researcher:

Barb Olmsted

Faculty/Department: Education

Date of Review Decision: June 22, 2005

The Ethical Review Committee has completed the examination of your research proposal. As Chair, it is my pleasure to inform you that your proposal meets all the requirements of the ethical review.

Best Wishes with your research

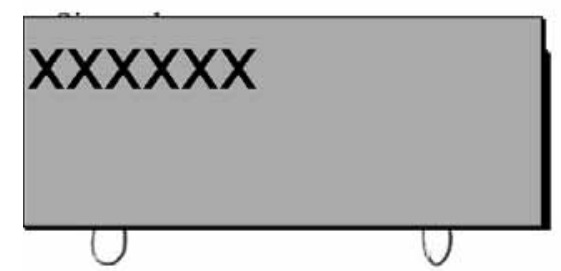

Dr. Steven High, Chair

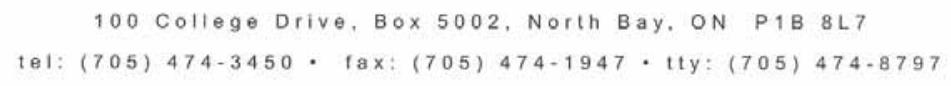




\section{Appendix E}

\section{Student Assent Form}

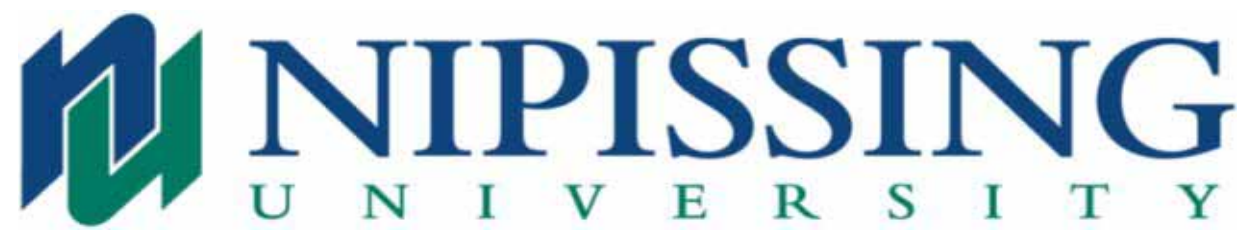

Dear Participant in the DDR Study:

This letter is to tell you about the activities you will participate in during this study. With your help, this research will help determine if DDR is a good activity for Physical Education classes. This study will measure a number of different things, including your heart rate, step counts, activity time, effort, enjoyment, and confidence. All of these things will be measured during 18 of your regular gym classes. You will be wearing a heart rate monitor attached to your ear lobe and a pedometer attached to your waistband. You will be asked to participate each gym day in one of three different activities that include DDR, aerobic dance and regular gym class. You won't know what is coming up each day, but you will be asked to do your best. Once you have finished each class, you will be asked to complete some questions about how you felt about the activity. These questions include how much you enjoyed the activity, how much effort you think you put into the activity, and how much confidence you have for continuing that activity outside class.

Once this data is collected, I will be sure that it is kept private. It will not be shared with anyone else, and your name will not be used for any purpose. I will keep all records locked in a file cabinet in my office.

Your participation in this study is voluntary, and you can decide to withdraw from the study at any time. If you decide not to participate, your grades will not be affected. If there are some questions you do not wish to answer, that is fine.

Thank you for your participation in the DDR Study. This study will help me complete my doctoral dissertation at West Virginia University. I hope that the information learned from this study will help students become more physically active in Physical Education classes.

Sincerely, Barbara Olmsted Doctoral Student Physical Education Teacher Education

Your signature indicates your informed consent to participate in this study. 


\section{Appendix F}

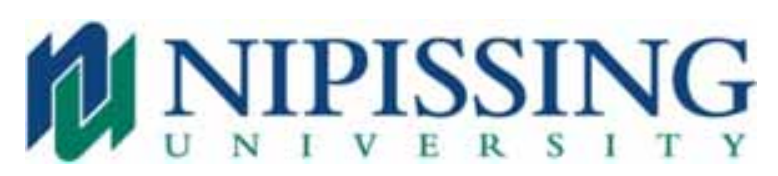

PARENTAL / GUARDIAN CONSENT FORM

\section{SIGNED CONSENT TO PARTICIPATE IN RESEARCH}

The Effects of Interactive Video (DDR) on Heart Rate, Perceived Exertion, Selfefficacy, and Enjoyment in Elementary School Children

Your child will be asked to participate in a research study conducted by Barbara Olmsted, from the Faculty of Education at Nipissing University. The results of the study will contribute to the completion of her doctoral dissertation at West Virginia University. Internal funding has been granted from Nipissing University.

If you have any questions or concerns about the research, please feel free to contact Barbara Olmsted at 474-3461, Extension 4393, or email at barbarao@nipissingu.ca

\section{- PURPOSE OF THE STUDY}

This study will investigate the impact of the video game "Dance Dance Revolution" (DDR) on elementary school children with respect to average heart rate, step count, activity time, perceived exertion, physical activity self-efficacy, and physical activity enjoyment. A secondary purpose will compare DDR on these variables to more traditional physical education activities (regular PE and aerobic dance).

\section{- PROCEDURES}

If you and your child volunteer to participate in this study, we would ask you to do the following things:

1. Attend the parent information evening to be held on

2. Sign this consent and return it to the school with your son or daughter (or at the parent information evening).

3. Your child will be involved in the following procedure: 


\section{Appendix F, continued}

This study will involve three different physical activities: Dance Dance Revolution (an interactive video game), aerobic dance, and regular PE. Each physical activity will be presented randomly to students over 18 Physical Education classes (6 sessions of each activity). During each class, students will wear a heart rate monitor attached to the ear lobe and a pedometer attached to the waistband. These instruments will collect heart rates, step counts, and active time during each class. The classroom teacher will lead each class for a total of 20 minutes of active time and 10 minutes of organizational time. After each class, students will be asked to complete three questionnaires. The first questionnaire asks students to identify their perceived level of exertion on a scale of 1 to 10. The second questionnaire asks students how much they enjoyed the activity, and the third questionnaire asks students about how confident they are in their ability to continue that particular activity outside of class (self-efficacy). Samples of all instruments will be made available at the parent meeting.

All activities will take place at the school. DDR will take place in a classroom, and the aerobic dance and regular PE will take place in the gymnasium. All students will participate in all activities. Students and parents will have access to their personal data following the completion of the study. Research findings will also be made available to parents and students upon request. A copy of the results will be given to Mr. Dunn for his records.

\section{- POTENTIAL RISKS AND DISCOMFORTS}

Because this study will occur during regular school hours in Physical Education class, all school rules and routines will continue to be in place. Your child's teacher will be leading each Physical Education class. Your child will be asked to wear a heart rate monitor (attached to his or her ear lobe) and a pedometer (attached to his or her waistband) for the duration of each PE class only. These instruments are comfortable and easy to use.

\section{- POTENTIAL BENEFITS TO SUBJECTS AND/OR TO SOCIETY}

Your child will be participating in different types of physical activities that can also be pursued outside the school environment. Each Physical Education class in this study is designed to keep your child continually active for 20 minutes, so it is anticipated that your son or daughter will benefit physically, psychologically and socially from his or her participation. It is hoped that your son or daughter will enjoy DDR, and want to continue doing DDR as a way to stay healthy and active.

It is anticipated that this study will determine whether or not DDR can be used in the Physical Education curriculum as an enjoyable activity for students. Additionally, it will expose teachers to alternative ways to get their students active, 


\section{Appendix F, continued}

and will enable them to make judgments about how practical DDR might be for use in schools.

- PAYMENT FOR PARTICIPATION

There will be no remuneration for your voluntary participation in this study.

- CONFIDENTIALITY

Any information that is obtained in connection with this study and that can be identified with you or your son or daughter will remain confidential. All data will be collected immediately following each Physical Education class, and stored in a locked cabinet in the researcher's office. Data will be destroyed following the release of study findings. Data may be released for publication, but anonymity will maintained.

\section{- PARTICIPATION AND WITHDRAWAL}

You can choose whether your child is to be in this study or not. If your child volunteers to be in this study, he/she may withdraw at any time without consequences of any kind. You may exercise the option of removing your child's data from the study. You and/or your child may also refuse to answer any questions you don't want to answer and still remain in the study. The investigator may withdraw your child from this research if circumstances arise which warrant doing so. Because this study will take place during regular Physical Education classes, and the described activities are appropriate for Physical Education, it is anticipated that your son or daughter will be able to participate. If there is any medical reason why your son or daughter should not participate, please contact Barbara Olmsted.

\section{- RIGHTS OF RESEARCH SUBJECTS}

You and/or your child may withdraw consent at any time and discontinue participation without penalty. This study has been reviewed and received ethics clearance through Nipissing University's Research Ethics Committee. If you have questions regarding your rights as a research subject, contact:

Research Ethics Co-ordinator

Telephone: 705-474-3461, \# 4558

Nipissing University

North Bay, Ontario P1B 8L7 
Appendix F, continued

- SIGNATURE OF RESEARCH SUBJECT/LEGAL REPRESENTATIVE

I understand the information provided for the study "The Effects of Interactive Video (DDR) on Heart Rate, Perceived Exertion, Self-efficacy, and Enjoyment in Elementary School Children" as described herein. My questions have been answered to my satisfaction, and I agree to my child participating in this study. I have been given a copy of this form.

Signature of Parent / Guardian Date

- SIGNATURE OF INVESTIGATOR

In my judgment, the subject is voluntarily and knowingly giving informed consent to participate in this research study. 


\section{Appendix G}

Teacher Lesson Plans

\section{Dance Dance Revolution Lesson Plan}

\section{Ontario Curriculum Expectations:}

\section{Grade 5:}

Stability Skills - transfer body weight in a variety of ways, using changes in direction and speed

Locomotion/Travelling Skills - perform a combination of locomotion/traveling movements, incorporating a variety of speeds, in relationship to objects or others Physical Activity - participate vigorously in all aspects of the program; describe the factors that motivate participation in daily physical activity and connect them to various activities

Physical Fitness - improve their fitness levels by participating in vigorous physical activities for sustained periods of time (e.g. ten to fifteen minutes)

\section{Grade 6:}

Locomotion/Travelling Skills - perform a combination of locomotion/traveling skills using equipment

Physical Activity - participate vigorously in all aspects of the program; describe the factors that motivate participation in daily physical activity and begin to consider them when making their own choices of physical activities Physical Fitness - improve their personal fitness levels by participating in vigorous physical activities for sustained periods of time (e.g. ten to fifteen minutes) 
Appendix G, continued

Pre-period Set Up:

- Classroom setting

- $6 \mathrm{TV} / \mathrm{X}$-Box carts, 24 dance pads

- Students can assist in set up

Student Preparation:

- As students enter the DDR room, they can pick up their pre-assigned pedometer, heart rate monitor, OMNI perceived exertion and enjoyment scale handout, and a pencil out of their envelopes

- Students will place heart rate monitors on ear lobes, and pedometers on their waistbands

- Students will remove shoes and move to one of the dance pads (groups of 4 can be determined by you, or self selected each session)

- Identify the student on pad \#1 (left side) as the one who selects the songs (position can be rotated each class)

Implementation:

- Ask students to turn on their heart rate monitors and zero their pedometers

- Ask students to set DDR to game mode choose their first song and

- Set a goal to complete at least 12 songs in 20 minutes (time this period)

- Adjust volume as necessary

- Encourage students as you normally would in physical education class

- Ask students to remember their best grade $(\mathrm{A}, \mathrm{B}, \mathrm{C}, \mathrm{D}, \mathrm{E})$ which will be recorded

- Do not participate with students unless you regularly participate in PE classes 
Appendix G, continued

Lesson Closure:

- Following 20 minutes of active time, ask students to stop their heart rate monitors and remove both the monitor and the pedometer, placing them into the envelope

- Give students time to complete the OMNI perceived exertion and enjoyment scale handout

- Have students put equipment away in designated area once pedometers and heart rate monitors have been returned to envelops and questionnaires completed

\section{Exercise Video (Tae-Bo) Lesson Plan:}

\section{Ontario Curriculum Expectations:}

\section{Grade 5:}

Stability Skills - transfer body weight in a variety of ways, using changes in direction and speed

Locomotion/Travelling Skills - perform a combination of locomotion/traveling movements, incorporating a variety of speeds, in relationship to objects or others Physical Activity - participate vigorously in all aspects of the program; describe the factors that motivate participation in daily physical activity and connect them to various activities

Physical Fitness - improve their fitness levels by participating in vigorous physical activities for sustained periods of time (e.g. ten to fifteen minutes)

\section{Grade 6:}

Locomotion/Travelling Skills - perform a combination of locomotion/traveling skills using equipment 
Appendix G, continued

Physical Activity - participate vigorously in all aspects of the program; describe the factors that motivate participation in daily physical activity and begin to consider them when making their own choices of physical activities Physical Fitness - improve their personal fitness levels by participating in vigorous physical activities for sustained periods of time (e.g. ten to fifteen minutes)

\section{Pre-period Set Up:}

- Gymnasium

- Move two TV's (one with DVD player) to gymnasium

- Connect the two televisions, insert aerobic dance DVD

Student Preparation:

- Students enter the gymnasium with appropriate footwear

- Students pick up their pre-assigned pedometer, heart rate monitor, OMNI perceived exertion and enjoyment scale handout, and a pencil out of their envelopes

- Students will place heart rate monitors on ear lobes, and pedometers on their waistbands

- Students spread out around the TV's

Implementation:

- Ask students to follow the DVD as closely as possible

- Encourage students as you normally would in PE class

- Time the lesson for a total of 20 minutes

- Participate if it is something you would normally do in PE class 
Appendix G, continued

Lesson Closure:

- After stopping the DVD, ask students to stop their heart rate monitors, place pedometers and heart rate monitors carefully back into the correct envelope, and complete and submit the OMNI perceived exertion and enjoyment scales

- Students return to classroom, equipment is returned to DDR room

\section{DDR INSTRUCTIONS FOR X-BOX}

Once you have the X-Box correctly hooked up to your TV, turn on the power, open the CD drive, and insert the DDR CD. Press close. The game should start automatically. Follow these steps to properly set up the game for your workout:

1. Press A when the instruction screen comes up

2. When the next screen comes up (SELECT MODE), use the UP or BACK arrow until OPTIONS lights up, press A

3. Use RIGHT arrow to scroll through until GAME lights up, press A

4. Use BACK arrow to go to EMPTY GAUGE END; use RIGHT arrow to select OFF

5. Use BACK arrow to DEFAULT DIFFICULTY; use RIGHT arrow to select BEGINNER

6. Press A to EXIT

7. Press B to go back to SELECT MODE, Press A

8. When the SELECT MODE screen comes up, press the BACK arrow until GAME MODE lights up, then press A. 
Appendix G, continued

9. Select SINGLE, press A.

10. Select SKILL LEVEL, press A.

11. Other players must press A on their pads to join.

12. Select MUSIC by pressing RIGHT arrow 2 times until the song "BRICK HOUSE" shows in the box, press A.

13. After the song, the word CLEARED comes across the screen, and then the GRADE screen is displayed showing your grade (A, B, C, D, or E).

14. Press A to continue

15. Select the next song, press A

16. Continue this process until you have completed all of your required songs.

17. If you need to re-start the game at any time, press and hold START.

18. If you need to go back to a previous screen, press B.

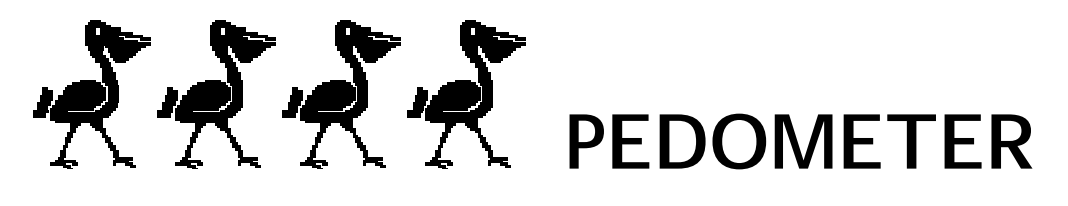

PO I $\mathcal{N} \mathcal{T} R S \ldots$

T. Wear your pedometer every gym class!

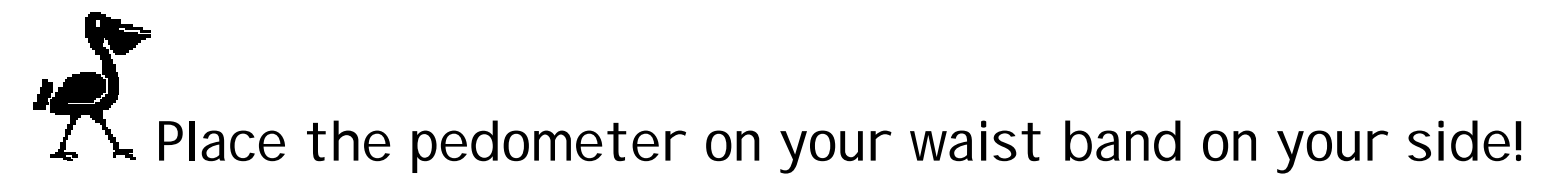


Appendix G, continued

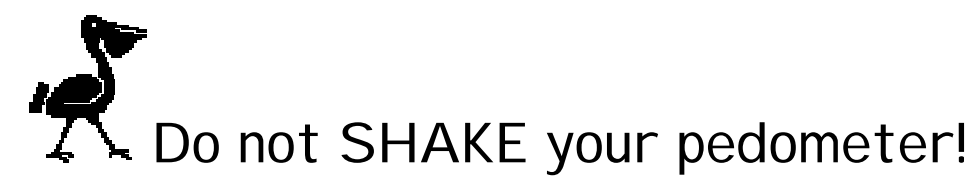

4 Press RES ET before you start your ac tivity!
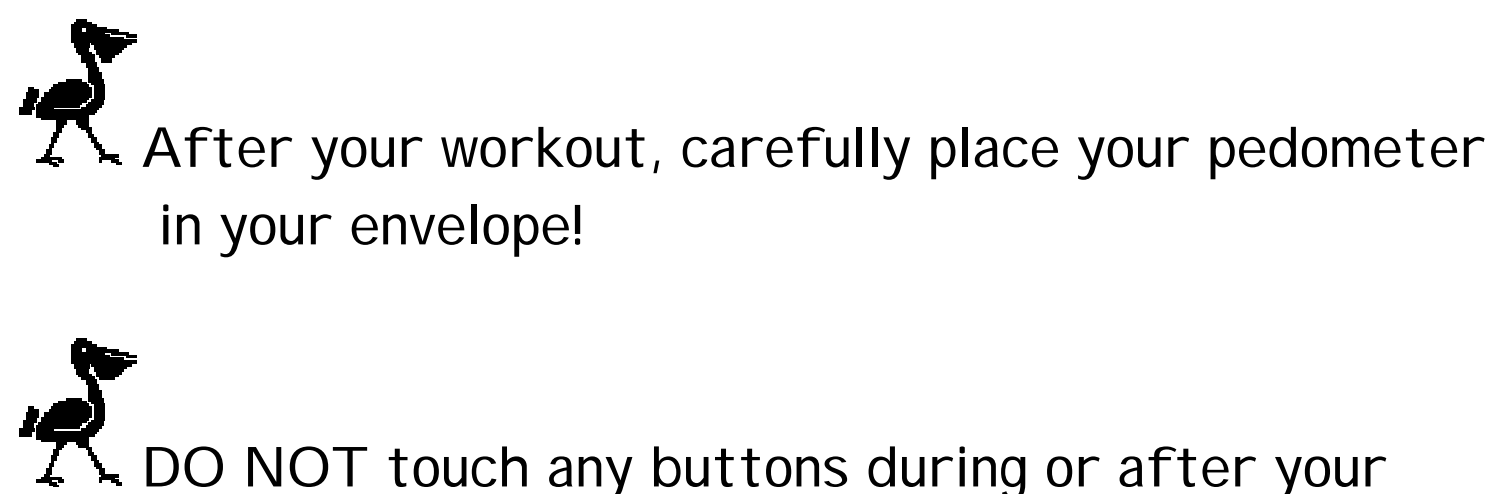

DO $\mathcal{N O T}$ touch any buttons during or after your workout!

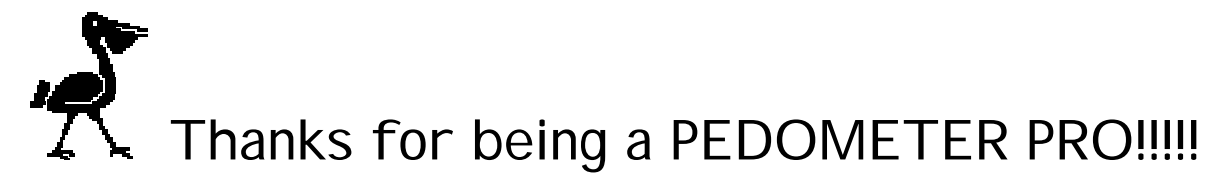

\section{Using the CATEYE PL-6000 Heart Rate Monitor}

1. Clip the earlobe sensor to the center of either earlobe. (Earrings must be taken off).

2. Attach the cable holder to the collar of your clothes to keep the wire from swinging too much.

3. Attach the main unit to your waist band or upper arm using the strap provided.

4. Press the ADV button once. The display should appear. 
Appendix G, continued

5. When you are about to begin your exercise session, press the ADV button once; the unit starts counting the elapsed time, and the walking man starts blinking.

6. DO NOT TOUCH the unit again until the end of your session.

7. Once you have completed your exercise, press MEMO once, and ADV once.

8. Remove the heart rate monitor, leaving it $\mathrm{ON}$.

9. Place the heart rate monitor carefully in your envelope.

10. To retrieve the average heart rate for the session, hole the MODE button down over 2 seconds to turn the screen to RECALL DISPLAY.

11. The first memory data M1 is displayed. This is the average pulse rate for that exercise session, and this number will be recorded. 
Appendix G, continued

\section{J.W. Trusler Public School Near North Board of Education}

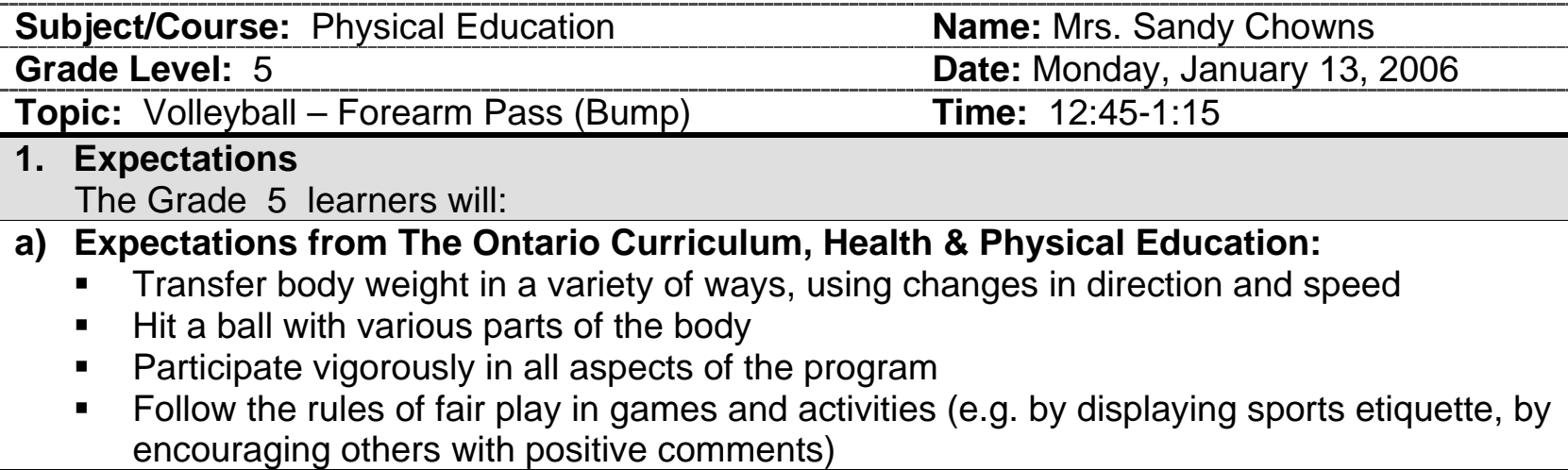

2. Preassessment

a) Learners:

- 22 students, 5 with IEP's (special needs)

- 6 students on the school volleyball team

- Previous experience with volleyball in grade 4 included volleying, bumping and serving

- Previous lessons in grade 5 involved review of above skills

b) Learning Environment:

- Single gym with stage at one end (size of full volleyball court)

- Two nets set up prior to the start of class, forming two small courts

c) Resources:

- 12 volleyballs (may need to inflate some prior to class)

- 2 nets

- Whistle

3. Content

Forearm Pass (Bump)

- Stance - feet shoulder width or wider, weight forward (bent at waist), knees bent (should not be able to see feet)

- Hand position - two ways - fist with one hand, wrapping other hand around the fist, press thumbs down, OR, one hand palm up, place second hand palm up within it, thumbs together and pressed down

- Arm position - elbows straight, arms away from body (45 degree angle), shoulders pressed forward

- Contact point - just above the wrist (watchband) - BUNT the ball

- Prior to contact - move body behind ball, face the target, CALL the ball

- Arm action at contact - wait for the ball, DO NOT SWING ARMS to meet the ball, use legs to bunt the ball

- Get back into ready position with arms in front of body, eyes up

Game Play

- Call the ball

- Attempt 3 contacts per side

- Use positive comments to team mates and opponents - ALWAYS

- King's Court Game - teams of three, lined up behind the baseline; first two teams play out the rally, winner goes to the King's Court side; loser retrieves the ball and joins the 


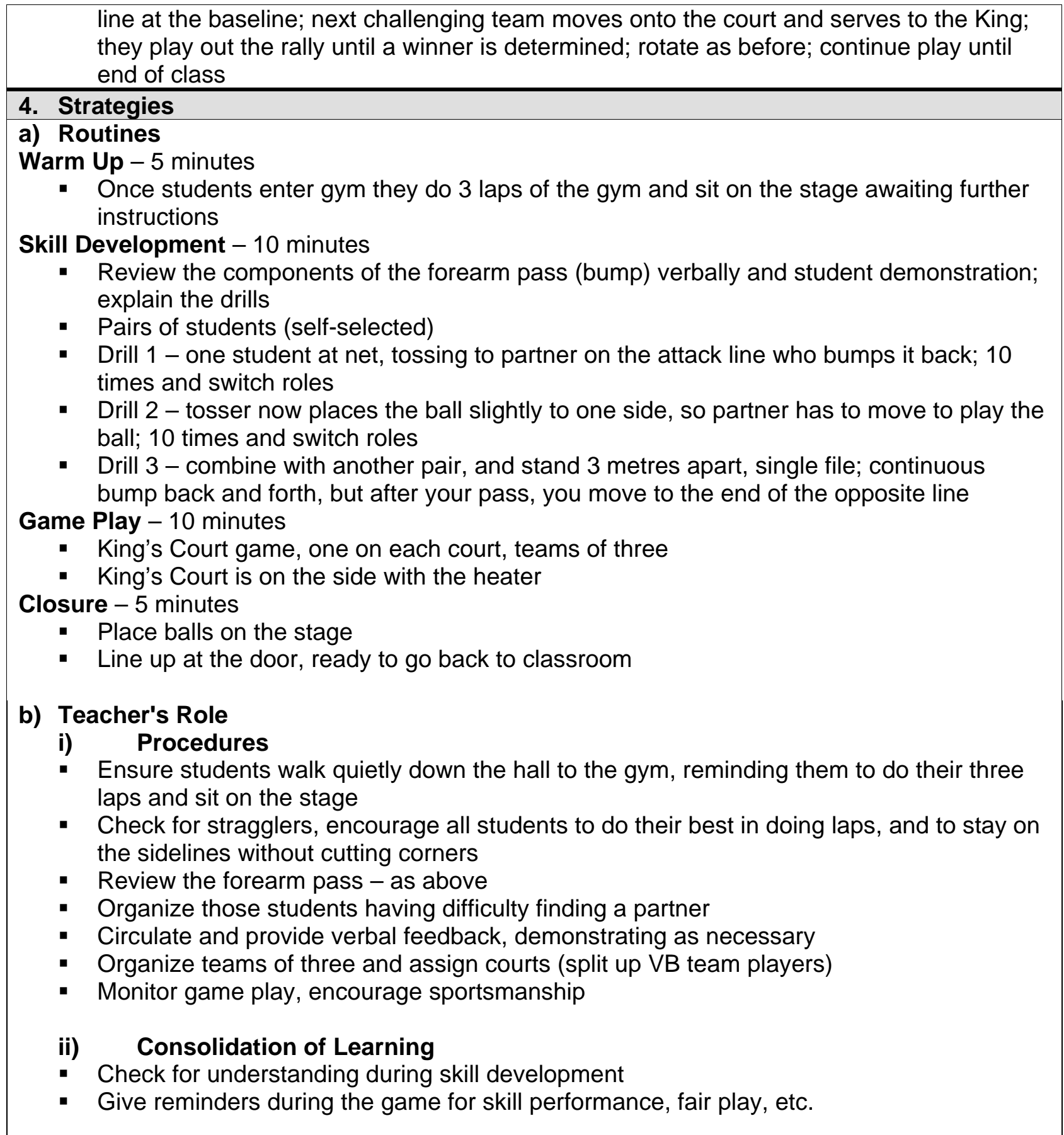

\section{Assessment}

- Active participation rubric

- Anecdotal notes and observations regarding skill development

6. Reflections

a) Learning Expectations

\section{b) Effectiveness}


Appendix G, continued

J.W. Trusler Public School

Activity Planning Format

Near North Board of Education

\begin{tabular}{ll} 
Subject/Course: Physical Education & Name: Mrs. Sandy Chowns \\
\hline Grade Level: 5 & Date: Monday, January 20, 2006 \\
\hline Topic: Volleyball - Forearm Pass (Bump), Overhead & Time: 12:45-1:15 \\
Volley & \\
\hline
\end{tabular}

1. Expectations

The Grade 5 learners will:

b) Expectations from The Ontario Curriculum, Health \& Physical Education:

- Transfer body weight in a variety of ways, using changes in direction and speed

- Hit a ball with various parts of the body

- Participate vigorously in all aspects of the program

- Follow the rules of fair play in games and activities (e.g. by displaying sports etiquette, by encouraging others with positive comments)

\section{Preassessment}

d) Learners:

- 22 students, 5 with IEP's (special needs)

- 6 students on the school volleyball team

- Previous experience with volleyball in grade 4 included volleying, bumping and serving

- Previous lessons in grade 5 involved review of above skills

- Have already worked on forearm passing and ready position

e) Learning Environment:

- Single gym with stage at one end (size of full volleyball court)

- One net set up prior to the start of class

f) Resources:

- 24 volleyballs (may need to inflate some prior to class)

- 1 nets

- Whistle

3. Content

Forearm Pass (Bump)

- Stance - feet shoulder width or wider, weight forward (bent at waist), knees bent (should not be able to see feet)

- Hand position - two ways - fist with one hand, wrapping other hand around the fist, press thumbs down, OR, one hand palm up, place second hand palm up within it, thumbs together and pressed down

- Arm position - elbows straight, arms away from body (45 degree angle), shoulders pressed forward

- Contact point - just above the wrist (watchband) - BUNT the ball

- Prior to contact - move body behind ball, face the target, CALL the ball

- Arm action at contact - wait for the ball, DO NOT SWING ARMS to meet the ball, use legs to bunt the ball

- Get back into ready position with arms in front of body, eyes up Overhead Volley

- Stance - feet shoulder width or narrower, weight forward (straighter at waist), knees slightly bent

- Hand position - form triangle with thumbs and forefinger, finders spread but curved; use the pop bottle analogy (e.g. pretend to use both hands to raise a large pop bottle up to 


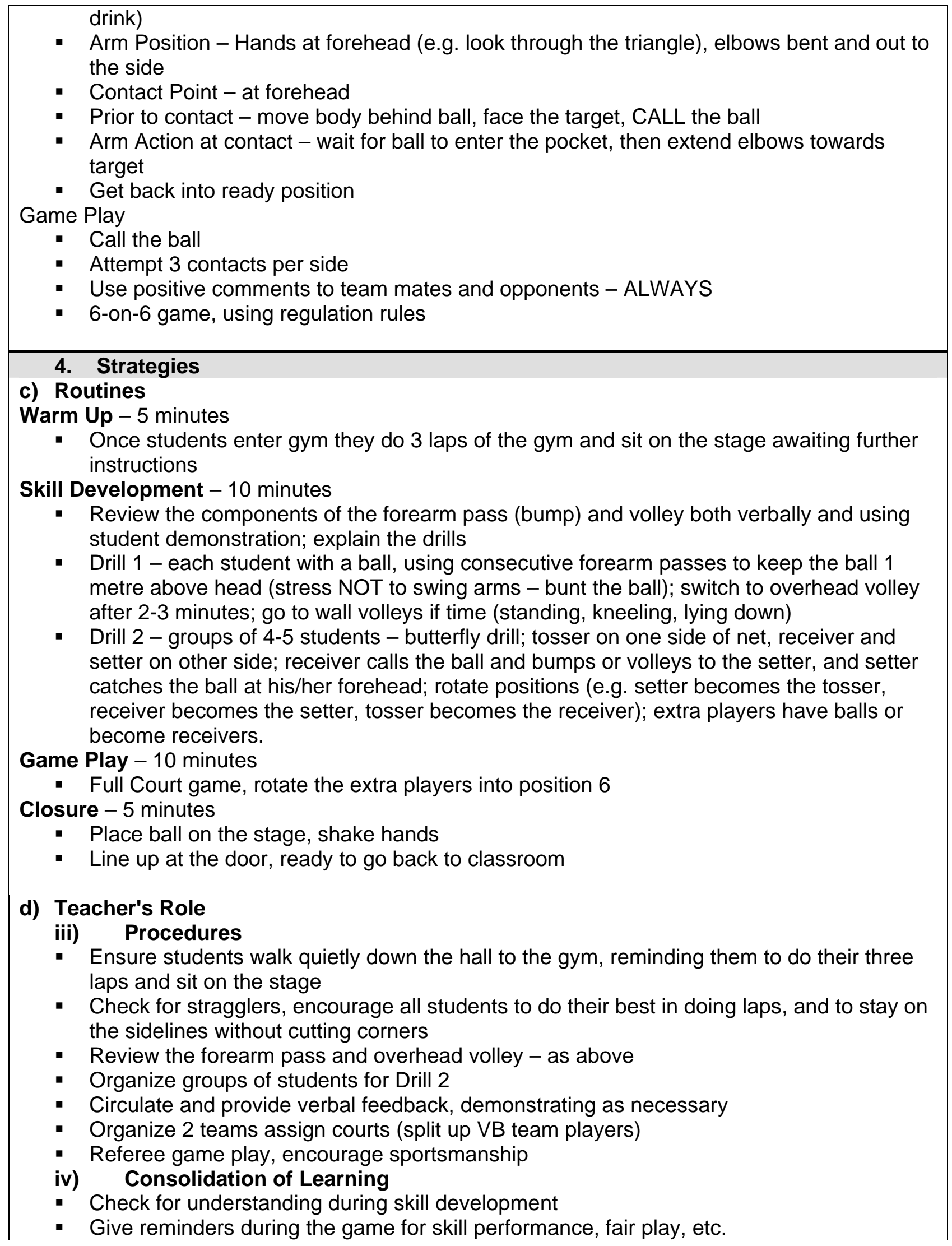


Interactive Video 173

5. Assessment

- Active participation rubric

- Skill development rubric

6. Reflections

c) Learning Expectations

d) Effectiveness 
Appendix $\mathrm{H}$

Permission for Use of OMNI Perceived Exertion Scale

Dear Ms. Olmsted:

I was pleased to learn of your interest in perceived exertion research. Your dissertation topic will make a significant contribution to the applied dimensions of the perceived exertion knowledge base.

One particular advantage of the Children's OMNI Scale is that it employs interchangeable sets of exercise specific pictorial descriptors that are used with a standard core format. If your exercise program employs separate sets of stepping and running, I suggest that the step and walk/run pictorial sets be used according to the appropriate exercise station. If this is not possible in the school setting, then either one of the two pictorial sets can be applied without losing scale validating. Recent research from our laboratory (Robertson, R, et al. MSSE, 37, 2, 290-298, 2005) indicates that the gender depicted by the pictorial set does not present a systematic bias in OMNI Scale RPE. As such either or both gender specific stepping pictorial sets can be employed with children.

If I can be of further assistance, please contact me. Good luck with your dissertation research.

Sincerely,

Robert J. Robertson, Ph.D.

Professor and Co-Director

Center for Exercise and Health-Fitness Research

--On Sunday, April 03, 2005 2:22 PM -0400 Barbara Joan Olmsted

$<$ bolmsted@mix.wvu.edu>wrote:r

Hello Dr. Robertson:

I am a doctoral student in Physical Education Teacher Education at West Virginia University. I am in the process of preparing my dissertation proposal, and I wish to seek your permission to use the OMNI RPE Scale as one component in my study.

My study involves examining the impact of the interactive video game Dance Dance Revolution (DDR) on heart rate, RPE, enjoyment and self-efficacy in elementary school children (grades 4-6). I am hoping to use the school setting with an alternating treatment design for 
classes within the school. DDR will be one treatment, and I will compare that to fitness stations and aerobics. Students will be doing a combination of stepping and running during each treatment.

Perhaps you could recommend which scale I should use - stepping scale or running scale, and whether or not I should make it gender specific or gender neutral.

I would appreciate any feedback you could provide. In addition, if you could attach to your email a brief letter of permission, and the appropriate scale you feel I should use, I would certainly be thankful.

Please do not hesitate to contact me should you require further information about my study.

My address and phone:

Barbara Olmsted

School of Physical Education

287 Coliseum

P.O. Box 6116

MOrgantown, WV 26506-6116

304-293-3295 Ext. 5290

I look forward to your response,

Barbara 


\section{OMNI Perceived Exertion Scale}

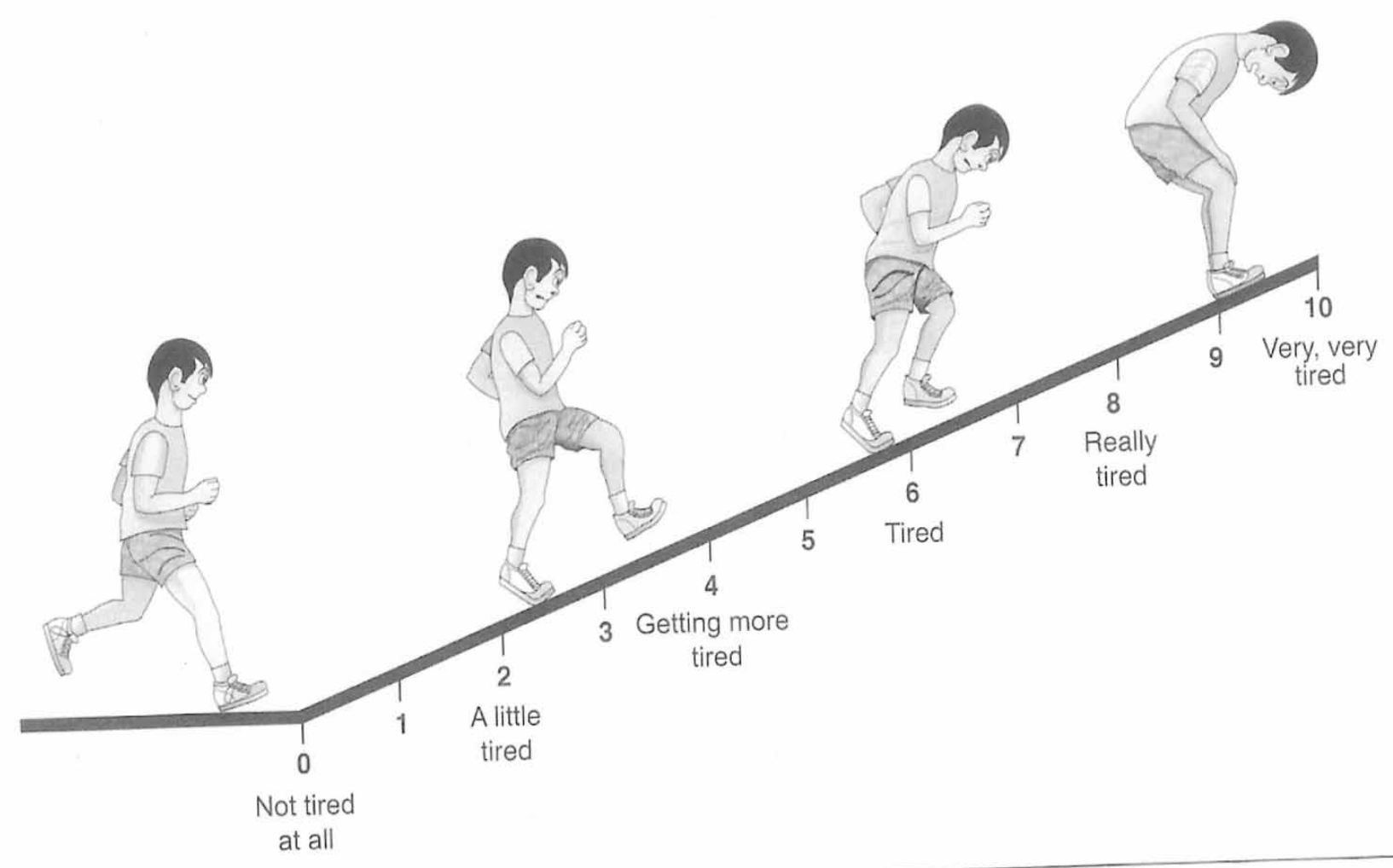

0
3
$Z$
0
0
0
0
0
0
0
0
0
0
0
0
0
0
0
0
0.0
0

OMNI Scale of Perceived Exertion: Child, Walking to Running Format

The OMNI Picture System by R.J. Robertson. Champaign, IL: Human Kinetics, 2004

(Reprinted with permission from Robertson, 2005) 


\section{Appendix J}

Permission for Use of Self-Efficacy Scale and Enjoyment Scale

\section{Barbara,}

You have our permission to use the scales. The files are attached. I've also attached a paper in press in the Am J Prev Med showing the scales have validity for 6th grade girls.

Good luck

Rod K. Dishman

Exercise Science

Department of Kinesiology

The University of Georgia

115 I Ramsey Center, 300 River Road

Athens, Georgia 30602-6554 USA

706-542-9840 phone

706-542-3148 fax

---- Original message ----

Date: Sun, 3 Apr 2005 14:54:47 -0400 (EDT)

From: Barbara Joan Olmsted <bolmsted@mix.wvu.edu $>$

Subject: Enjoyment Scale Permission

To: rdishman@coe.uga.edu

Hello Dr. Dishman,

I am a doctoral student in Physical Education Teacher

Education at West Virginia University. I am in the process of preparing my dissertation propsal, and I am writing to you to seek permission to use two measurement tools you have recently reported using in your research; the Enjoyment Scale as modified from PACES, and the 8-item measure of self-efficacy.

My study will involve school children in 4th-6th grade. I am interested in examining the effect of the interactive video game "Dance Dance Revolution" (DDR) on heart rate, RPE, enjoyment and self-efficacy. I plan to compare DDR to fitness station PE lessons and aerobics PE lessons.

I recognize that your scales may be too difficult for this age group to understand, but I hope to make them age appropriate through 
pilot work. Perhaps you have already explored this possibility.

I would be very appreciative if you could send me a letter of

permission, as well as a copy of the two scales. I would

also welcome any suggestions or feedback you may have with regard to my study.

I can be reached at:

Barbara Olmsted

287 Coliseum

School of Physical Education

P.O. Box 6116

Morgantown, WV 26506-6116

304-293-3295, ext. 5290

I look forward to hearing from you.

Barbara 


\section{Appendix K}

\section{Self-Efficacy Scale}

For each statement below, select the response which best represents how much you 'disagree' or 'agree' with the statement. Mark your response by placing an $\mathbf{X}$ or a $\sqrt{ }$ in the correct column.

\begin{tabular}{|c|c|c|c|c|c|}
\hline & $\begin{array}{l}\text { Disagree } \\
\text { a lot }\end{array}$ & $\begin{array}{l}\text { Disagree } \\
\text { a little }\end{array}$ & $\begin{array}{l}\text { Neither } \\
\text { agree nor } \\
\text { disagree }\end{array}$ & $\begin{array}{l}\text { Agree a } \\
\text { little }\end{array}$ & $\begin{array}{l}\text { Agree a } \\
\text { lot }\end{array}$ \\
\hline $\begin{array}{l}\text { 1. I can be physically active during my } \\
\text { free time on most days. }\end{array}$ & & & & & \\
\hline $\begin{array}{l}\text { 2. I can ask my parent or other adult to } \\
\text { do physically active things with me. }\end{array}$ & & & & & \\
\hline $\begin{array}{l}\text { 3. I can be physically active during my } \\
\text { free time on most days even if I could } \\
\text { watch TV or play video games instead. }\end{array}$ & & & & & \\
\hline $\begin{array}{l}\text { 4. I can be physically active during my } \\
\text { free time on most days even if it is very } \\
\text { hot or cold outside. }\end{array}$ & & & & & \\
\hline $\begin{array}{l}\text { 5. I can ask my best friend to be } \\
\text { physically active with me during my free } \\
\text { time on most days. }\end{array}$ & & & & & \\
\hline $\begin{array}{l}\text { 6. I can be physically active during my } \\
\text { free time on most days even if I have a } \\
\text { lot of homework. }\end{array}$ & & & & & \\
\hline $\begin{array}{l}\text { 7. I have the coordination I need to be } \\
\text { physically active during my free time on } \\
\text { most days. }\end{array}$ & & & & & \\
\hline $\begin{array}{l}8 . \text { I can be physically active during my } \\
\text { free time on most days no matter how } \\
\text { busy my day is. }\end{array}$ & & & & & \\
\hline
\end{tabular}


Appendix L

Physical Activity Enjoyment Scale

For each statement below, select the response which best represents how much you 'disagree' or 'agree' with the statement. Mark your response by placing an $\mathbf{X}$ or a $\sqrt{\text { in }}$ the correct column.

\begin{tabular}{|c|c|c|c|c|c|}
\hline When I am active ... & $\begin{array}{l}\text { Disagree } \\
\text { a lot }\end{array}$ & $\begin{array}{l}\text { Disagree } \\
\text { a little }\end{array}$ & $\begin{array}{l}\text { Neither } \\
\text { agree nor } \\
\text { disagree }\end{array}$ & $\begin{array}{l}\text { Agree a } \\
\text { little }\end{array}$ & $\begin{array}{l}\text { Agree a } \\
\text { lot }\end{array}$ \\
\hline 1. ... I feel bored. & & & & & \\
\hline 2. ... I dislike it. & & & & & \\
\hline 3. ... it's not fun at all. & & & & & \\
\hline 4. ... it makes me depressed. & & & & & \\
\hline $5 \ldots$ it frustrates me. & & & & & \\
\hline $6 \ldots$ it's not at all interesting. & & & & & \\
\hline $\begin{array}{l}\text { 7. ... I feel as though I would } \\
\text { rather be doing something } \\
\text { else. }\end{array}$ & & & & & \\
\hline
\end{tabular}




\section{Appendix M}

\section{Letter of Permission for Youth Physical Activity Promotion Model}

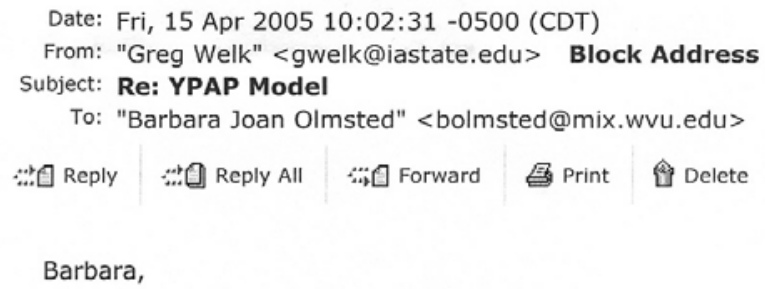




\section{Appendix N}

Mean Scores per Trial for Volleyball, Tae-Bo, and DDR

Table N1 Mean Heart Rate per Trial for Volleyball, Tae-Bo, and DDR

\begin{tabular}{cccccc}
\hline $\begin{array}{c}\text { Trial for } \\
\text { Activity Type }\end{array}$ & $\mathrm{N}$ & Minimum & Maximum & Mean & Std. Deviation \\
\hline hr_vb_t1 & 33 & 78.00 & 154.00 & 118.9091 & 19.83898 \\
hr_vb_t2 & 33 & 81.00 & 153.00 & 121.4545 & 19.40214 \\
hr_vb_t3 & 33 & 86.00 & 144.00 & 121.9091 & 12.42569 \\
hr_vb_t4 & 33 & 86.00 & 148.00 & 116.8485 & 14.59692 \\
hr_vb_t5 & 33 & 84.00 & 148.00 & 123.6667 & 15.52350 \\
hr_vb_t6 & 33 & 100.00 & 147.00 & 124.8182 & 13.98315 \\
hr_tb_t1 & 33 & 85.00 & 148.00 & 125.2424 & 18.20033 \\
hr_tb_t2 & 33 & 86.00 & 153.00 & 124.3636 & 15.92525 \\
hr_tb_t3 & 33 & 80.00 & 148.00 & 125.3939 & 19.15225 \\
hr_tb_t4 & 33 & 81.00 & 170.00 & 127.8182 & 21.69455 \\
hr_tb_t5 & 33 & 82.00 & 159.00 & 126.7879 & 18.29747 \\
hr_tb_t6 & 33 & 86.00 & 157.00 & 123.9091 & 17.85246 \\
hr_dr_t1 & 33 & 100.00 & 166.00 & 127.8182 & 16.90313 \\
hr_dr_t2 & 33 & 84.00 & 183.00 & 133.8182 & 18.54126 \\
hr_dr_t3 & 33 & 97.00 & 158.00 & 128.0303 & 15.23664 \\
hr_dr_t4 & 33 & 89.00 & 162.00 & 129.8788 & 17.74253 \\
hr_dr_t5 & 33 & 89.00 & 176.00 & 128.4848 & 17.75197 \\
hr_dr_t6 & 33 & 88.00 & 173.00 & 124.9697 & 20.23531
\end{tabular}

Table N2 Mean Step Count per Trial for Volleyball, Tae-Bo, and DDR

Trial for

\begin{tabular}{lccccc} 
Activity Type & N & Minimum & Maximum & Mean & Std. Deviation \\
\hline stp_vb_t1 & 33 & 217.00 & 2156.00 & 919.1818 & 440.63459 \\
stp_vb_t2 & 33 & 201.00 & 2362.00 & 1315.5455 & 514.83481 \\
stp_vb_t3 & 33 & 227.00 & 2015.00 & 1184.8788 & 393.92478 \\
stp_vb_t4 & 33 & 157.00 & 1522.00 & 818.7879 & 362.49912 \\
stp_vb_t5 & 33 & 349.00 & 1890.00 & 1158.3030 & 402.23264 \\
stp_vb_t6 & 33 & 210.00 & 2024.00 & 1228.3939 & 485.38869 \\
stp_tb_t1 & 33 & 314.00 & 2231.00 & 1299.5758 & 518.04784 \\
stp_tb_t2 & 33 & 356.00 & 1970.00 & 1187.6061 & 413.68670 \\
stp_tb_t3 & 33 & 506.00 & 2169.00 & 1204.3939 & 439.86482 \\
stp_tb_t4 & 33 & 211.00 & 2408.00 & 1341.8485 & 481.49034 \\
stp_tb_t5 & 33 & 402.00 & 1942.00 & 1276.3636 & 423.90593 \\
stp_tb_t6 & 33 & 376.00 & 2095.00 & 1265.2727 & 465.93772 \\
stp_dr_t1 & 33 & 253.00 & 2190.00 & 1279.0303 & 482.70491 \\
stp_dr_t2 & 33 & 823.00 & 2387.00 & 1604.2424 & 383.26998 \\
stp_dr_t3 & 33 & 555.00 & 2315.00 & 1439.0606 & 433.28382 \\
stp_dr_t4 & 33 & 676.00 & 2487.00 & 1509.4242 & 457.44119 \\
stp_dr_t5 & 33 & 550.00 & 2677.00 & 1591.1515 & 435.20018 \\
stp_dr_t6 & 33 & 481.00 & 2729.00 & 1521.3636 & 568.30866
\end{tabular}


Table N3 Mean Active Time (seconds) per Trial for Volleyball, Tae-Bo, and DDR

\begin{tabular}{cccccc}
\hline Trial for & & & & & \\
Activity Type & $\mathrm{N}$ & Minimum & Maximum & Mean & Std. Deviation \\
\hline at_vb_t1 & 33 & 267.00 & 1417.00 & 733.5152 & 282.37454 \\
at_vb_t2 & 33 & 257.00 & 1737.00 & 974.9091 & 345.51125 \\
at_vb_t3 & 33 & 254.00 & 1382.00 & 897.3333 & 252.99415 \\
at_vb_t4 & 33 & 112.00 & 1291.00 & 697.5758 & 284.27441 \\
at_vb_t5 & 33 & 351.00 & 1403.00 & 894.5758 & 251.80002 \\
at_vb_t6 & 33 & 234.00 & 1355.00 & 893.2727 & 285.66636 \\
at_tb_t1 & 33 & 271.00 & 1651.00 & 912.6061 & 264.92982 \\
at_tb_t2 & 33 & 380.00 & 1196.00 & 830.0909 & 204.13558 \\
at_tb_t3 & 33 & 417.00 & 1415.00 & 860.6364 & 236.30923 \\
at_tb_t4 & 33 & 266.00 & 1440.00 & 939.2121 & 248.62594 \\
at_tb_t5 & 33 & 437.00 & 1207.00 & 939.3333 & 199.37726 \\
at_tb_t6 & 33 & 338.00 & 1279.00 & 942.5455 & 244.38099 \\
at_dr_t1 & 33 & 335.00 & 1326.00 & 874.0909 & 230.83982 \\
at_dr_t2 & 33 & 784.00 & 1539.00 & 1045.7879 & 147.45757 \\
at_dr_t3 & 33 & 505.00 & 1542.00 & 988.8485 & 187.41100 \\
at_dr_t4 & 33 & 626.00 & 1349.00 & 1019.5455 & 202.61495 \\
at_dr_t5 & 33 & 146.00 & 1770.00 & 1054.1515 & 280.22091 \\
at_dr_t6 & 33 & 491.00 & 1526.00 & 1045.4242 & 248.77098
\end{tabular}

Table N4 Mean Elapsed Time per Trial for Volleyball, Tae-Bo and DDR

\begin{tabular}{|c|c|c|c|c|c|}
\hline $\begin{array}{c}\text { Trial for } \\
\text { Activity Type }\end{array}$ & $\mathrm{N}$ & Minimum & Maximum & Mean & Std. Deviation \\
\hline tt_vb_t1 & 33 & 1083.00 & 1699.00 & 1427.0000 & 233.13301 \\
\hline $\mathrm{tt} \mathrm{vb} \mathrm{t} 2$ & 33 & 1333.00 & 1781.00 & 1626.3333 & 96.11083 \\
\hline $\mathrm{tt}-\mathrm{vb}$ - $\mathrm{t} 3$ & 33 & 1281.00 & 1828.00 & 1576.7879 & 125.66716 \\
\hline $\mathrm{tt} \_\mathrm{vb} \_\mathrm{t} 4$ & 33 & 1013.00 & 1908.00 & 1721.4848 & 161.58069 \\
\hline $\mathrm{tt}-\mathrm{vb}$-t5 & 33 & 1167.00 & 1936.00 & 1560.2121 & 115.10152 \\
\hline $\mathrm{tt} \_\mathrm{vb} \mathrm{t}^{-} 6$ & 33 & 975.00 & 1968.00 & 1550.8182 & 248.07212 \\
\hline $\mathrm{tt} \_\mathrm{tb}-\mathrm{t} 1$ & 33 & 906.00 & 1443.00 & 1305.2424 & 127.73753 \\
\hline $\mathrm{tt}+\mathrm{tb} \mathrm{t}^{-} \mathrm{2}$ & 33 & 1047.00 & 1291.00 & 1204.7273 & 63.16411 \\
\hline $\mathrm{tt}$ tb $\mathrm{t} 3$ & 33 & 1122.00 & 1475.00 & 1265.5152 & 80.15147 \\
\hline $\mathrm{tt}^{-} \mathrm{tb} \mathrm{t}^{-}$ & 33 & 952.00 & 1556.00 & 1277.7576 & 114.86651 \\
\hline $\mathrm{tt}^{-} \mathrm{tb} \mathrm{t}^{-}$ & 33 & 808.00 & 1500.00 & 1323.0606 & 136.10541 \\
\hline $\mathrm{tt} \_\mathrm{tb} \_\mathrm{t} 6$ & 33 & 1260.00 & 1777.00 & 1384.7879 & 89.20368 \\
\hline $\mathrm{tt} \_\mathrm{dr} \_\mathrm{t} 1$ & 33 & 1040.00 & 1638.00 & 1330.6970 & 132.90774 \\
\hline $\mathrm{tt} \mathrm{dr} \mathrm{t} 2$ & 33 & 1393.00 & 1674.00 & 1483.9091 & 68.80968 \\
\hline $\mathrm{tt} \_\mathrm{dr} \mathrm{t} 3$ & 33 & 1236.00 & 1823.00 & 1516.9697 & 105.40828 \\
\hline $\mathrm{tt} \mathrm{dr} \mathrm{t} 4$ & 33 & 1161.00 & 1989.00 & 1555.7273 & 131.08901 \\
\hline $\mathrm{tt} \_\mathrm{dr}$ - $\mathrm{t} 5$ & 33 & 561.00 & 1648.00 & 1413.9394 & 184.60243 \\
\hline $\mathrm{tt}{ }^{-} \mathrm{dr}{ }^{-} \mathrm{t} 6$ & 33 & 1335.00 & 1940.00 & 1553.9394 & 145.30936 \\
\hline
\end{tabular}


Table N5 Mean Percent Active Time per Trial for Volleyball, Tae-Bo, and DDR

\begin{tabular}{cccccc}
\hline Trial for & & & & & \\
Activity Type & $\mathrm{N}$ & Minimum & Maximum & Mean & Std. Deviation \\
\hline ap_vb_t1 & 33 & 18.69 & 94.66 & 51.8681 & 18.26315 \\
ap_vb_t2 & 33 & 14.43 & 104.20 & 59.9105 & 20.64999 \\
ap_vb_t3 & 33 & 15.67 & 88.03 & 57.4018 & 17.16410 \\
ap_vb_t4 & 33 & 6.07 & 68.96 & 40.6397 & 16.04717 \\
ap_vb_t5 & 33 & 27.12 & 86.07 & 57.3423 & 15.81570 \\
ap_vb_t6 & 33 & 13.36 & 98.16 & 59.0014 & 20.80572 \\
ap_tb_t1 & 33 & 21.87 & 133.15 & 70.1027 & 20.51294 \\
ap_tb_t2 & 33 & 36.29 & 104.36 & 68.9056 & 17.10688 \\
ap_tb_t3 & 33 & 30.73 & 118.41 & 68.2558 & 19.18326 \\
ap_tb_t4 & 33 & 21.18 & 103.75 & 73.3679 & 17.78754 \\
ap_tb_t5 & 33 & 33.01 & 117.78 & 71.6329 & 16.53148 \\
ap_tb_t6 & 33 & 26.22 & 94.81 & 68.1040 & 17.76235 \\
ap_dr_t1 & 33 & 27.92 & 115.35 & 66.3127 & 18.76133 \\
ap_dr_t2 & 33 & 51.55 & 100.72 & 70.6681 & 10.59812 \\
ap_dr_t3 & 33 & 40.86 & 103.63 & 65.2630 & 12.39701 \\
ap_dr_t4 & 33 & 37.58 & 89.93 & 65.7167 & 13.01747 \\
ap_dr_t5 & 33 & 10.38 & 131.79 & 75.0690 & 18.92732 \\
ap_dr_t6 & 33 & 29.61 & 109.59 & 67.9621 & 18.04126
\end{tabular}

Table N6 Mean Rates of Perceived Exertion per Trial for Volleyball, Tae-Bo and DDR

\begin{tabular}{cccccc}
\hline $\begin{array}{c}\text { Trial for } \\
\text { Activity Type }\end{array}$ & $\mathrm{N}$ & Minimum & Maximum & Mean & Std. Deviation \\
\hline rpe_vb_t1 & 33 & .00 & 10.00 & 2.6061 & 2.57317 \\
rpe_vb_t2 & 33 & .00 & 10.00 & 2.9394 & 3.08159 \\
rpe_vb_t3 & 33 & .00 & 9.00 & 2.2727 & 2.78694 \\
rpe_vb_t4 & 33 & .00 & 8.00 & 1.6970 & 2.41719 \\
rpe_vb_t5 & 33 & .00 & 8.00 & 2.3030 & 2.44291 \\
rpe_vb_t6 & 33 & .00 & 10.00 & 3.1515 & 2.88445 \\
rpe_tb_t1 & 33 & .00 & 10.00 & 4.4545 & 3.58025 \\
rpe_tb_t2 & 33 & .00 & 10.00 & 4.6061 & 2.73792 \\
rpe_tb_t3 & 33 & .00 & 10.00 & 4.9697 & 3.49540 \\
rpe_tb_t4 & 33 & .00 & 10.00 & 5.0606 & 3.69069 \\
rpe_tb_t5 & 33 & .00 & 10.00 & 3.3030 & 3.39563 \\
rpe_tb_t6 & 33 & .00 & 10.00 & 4.3636 & 3.65563 \\
rpe_dr_t1 & 33 & .00 & 10.00 & 2.7273 & 2.77571 \\
rpe_dr_t2 & 33 & .00 & 10.00 & 3.5455 & 3.39200 \\
rpe_dr_t3 & 33 & .00 & 10.00 & 3.0303 & 3.11734 \\
rpe_dr_t4 & 33 & .00 & 10.00 & 3.5455 & 3.11339 \\
rpe_dr_t5 & 33 & .00 & 10.00 & 3.1212 & 3.51566 \\
rpe_dr_t6 & 33 & .00 & 10.00 & 3.6061 & 3.24942
\end{tabular}


Table N7 Mean Enjoyment Scores per Trial for Volleyball, Tae-Bo and DDR

\begin{tabular}{cccccc}
\hline $\begin{array}{c}\text { Trial for } \\
\text { Activity Type }\end{array}$ & $\mathrm{N}$ & Minimum & Maximum & Mean & Std. Deviation \\
\hline enj_vb_t1 & 33 & 12.00 & 35.00 & 29.6667 & 6.99405 \\
enj_vb_t2 & 33 & 14.00 & 35.00 & 30.1515 & 6.91069 \\
enj_vb_t3 & 33 & 10.00 & 35.00 & 30.3636 & 7.36044 \\
enj_vb_t4 & 33 & 7.00 & 35.00 & 29.7273 & 7.39203 \\
enj_vb_t5 & 33 & 14.00 & 35.00 & 30.5758 & 6.69902 \\
enj_vb_t6 & 33 & 9.00 & 35.00 & 29.5455 & 7.08912 \\
enj_tb_t1 & 33 & 7.00 & 35.00 & 25.8485 & 8.89916 \\
enj_tb_t2 & 33 & 7.00 & 35.00 & 23.6970 & 9.53770 \\
enj_tb_t3 & 33 & 7.00 & 35.00 & 20.0606 & 11.01119 \\
enj_tb_t4 & 33 & 7.00 & 35.00 & 21.3030 & 10.53709 \\
enj_tb_t5 & 33 & 7.00 & 35.00 & 22.5455 & 10.64510 \\
enj_tb_t6 & 33 & 7.00 & 35.00 & 21.2727 & 11.25909 \\
enj_dr_t1 & 33 & 15.00 & 35.00 & 32.0303 & 5.32966 \\
enj_dr_t2 & 33 & 10.00 & 35.00 & 31.7879 & 6.26876 \\
enj_dr_t3 & 33 & 9.00 & 35.00 & 31.5152 & 6.73202 \\
enj_dr_t4 & 33 & 10.00 & 35.00 & 31.7576 & 6.59560 \\
enj_dr_t5 & 33 & 14.00 & 35.00 & 31.1818 & 6.93476 \\
enj_dr_t6 & 33 & 13.00 & 35.00 & 31.6061 & 6.60937
\end{tabular}

Table N8 Collapsed Means for Heart Rate, RPE, Enjoyment, Step Count, Active Time, and Total Elapsed Time for Volleyball, Tae-Bo and DDR

\begin{tabular}{cccccc}
\hline Variable & $\mathrm{N}$ & Minimum & Maximum & Mean & Std. Deviation \\
\hline hr_vb_av & 33 & 99.83 & 147.17 & 121.2677 & 12.01286 \\
hr_tb_av & 33 & 85.83 & 143.00 & 125.5859 & 13.11594 \\
hr_dr_av & 33 & 99.83 & 166.50 & 128.8333 & 13.23099 \\
rpe_vb_av & 33 & .00 & 8.17 & 2.4949 & 2.35204 \\
rpe_tb_av & 33 & .33 & 10.00 & 4.4596 & 2.91489 \\
rpe_dr_av & 33 & .00 & 10.00 & 3.2626 & 2.58099 \\
enj_vb_av & 33 & 14.00 & 35.00 & 30.0051 & 6.03570 \\
enj_tb_av & 33 & 7.33 & 35.00 & 22.4545 & 8.69090 \\
enj_dr_av & 33 & 12.50 & 35.00 & 31.6465 & 5.95363 \\
stp_vb_av & 33 & 515.67 & 1718.33 & 1104.1818 & 313.31732 \\
stp_tb_av & 33 & 580.33 & 1941.50 & 1262.5101 & 343.40634 \\
stp_dr_av & 33 & 782.17 & 2087.67 & 1490.7121 & 322.30238 \\
at_vb_av & 33 & 457.50 & 1244.33 & 848.5303 & 195.40118 \\
at_tb_av & 33 & 549.17 & 1179.50 & 904.0707 & 163.71955 \\
at_dr_av & 33 & 672.67 & 1319.83 & 1004.6414 & 151.11426 \\
tt_vb_av & 33 & 1418.17 & 1679.33 & 1577.1061 & 69.20068 \\
tt_tb_av & 33 & 1131.50 & 1398.33 & 1293.5152 & 57.32472 \\
tt_dr_av & 33 & 1290.83 & 1575.50 & 1475.8636 & 54.20906
\end{tabular}




\section{Appendix $\mathrm{O}$}

Mean Scores According to Group (Gender, Grade Level, BMI)

Table O1 Heart Rate Means According to Gender

\begin{tabular}{ccccc}
\hline Gender & & $\mathrm{hr} \_\mathrm{vb}$ av & $\mathrm{hr}$ tb_av & $\mathrm{hr}$ dr_av \\
\hline Female & Mean & 119.5000 & 128.1923 & 133.8846 \\
& $\mathrm{~N}$ & 13 & 13 & 13 \\
& Std. Deviation & 7.28742 & 10.27624 & 13.30594 \\
Male & Mean & 122.4167 & 123.8917 & 125.5500 \\
& $\mathrm{~N}$ & 20 & 20 & 20 \\
& Std. Deviation & 14.35194 & 14.67536 & 12.41816 \\
Total & Mean & 121.2677 & 125.5859 & 128.8333 \\
& $\mathrm{~N}$ & 33 & 33 & 33 \\
& Std. Deviation & 12.01286 & 13.11594 & 13.23099
\end{tabular}

Table O2 Heart Rate Means According to Grade Level

\begin{tabular}{ccccc}
\hline Grade Level & & hr_vb_av & hr tb_av & hr_dr_av \\
\hline Grade 5 & Mean & 122.7143 & 127.8889 & 129.9683 \\
& $\mathrm{~N}$ & 21 & 21 & 21 \\
Grade 6 & Std. Deviation & 12.98473 & 9.05007 & 10.62252 \\
& Mean & 118.7361 & 121.5556 & 126.8472 \\
Total & $\mathrm{N}$ & 12 & 12 & 12 \\
& Std. Deviation & 10.11286 & 17.99121 & 17.24357 \\
& Mean & 121.2677 & 125.5859 & 128.8333 \\
& N & 33 & 33 & 33 \\
& Std. Deviation & 12.01286 & 13.11594 & 13.23099
\end{tabular}

Table O3 Heart Rate Means According to Body Mass Index

\begin{tabular}{ccccc}
\hline $\begin{array}{c}\text { Body Mass } \\
\text { Index }\end{array}$ & & hr_vb_av & hr_tb_av & hr_dr_av \\
\hline $\begin{array}{c}\text { Healthy } \\
\text { Weight } \\
\left(<85^{\text {th }}\right.\end{array}$ & & & & \\
Percentile $)$ & Mean & 117.2917 & 124.1167 & 124.5417 \\
& $\mathrm{~N}$ & 20 & 20 & 20 \\
Overweight & Std. Deviation & 11.16763 & 14.29095 & 11.29183 \\
$\left(\geq 85^{\text {th }}\right.$ & & & & \\
Percentile $)$ & Mean & 127.3846 & 127.8462 & 135.4359 \\
& $\mathrm{~N}$ & 13 & 13 & 13 \\
Total & Std. Deviation & 10.97606 & 11.23579 & 13.67539 \\
& Mean & 121.2677 & 125.5859 & 128.8333 \\
& $\mathrm{~N}$ & 33 & 33 & 33 \\
& Std. Deviation & 12.01286 & 13.11594 & 13.23099
\end{tabular}


Table O4 Step Count Means According to Gender

\begin{tabular}{lcccc}
\hline Gender & & stp_vb_av & stp_tb_av & stp_dr_av \\
\hline Female & Mean & 1000.9103 & 1289.3846 & 1548.1282 \\
& $\mathrm{~N}$ & 13 & 13 & 13 \\
Male & Std. Deviation & 260.18749 & 372.79336 & 307.28547 \\
& Mean & 1171.3083 & 1245.0417 & 1453.3917 \\
& $\mathrm{~N}$ & 20 & 20 & 20 \\
Total & Std. Deviation & 332.47341 & 331.70236 & 334.05939 \\
& Mean & 1104.1818 & 1262.5101 & 1490.7121 \\
& $\mathrm{~N}$ & 33 & 33 & 33 \\
& Std. Deviation & 313.31732 & 343.40634 & 322.30238
\end{tabular}

Table O5 Step Count Means According to Grade Level

\begin{tabular}{ccccc}
\hline Grade & & stp_vb_av & stp_tb_av & stp_dr_av \\
\hline Grade 5 & Mean & 1084.5317 & 1204.0556 & 1465.0476 \\
& $\mathrm{~N}$ & 21 & 21 & 21 \\
Grade 6 & Std. Deviation & 317.24246 & 361.67188 & 359.96139 \\
& Mean & 1138.5694 & 1364.8056 & 1535.6250 \\
& $\mathrm{~N}$ & 12 & 12 & 12 \\
Total & Std. Deviation & 317.11962 & 295.45566 & 251.29489 \\
& Mean & 1104.1818 & 1262.5101 & 1490.7121 \\
& $\mathrm{~N}$ & 33 & 33 & 33 \\
& Std. Deviation & 313.31732 & 343.40634 & 322.30238
\end{tabular}

Table O6 Step Count Means According to Body Mass Index

\begin{tabular}{ccccc}
\hline $\begin{array}{c}\text { Body Mass } \\
\text { Index }\end{array}$ & & & & \\
\hline Healthy & stp_vb_av & stp_tb_av & stp_dr_av \\
Weight & Mean & 1098.9333 & 1285.1417 & 1504.3083 \\
& $\mathrm{~N}$ & 20 & 20 & 20 \\
Overweight & Std. Deviation & 335.09492 & 334.71634 & 312.25219 \\
& Mean & 1112.2564 & 1227.6923 & 1469.7949 \\
& $\mathrm{~N}$ & 13 & 13 & 13 \\
Total & Std. Deviation & 289.60974 & 367.31281 & 349.07140 \\
& Mean & 1104.1818 & 1262.5101 & 1490.7121 \\
& $\mathrm{~N}$ & 33 & 33 & 33 \\
& Std. Deviation & 313.31732 & 343.40634 & 322.30238
\end{tabular}


Table O7 Mean Percent Active Time According to Gender

\begin{tabular}{ccccc}
\hline Gender & & ap_dr_av & ap_tb_av & ap_vb_av \\
\hline Female & Mean & 68.6106 & 72.2636 & 51.1687 \\
& $\mathrm{~N}$ & 13 & 13 & 13 \\
& Std. Deviation & 7.08856 & 13.16574 & 12.91495 \\
Male & Mean & 68.4258 & 68.6301 & 56.4353 \\
& $\mathrm{~N}$ & 20 & 20 & 20 \\
Total & Std. Deviation & 11.69055 & 12.37820 & 13.01094 \\
& Mean & 68.4986 & 70.0615 & 54.3606 \\
& $\mathrm{~N}$ & 33 & 33 & 33 \\
& Std. Deviation & 9.99992 & 12.61848 & 13.03420
\end{tabular}

Table O8 Mean Percent Active Time According to Grade Level

\begin{tabular}{ccccc}
\hline Grade Level & & ap_dr_av & ap_tb_av & ap_vb_av \\
\hline Grade 5 & Mean & 67.5382 & 66.8533 & 51.3270 \\
& $\mathrm{~N}$ & 21 & 21 & 21 \\
Grade 6 & Std. Deviation & 10.81878 & 11.63609 & 11.10612 \\
& Mean & 70.1793 & 75.6758 & 59.6694 \\
Total & $\mathrm{N}$ & 12 & 12 & 12 \\
& Std. Deviation & 8.55870 & 12.76667 & 14.88781 \\
& Mean & 68.4986 & 70.0615 & 54.3606 \\
& N & 33 & 33 & 33 \\
& Std. Deviation & 9.99992 & 12.61848 & 13.03420
\end{tabular}

Table O9 Mean Percent Active Time According to Body Mass Index

\begin{tabular}{|c|c|c|c|c|}
\hline BMI & & ap dr av & ap tb av & $a p$ vb av \\
\hline \multirow[t]{3}{*}{$\begin{array}{l}\text { Healthy } \\
\text { Weight }\end{array}$} & Mean & 68.3132 & 70.8167 & 53.6644 \\
\hline & $\mathrm{N}$ & 20 & 20 & 20 \\
\hline & Std. Deviation & 10.24626 & 12.10704 & 13.28053 \\
\hline \multirow[t]{3}{*}{ Overweight } & Mean & 68.7839 & 68.8996 & 55.4316 \\
\hline & $\mathrm{N}$ & 13 & 13 & 13 \\
\hline & Std. Deviation & 10.01446 & 13.78780 & 13.10475 \\
\hline \multirow[t]{3}{*}{ Total } & Mean & 68.4986 & 70.0615 & 54.3606 \\
\hline & $\mathrm{N}$ & 33 & 33 & 33 \\
\hline & Std. Deviation & 9.99992 & 12.61848 & 13.03420 \\
\hline
\end{tabular}


Table O10 Mean Rate of Perceived Exertion According to Grade Level

\begin{tabular}{ccccc}
\hline Grade & & rpe $v$ vb_av & rpe tb_av & rpe_dr_av \\
\hline Grade 5 & Mean & 2.2222 & 4.0317 & 2.8095 \\
& $\mathrm{~N}$ & 21 & 21 & 21 \\
Grade 6 & Std. Deviation & 2.03943 & 2.91672 & 2.18917 \\
& Mean & 2.9722 & 5.2083 & 4.0556 \\
& $\mathrm{~N}$ & 12 & 12 & 12 \\
Total & Std. Deviation & 2.85317 & 2.87898 & 3.09638 \\
& Mean & 2.4949 & 4.4596 & 3.2626 \\
& $\mathrm{~N}$ & 33 & 33 & 33 \\
& Std. Deviation & 2.35204 & 2.91489 & 2.58099
\end{tabular}

Table O11 Mean Rate of Perceived Exertion According to Gender

\begin{tabular}{ccccc}
\hline Gender & & rpe_vb_av & rpe tb_av & rpe_dr_av \\
\hline Female & Mean & 2.5000 & 4.7051 & 3.6538 \\
& $\mathrm{~N}$ & 13 & 13 & 13 \\
Male & Std. Deviation & 2.72421 & 2.74381 & 2.82717 \\
& Mean & 2.4917 & 4.3000 & 3.0083 \\
& $\mathrm{~N}$ & 20 & 20 & 20 \\
Total & Std. Deviation & 2.15175 & 3.08012 & 2.44918 \\
& Mean & 2.4949 & 4.4596 & 3.2626 \\
& $\mathrm{~N}$ & 33 & 33 & 33 \\
& Std. Deviation & 2.35204 & 2.91489 & 2.58099
\end{tabular}

Table O12 Mean Rate of Perceived Exertion According to Body Mass Index

\begin{tabular}{ccccc}
\hline $\begin{array}{c}\text { Body Mass } \\
\text { Index }\end{array}$ & & rpe_vb_av & rpe_tb_av & rpe_dr_av \\
\hline Healthy & & & & \\
Weight & Mean & 2.6417 & 4.3917 & 3.2083 \\
& $\mathrm{~N}$ & 20 & 20 & 20 \\
& Std. Deviation & 2.29114 & 2.69843 & 2.07172 \\
Overweight & Mean & 2.2692 & 4.5641 & 3.3462 \\
& $\mathrm{~N}$ & 13 & 13 & 13 \\
Total & Std. Deviation & 2.51986 & 3.33301 & 3.30996 \\
& Mean & 2.4949 & 4.4596 & 3.2626 \\
& $\mathrm{~N}$ & 33 & 33 & 33 \\
& Std. Deviation & 2.35204 & 2.91489 & 2.58099
\end{tabular}


Table O13 Mean Enjoyment Score According to Grade Level

\begin{tabular}{ccccc}
\hline Grade & & enj_vb_av & enj_tb_av & enj_dr_av \\
\hline Grade 5 & Mean & 29.2698 & 23.3175 & 30.4603 \\
& N & 21 & 21 & 21 \\
Grade 6 & Std. Deviation & 6.23843 & 9.00215 & 7.09693 \\
& Mean & 31.2917 & 20.9444 & 33.7222 \\
& N & 12 & 12 & 12 \\
Total & Std. Deviation & 5.69029 & 8.27505 & 2.03794 \\
& Mean & 30.0051 & 22.4545 & 31.6465 \\
& N & 33 & 33 & 33 \\
& Std. Deviation & 6.03570 & 8.69090 & 5.95363
\end{tabular}

Table O14 Mean Enjoyment Score According to Gender

\begin{tabular}{ccccc}
\hline Gender & & enj_vb_av & enj_tb_av & enj_dr_av \\
\hline Female & Mean & 32.0641 & 25.5513 & 33.3974 \\
& N & 13 & 13 & 13 \\
\multirow{4}{*}{ Male } & Std. Deviation & 5.37033 & 9.19626 & 3.89238 \\
& Mean & 28.6667 & 20.4417 & 30.5083 \\
& N & 20 & 20 & 20 \\
Total & Std. Deviation & 6.19304 & 7.93546 & 6.83140 \\
& Mean & 30.0051 & 22.4545 & 31.6465 \\
& N & 33 & 33 & 33 \\
& Std. Deviation & 6.03570 & 8.69090 & 5.95363
\end{tabular}

Table O15 Mean Enjoyment Score According to Body Mass Index

\begin{tabular}{ccccc}
\hline $\begin{array}{c}\text { Body Mass } \\
\text { Index }\end{array}$ & & enj_vb_av & enj_tb_av & enj_dr_av \\
\hline Healthy & & & & \\
Weight & Mean & 30.0750 & 24.1667 & 32.0500 \\
& $\mathrm{~N}$ & 20 & 20 & 20 \\
& Std. Deviation & 5.78627 & 9.21399 & 5.29512 \\
Overweight & Mean & 29.8974 & 19.8205 & 31.0256 \\
& $\mathrm{~N}$ & 13 & 13 & 13 \\
Total & Std. Deviation & 6.64181 & 7.38882 & 7.03129 \\
& Mean & 30.0051 & 22.4545 & 31.6465 \\
& $\mathrm{~N}$ & 33 & 33 & 33 \\
& Std. Deviation & 6.03570 & 8.69090 & 5.95363
\end{tabular}




\section{Appendix P}

Pearson Product-Moment Correlation Coefficients Among Dependent Variables Table P1 Heart Rate and Rate of Perceived Exertion

$\begin{array}{ccccc}\text { Pair } & \text { hr_vb_av \& rpe_vb_av } & \text { N } & \text { Correlation } & \text { Sig. } \\ 1 & 33 & .048 & .791 \\ \text { Pair } & \text { hr_tb_av \& rpe_tb_av } & 33 & -.072 & .691 \\ 2 & & & & \\ \text { Pair } & \text { hr_dr_av \& rpe_dr_av } & 33 & .026 & .884 \\ 3 & & & \end{array}$

Table P2 Enjoyment and Rate of Perceived Exertion

$\begin{array}{ccccc}\text { Pair } & \text { rpe_vb_av \& enj_vb_av } & \text { N } & \text { Correlation } & \text { Sig. } \\ 1 & 33 & -.051 & .780 \\ \text { Pair } & \text { rpe_tb_av \& enj_tb_av } & 33 & -.302 & .087 \\ 2 & & & & \\ \text { Pair } & \text { rpe_dr_av \& enj_dr_av } & 33 & .069 & .703 \\ 3 & & & \end{array}$

Table P3 Step Count and Active Time

$\begin{array}{ccccc}\text { Pair } & \text { stp_vb_av \& at_vb_av } & \text { N } & \text { Correlation } & \text { Sig. } \\ 1 & & 33 & .978 & .000 \\ \text { Pair } & \text { stp_tb_av \& at_tb_av } & 33 & .935 & .000 \\ 2 & & & & .833 \\ \text { Pair } & \text { stp_dr_av \& at_dr_av } & 33 & & \end{array}$

Table P4 Enjoyment and Step Count

$\begin{array}{llccc}\text { Pair } & \text { enj_vb_av \& stp_vb_av } & \text { N } & \text { Correlation } & \text { Sig. } \\ 1 & & .150 & .404 \\ \text { Pair } & \text { enj_tb_av \& stp_tb_av } & 33 & .019 & .918 \\ 2 & & & & \\ \text { Pair } & \text { enj_dr_av \& stp_dr_av } & 33 & .284 & .109 \\ 3 & & & \end{array}$


Table P5 Active Time and Elapsed Time

$\begin{array}{llccc}\text { Pair } & \text { at_vb_av \& tt_vb_av } & \mathrm{N} & \text { Correlation } & \text { Sig. } \\ \begin{array}{l}\text { 1 } \\ \text { Pair }\end{array} & \text { at_tb_av \& tt_tb_av } & 33 & -.167 & .352 \\ 2 & & 33 & .173 & .336 \\ \text { Pair } & \text { at_dr_av \& tt_dr_av } & 33 & .189 & .293 \\ 3 & & & \end{array}$

Table P6 Heart Rate and Step Count

$\begin{array}{llccc}\text { Pair } & \text { hr_vb_av \& stp_vb_av } & \text { N } & \text { Correlation } & \text { Sig. } \\ \begin{array}{l}1 \\ \text { Pair }\end{array} & \text { hr_tb_av \& stp_tb_av } & 33 & .254 & .153 \\ 2 & & 33 & .083 & .645 \\ \text { Pair } & \text { hr_dr_av \& stp_dr_av } & 33 & .124 & .492 \\ 3 & & & \end{array}$

Table P7 Heart Rate and Enjoyment

$\begin{array}{llccc}\text { Pair } & \text { hr_vb_av \& enj_vb_av } & \text { N } & \text { Correlation } & \text { Sig. } \\ \begin{array}{c}1 \\ \text { Pair }\end{array} & \text { hr_tb_av \& enj_tb_av } & 33 & .023 & .900 \\ 2 & & 33 & -.145 & .421 \\ \text { Pair } & \text { hr_dr_av \& enj_dr_av } & 33 & .209 & .244 \\ 3 & & & \end{array}$

Table P8 Heart Rate and Elapsed Time

\begin{tabular}{|c|c|c|c|c|}
\hline & hr_vb_av \& tt_vb_av & 33 & .279 & .115 \\
\hline $\begin{array}{l}\text { Pair } \\
2\end{array}$ & hr_tb_av \& tt_tb_av & 33 & -.104 & .565 \\
\hline $\begin{array}{l}\text { Pair } \\
3\end{array}$ & hr_dr_av \& tt_dr_av & 33 & -.306 & .083 \\
\hline
\end{tabular}


Table P9 Heart Rate and Percent Active Time

\begin{tabular}{|c|c|c|c|c|}
\hline & & $\mathrm{N}$ & Correlation & Sig. \\
\hline $\begin{array}{l}\text { Pair } \\
1\end{array}$ & hr_vb_av \& Rel_vb_AT & 33 & .187 & .297 \\
\hline $\begin{array}{l}\text { Pair } \\
2\end{array}$ & hr_tb_av \& Rel_tb_AT & 33 & .140 & .437 \\
\hline $\begin{array}{l}\text { Pair } \\
3\end{array}$ & hr_dr_av \& Rel_dr_AT & 33 & .030 & .869 \\
\hline
\end{tabular}

Table P10 Enjoyment and Percent Active Time

$\begin{array}{ccccc}\text { Pair } & \text { enj_vb_av \& Rel_vb_AT } & \text { N } & \text { Correlation } & \text { Sig. } \\ \begin{array}{c}\text { 1 } \\ \text { Pair }\end{array} & \text { enj_tb_av \& Rel_tb_AT } & 33 & .248 & .165 \\ 2 & & 33 & -.024 & .896 \\ \text { Pair } & \text { enj_dr_av \& Rel_dr_AT } & 33 & .183 & .308 \\ 3 & & & \end{array}$

Table P11 Enjoyment and Elapsed Time

\begin{tabular}{|c|c|c|c|c|}
\hline & & $\mathrm{N}$ & Correlation & Sig. \\
\hline $\begin{array}{c}\text { Pair } \\
1\end{array}$ & enj_vb_av \& tt_vb_av & 33 & -.263 & .140 \\
\hline $\begin{array}{c}\text { Pair } \\
2\end{array}$ & enj_tb_av \& $t$ t $\_t b \_a v$ & 33 & -.135 & 45 \\
\hline $\begin{array}{c}\text { Pair } \\
3\end{array}$ & enj_dr_av \& tt_dr_av & 33 & -.345 & .04 \\
\hline
\end{tabular}

